Aus der Abteilung Psychiatrie und Psychotherapie

(Prof. Dr. med. P. Falkai)

im Zentrum Psychosoziale Medizin

der Medizinischen Fakultät der Universität Göttingen

\title{
Evaluierung eines Qualitätssicherungsprogramms im interdisziplinären Fachgebiet am Beispiel der Schlafmedizin
}

\author{
INAUGURAL-DISSERTATION \\ zur Erlangung des Doktorgrades \\ der Medizinischen Fakultät \\ der Georg-August-Universität zu Göttingen
}

vorgelegt von

Ludmila Utte

aus

Michailowka/Kasachstan

Göttingen 2012 
Dekan:

1. Berichterstatterin:

2. Berichterstatter:

3. Berichterstatter:

Tag der mündlichen Prüfung:
Prof. Dr. med. M. P. Schön

Prof. Dr. rer. nat. A. Rodenbeck

Prof. Dr. med. S. Andreas

Prof. Dr. rer. nat. P. Virsik-Köpp 


\section{Inhaltsverzeichnis}

Abbildungsverzeichnis

Tabellenverzeichnis

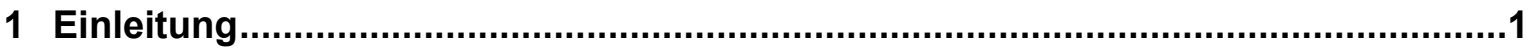

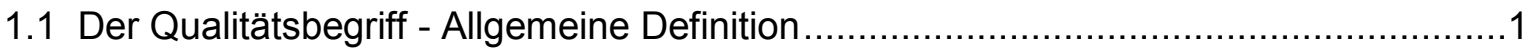

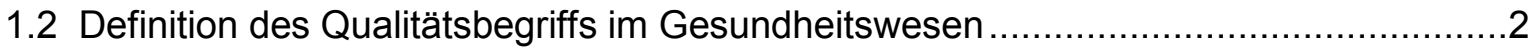

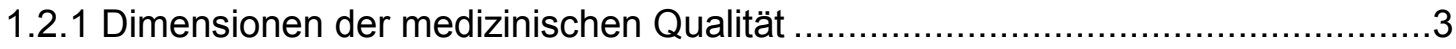

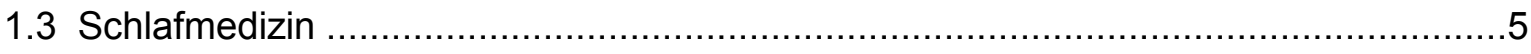

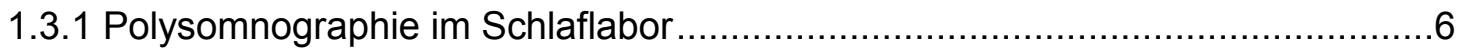

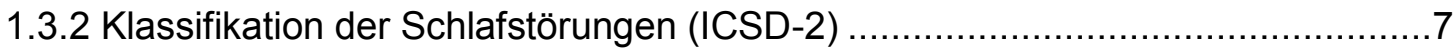

1.4 Qualitätssicherung und förderung im Gesundheitswesen .................................. 8

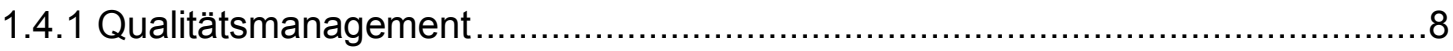

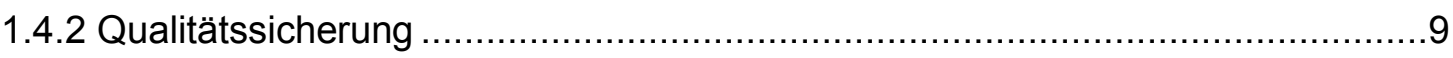

1.4.3 Richt- und Leitlinien als qualitätssichernde Maßnahme im Gesundheitswesen 10

1.4.4 Verfahren zur Bewertung von Qualität im Gesundheitswesen .......................11

1.4.5 Entwicklung von Qualitätsindikatoren zum Messen von Versorgungsqualität...12

1.4.5.1 Entwicklung der Qualitätsindikatoren für den Fachbereich Schlafmedizin 13

1.5 Qualitätsbezogene Aktivitäten der DGSM in der deutschen Schlafmedizin ...............14

1.5.1 Bisherige Verfahren zur Überprüfung der Versorgungsqualität ......................14

1.5.2 Aktuelle qualitätsbezogene Aktivitäten der DGSM: Verfahrensänderung .........15

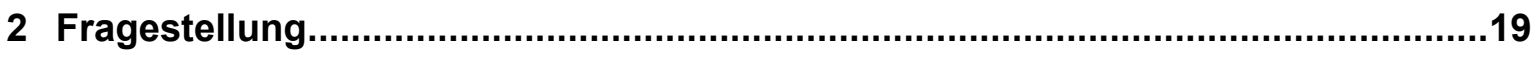

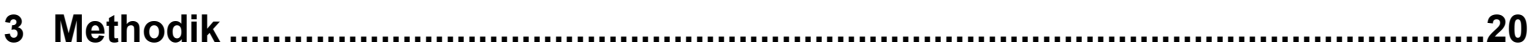

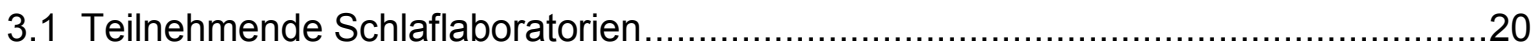

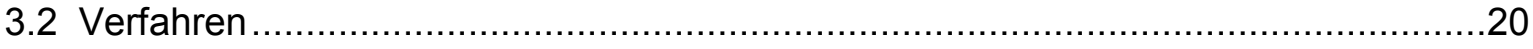

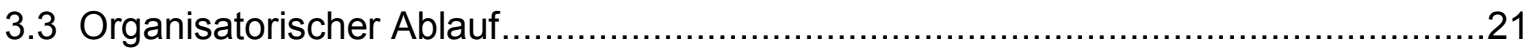

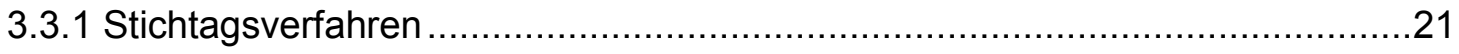

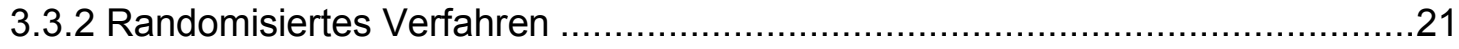

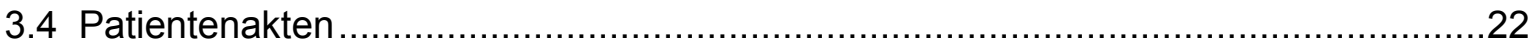

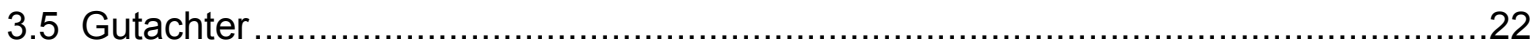


3.6 Die Checkliste qualitätsrelevanter Prozessmerkmale und das Manual mit den Beurteilungskriterien

3.7 Entscheidungskriterien für die Revisitation und das Fortbestehen der Akkreditierung .27

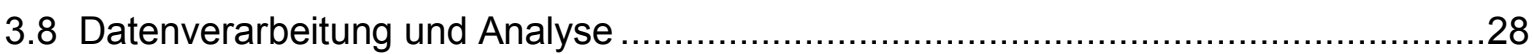

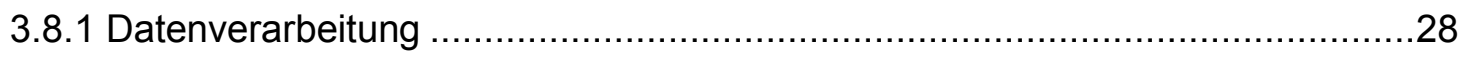

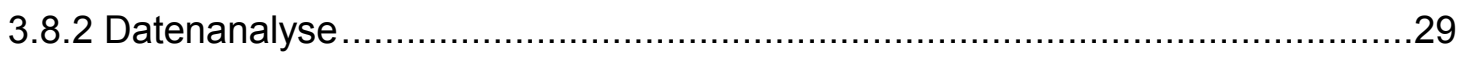

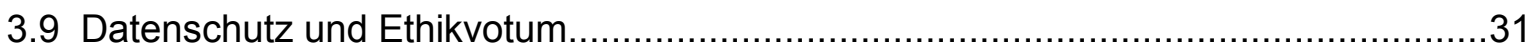

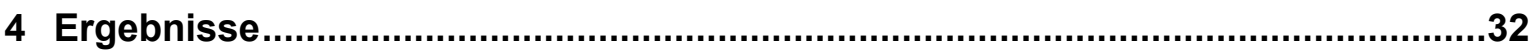

4.1 Ergebnisse zum organisatorischen und zeitlichen Verlauf...................................32

4.2 Ergebnis der Weiterakkreditierung und die Abhängigkeit des jeweiligen Verfahrens.33

4.3 Ergebnis der Revisitationsempfehlungen der Gutachter........................................34

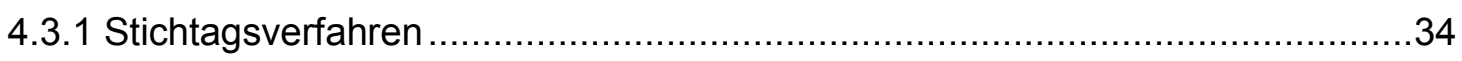

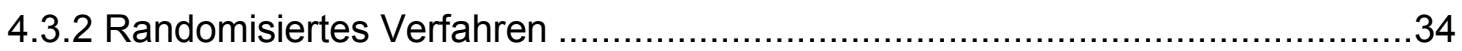

4.4 Verhalten der Gutachter bezüglich der Revisitationsentscheidung im Vergleich der beiden methodischen Verfahren ................................................................ 35

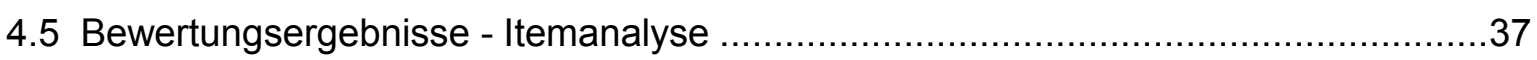

4.5.1 Schlaflaboratorien mit Weiterakkreditierung - verfahrensbezogen...................37

4.5.2 Schlaflaboratorien ohne Weiterakkreditierung - nicht verfahrensbezogen ........43

4.5.3 Ergebnis der visuellen Analogskala .........................................................44

4.6 Untersuchung der Items hinsichtlich ihrer Einflussnahme auf die Entscheidung des Gutachters zur Revisitation ..........................................................................46

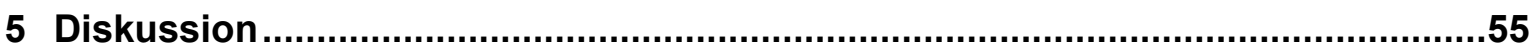

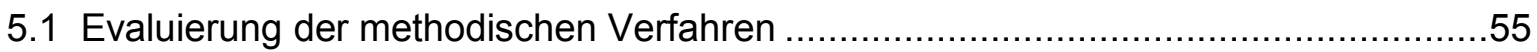

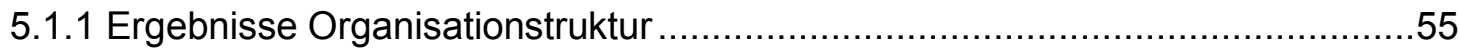

5.1.2 Ergebnisse Weiterakkreditierung und Revisitationsentscheidung...................56

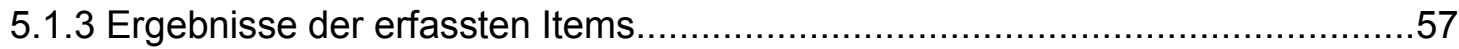

5.1.4 Empfehlung eines Verfahrens zur weiteren Durchführung im Rahmen des Qualitätssicherungsprogramms der DGSM ............................................. 58

5.2 Die Qualität in den DGSM-akkreditierten Schlaflaboratorien .................................59

5.3 Abhängigkeit der Items in Bezug auf die Gutachterempfehlung zur Revisitation .......65

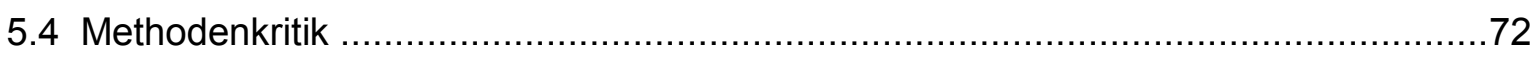

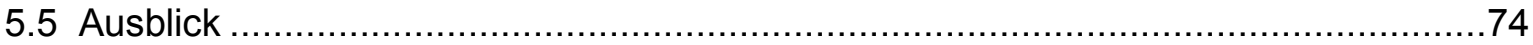




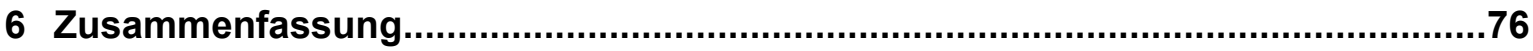

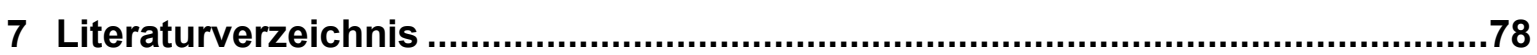

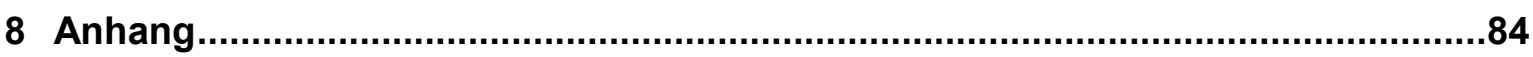

8.1 Checkliste inklusive Beurteilungsmanual zur Qualitätssicherung in Schlaflaboratorien

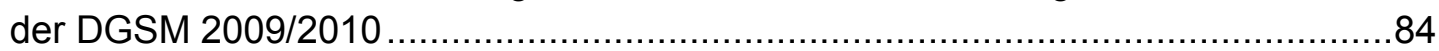

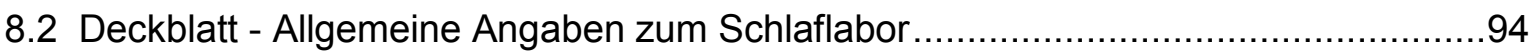

8.3 Merkblatt zum Qualitätssicherungsverfahren der DGSM .....................................

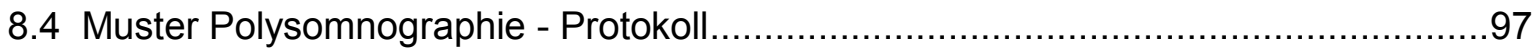

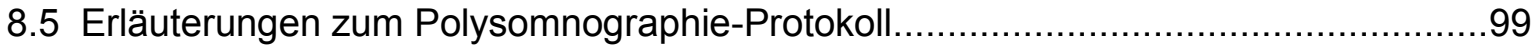

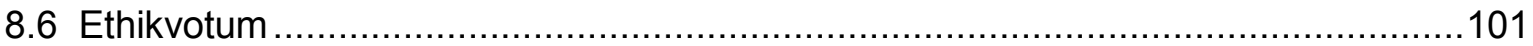

8.7 Erfassung der bewerteten Checkliste für die Schlaflaboratorien mit Weiterakkreditierung (verfahrensbezogen)........................................................ 102

8.8 Erfassung der bewerteten Checkliste für die Schlaflaboratorien ohne Weiterakkreditierung (nicht verfahrensbezogen). 105 


\section{Abbildungsverzeichnis}

Abbildung 1. Kardiorespiratorische Polysomnographie (PSG) im Schlaflabor .7

Abbildung 2. Zusammenhang des „Plan-Do-Check-Act (PDCA) Zyklus“ und des PeerReview-Verfahrens der DGSM (aus Kutschmann et al. 2002, S. 91) ....18

Abbildung 3. Beurteilungsfeld Diagnosesicherung der Checkliste qualitäts-relevanter Prozessmerkmale im DGSM-Peer-Review-Verfahren 2009/2010 . .24

Abbildung 4. Beurteilungsfeld Anamnese der Checkliste qualitätsrelevanter Prozessmerkmale im DGSM-Peer-Review-Verfahren 2009/2010 . .25

Abbildung 5. Visuelle Analogskala zur Beurteilung des Gesamtprozesses bezüglich der Entsprechung des aktuellen Standes wissenschaftlicher Erkenntnisse, Empfehlungen und Leitlinien.

Abbildung 6. Übersicht des Kodierschemas im Tabellenkalkulationsprogramm Excel .28

Abbildung 7. Ergebnis der Weiterakkreditierung der am Qualitätssicherungs-programm teilnehmenden Schlaflaboratorien im Vergleich des Stichtags- bzw. Randomisierten Verfahrens .33

Abbildung 8. Anteile der Schlaflaboratorien mit keiner/einer/mehr als zwei Revisitationsempfehlungen im Vergleich zwischen Stichtagsverfahren und Randomisiertem Verfahren .35

Abbildung 9. Häufigkeitsverteilung der erfassten Prozessmerkmale aus dem Beurteilungsfeld Anamnese für die weiterhin akkreditierten Schlaflabore38

Abbildung 10. Häufigkeitsverteilung der durch die Gutachter erfassten Items im Beurteilungsfeld Diagnostik in den weiterhin akkreditierten Schlaflaboratorien. 39

Abbildung 11. Häufigkeitsverteilung der erfassten Items aus dem Beurteilungsfeld Therapie für die weiterhin akkreditierten Schlaflaboratorien .41

Abbildung 12. Häufigkeitsverteilung der erfassten Items aus dem Beurteilungsfeld Entlassungsbericht für die weiterhin akkreditierten Schlaflaboratorien. ...43 Abbildung 13. Beurteilung des Gesamtprozesses durch die Gutachter mittels visueller Analogskala. .45 


\section{Tabellenverzeichnis}

Tabelle 1. Hauptgruppen der Schlafstörungen gemäß der American Academy of Sleep Medicine (AASM 2005)

Tabelle 2. Am Qualitätssicherungsverfahren teilnehmende Schlaflaboratorien mit ihrem Schwerpunkt im Fachbereich .20

Tabelle 3. Definierte Antwortmöglichkeiten zur Beurteilung der Prozessmerkmale......23

Tabelle 4. Überblick über die Kriterien zur Revisitationsentscheidung und die Fort-

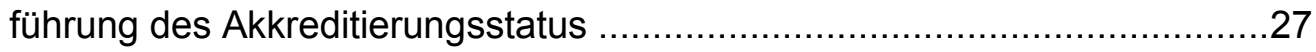

Tabelle 5. Wichtige Daten zum Ablauf des Stichtagsverfahrens und des Randomisierten Verfahrens im Vergleich

Tabelle 6. Vergleich des Stichtagsverfahrens und des Randomisierten Verfahrens im Hinblick auf die Weiterakkreditierung (Chi ${ }^{2}$-Test).

Tabelle 7. Übersicht über die Häufigkeit der Revisitationsempfehlungen der 25 an beiden Verfahren teilnehmenden Gutachter .36

Tabelle 8. Häufigkeitsverteilung der erfassten Messgrößen in den Patientenakten der weiterhin akkreditierten Schlafzentren

Tabelle 9. Häufigkeitsverteilung der Bewertung der „Zusammenfassenden Beurteilung“ der „Schlaf-, Atmungsparameter und Beinbewegungen“ im Entlassungsbericht der weiterhin akkreditierten Schlafzentren

Tabelle 10. Untersuchung der Abhängigkeit zwischen Itembewertung und der Revisitationsentscheidung des Gutachters mit Hilfe eines Chi ${ }^{2}$-Vierfeldertests .....47

Tabelle 11. Überblick über die beurteilten Prozessmerkmale (Anamnese) und ihre Häufigkeitsverteilung im Hinblick auf die Revisitationsentscheidung des Gutachters.

Tabelle 12. Gegenüberstellung der erfassten Prozessmerkmale (Diagnostik) und ihre Häufigkeitsverteilung im Hinblick auf die Revisitationsentscheidung des Gutachters.

Tabelle 13. Vergleich der bewerteten Prozessmerkmale (Diagnosesicherung) und ihre Häufigkeitsverteilung im Hinblick auf die Revisitationsentscheidung des Gutachters.

Tabelle 14. Vergleich der bewerteten Prozessmerkmale (Therapie) und ihre Häufigkeitsverteilung im Hinblick auf die Revisitationsentscheidung des Gutachters. ...52

Tabelle 15. Gegenüberstellung der bewerteten Prozessmerkmale (Entlassungsbericht) und ihre Häufigkeitsverteilung im Hinblick auf die Revisitationsentscheidung des Gutachters. 


\section{Einleitung}

\subsection{Der Qualitätsbegriff - Allgemeine Definition}

„Es gibt so viele Definitionen der Qualität wie Leute, die sie definieren, und es besteht keine Einigkeit darüber, was Qualität ist oder sein sollte“ (Imai 1993, S. 30).

Anhand des dargestellten Zitats wird offensichtlich, dass sich schon viele Wissenschaftler mit der Suche nach einer exakten Definition des Begriffes „Qualität“ beschäftigt haben. Dennoch konnte bisher keine allgemeingültige Definition verfasst werden. Nichtsdestotrotz ist es wichtig, einen Zugang zum Begriff der Qualität zu bekommen, da er die Grundlage der folgenden Untersuchung bildet. Dazu sollen einige Definitionen aufgezeigt werden.

Das Wort Qualität stammt vom lateinischen Wort „qualitas“ ab und kann übersetzt werden als „äußere oder innere Eigenschaft" oder einfach „Beschaffenheit“ (Grimm 1984, Sp. 2308).

Die Beschäftigung mit der Definition des Begriffes Qualität geht zurück bis in die Antike (HistWbPhilos 1989, Sp.1749). Der Begriff der Qualität wird sehr lange als „subjektiv“, „nur den Sinnen zugänglich“, verstanden (Schrappe 2001, S. 266). Locke, Hauptvertreter des britischen Empirismus, und Kant im Zeitalter der Aufklärung unterscheiden schließlich zwischen „subjektiven“ und „objektiven“ Qualitäten (Schrappe 2001, S. 266). Die objektiven seien „mathematischnaturwissenschaftlich“ beschreibbar, während die subjektiven Qualitäten nur durch „psychische Prozesse“ verständlich seien (Schrappe 2001, S. 266).

Einen modernen Ansatz bei der Suche nach der Begriffsdefinition liefert das Deutsche Institut für Normung e. V. (kurz DIN), die bedeutendste nationale Normungsorganisation in der Bundesrepublik Deutschland. In der DIN EN ISO-Norm 8402:1995 ist Qualität festgelegt als: „Qualität ist die Gesamtheit der Merkmale einer Einheit bezüglich ihrer Eignung, festgelegte und vorausgesetzte Erfordernisse zu erfüllen“. Dies besagt, dass Qualität gleichzeitig mehrere Merkmale in sich vereint. Die Betonung der „festgelegten und vorausgesetzten Erfordernisse“ hebt hervor, dass diese Merkmale von einer „äußeren Institution“ vorgegeben werden müssen (Schrappe 2001, S. 266). Ähnlich formulieren es Pinter und Vitt (1996), was die obige Aussage unterstreicht. Ihnen zufolge sei Qualität „relativ“ und werde „anhand der Einhaltung der vereinbarten oder vorausgesetzten Qualitätsforderung 
gemessen“ und könne als „Erfüllungsgrad dieser Forderung entsprechend bewertet werden“ (Pinter und Vitt 1996, S. 4). Im Jahre 2000 wird die Definition von Qualität der DIN EN ISO-Norm 8402 durch die DIN EN ISO-Norm 9000:2000 ersetzt. In der Neufassung bezieht sich Qualität auf die Erfüllung der "Merkmale eines Produkts, Systems oder Prozesses“, die zuvor von „Kunden und anderen interessierten Patienten" festgelegt wurden.

\subsection{Definition des Qualitätsbegriffs im Gesundheitswesen}

Die moderne Definition von Qualität stammt ursprünglich aus der Industrie. Die Gesundheitsversorgung gehört jedoch zum Dienstleistungssektor. Daher lasse sich der aus der Industrie geprägte Qualitätsbegriff nicht direkt auf das Gesundheitswesen übertragen (Jaster 1997).

Während in der industriellen Erzeugung das Produkt in gleicher Qualität angefertigt werden könne, sei in der Medizin jeder Patient individuell und es lasse sich nur bedingt ein qualitativ vergleichbares Ergebnis erreichen (Jaster 1997).

An dieser Stelle sollen zwei Ansätze einer Definition des Begriffes Qualität im Gesundheitswesen erörtert werden.

Analog zur DIN EN ISO-Norm 8402:1995 definiert Viethen den Begriff Qualität im Gesundheitswesen folgendermaßen: „Qualität medizinischer Versorgung ist die Gesamtheit der Merkmale eines Prozesses oder eines Objektes hinsichtlich der Eignung, vorgegebene Erfordernisse im Sinne des Patienten und unter Berücksichtigung des aktuellen Kenntnisstandes der Medizin zu erfüllen“ (Viethen 1995, S. 13). Er erklärt, es sei notwendig die "Merkmale, den Prozess, das Objekt und die Erfordernisse je nach Diagnose oder Therapie" zu definieren. Das Maß an erreichter Qualität ließe sich schließlich anhand der „Erfüllung oder Nicht-Erfüllung der zuvor festgelegten Kriterien“ bewerten (Viethen 1995, S. 13).

Nach Szecsenyi (1996) definiert die Joint Commission on the Accredition of Health Care Organisations (JCAHO) die Qualität im Gesundheitswesen mit den Worten: "Qualität ist der unter Anwendung des derzeitigen Wissens vom medizinischen Versorgungssystem erreichte Grad der Wahrscheinlichkeit, für den Patienten erwünschte Therapieresultate zu erzeugen und unerwünschte Behandlungsergebnisse zu vermeiden" (Szecsenyi 1996, S. 45).

Beide Definitionen stellen den Patienten in den Mittelpunkt und beinhalten den Schutz des Patienten vor unerwünschten Nebenwirkungen. Weiterhin wird an den 
Definitionen deutlich, dass der Qualitätsbegriff im Gesundheitswesen keine zeitlose Definition darstellt. „Das derzeitige Wissen“ gibt den Maßstab für die Beurteilung der Qualität an. Da technische Fortschritte und medizinische Forschung das Wissen jedoch schnell voranschreiten lassen, kann das heute noch Fortschrittliche und damit von hoher Qualität Erscheinende, bereits morgen unzeitgemäß sein und der Qualität nicht mehr entsprechen (Viethen1995). Zusätzlich könne der Arzt als Individuum verstanden werden und sich daraus unterschiedliche Behandlungsansätze ergeben (Jaster 1997).

Hinzu kommt, dass sich „das derzeitige Wissen“ zwischen einem Haus- und einem Facharzt deutlich unterscheiden kann und daher unterschiedlich definiert werden müsste.

Die Liste an Definitionen zur Qualität in der Gesundheitsversorgung wie auch zum allgemeinen Begriff der Qualität ließe sich noch weiter fortführen, ohne dass eine definitive, allgemeingültige Begriffsbestimmung vorgenommen werden kann. Festzuhalten bleibt, dass es im Gesundheitswesen schwierig ist, Qualität zu messen. Das Erreichen definierter Behandlungsziele hängt in hohem Maß ab von dem behandelnden Arzt und dem Patienten in ihrer Individualität.

\subsubsection{Dimensionen der medizinischen Qualität}

Trotz des Fehlens einer allgemeingültigen Begriffsdefinition der Qualität im Gesundheitswesen muss die Qualität in der Medizin überprüft und bewertet werden.

Avedis Donabedian, ein „Begründer der modernen Qualitätssicherung in der Medizin“, unterteilt die Qualität in drei Dimensionen: Struktur-, Prozess- und Ergebnisqualität und trägt damit entscheidend zum Konzept der Qualitätsmessung und Beurteilung im Gesundheitswesen bei (Jaster 1997). Diese Unterteilung wird heute noch in der dargestellten Form zur Beurteilung der Versorgungsqualität herangezogen.

\section{Strukturqualität}

Die Strukturqualität beschreibt die Qualität der gegebenen statischen und personellen Voraussetzungen. Hierzu gehören die personellen, aber auch die technischen Voraussetzungen, die räumlichen Gegebenheiten und die Ablauforga- 
nisation. Zu den personellen Erfordernissen zählen nicht nur die Anzahl der Fachärzte, sondern auch deren Qualifikation. Es wird davon ausgegangen, dass in medizinischen Zentren, die eine gute "Organisationsstruktur", "qualifiziertes Personal" und eine gute "technische Ausstattung" vorweisen, sich "qualitativ hochwertige Erfolge erzielen lassen“" (Jaster 1997, S. 28).

\section{Prozessqualität}

Die Prozessqualität bezeichnet die Qualität des Behandlungsprozesses (Jaster 1997, S. 29). Hierzu zählen in erster Linie die organisatorischen Abläufe, die zwischen „Arzt”, „Patient“ und „Praxisteam" erfolgen: z. B. „Gesprächsführung“, "Anamneseerhebung“, „klinische Untersuchungen“, „Laboruntersuchungen“ und „Arzneimittelverordnung“ (Szecsenyi 1996, S. 47). Es wird davon ausgegangen, dass aus "qualitativ hochwertigen Behandlungsprozessen gute Behandlungsergebnisse" resultieren (Viethen 1995, S. 15).

\section{Ergebnisqualität}

Die Ergebnisqualität bezeichnet die Qualität des Behandlungsergebnisses (Viethen 1995). Die Bewertung des Behandlungsergebnisses wird vorgenommen anhand des "Gesundheits- und Zufriedenheitszustands des Patienten" (Viethen 1995, S. 15). Zu den Parametern, die Ergebnisqualität messbar machen können, werden u. a. „das Auftreten von postoperativen Komplikationen“, die „Wiederaufnahme des Patienten wegen der gleichen Erkrankung innerhalb von $30 \mathrm{Ta}-$ gen“ und die „Patientenzufriedenheit“" gezählt (Jaster 1997, S. 30).

Die Qualitätsdimensionen beeinflussen sich untereinander. Die vorhanden Strukturen und Ressourcen beeinflussen die Prozesse. Diese wiederum bestimmen die gemessene Ergebnisqualität mit (Schrappe 2001). Dass sich dieser Automatismus nicht immer ableiten lässt, zeigen Wissenschaftler in einigen Beispielen.

Viethen (1995) hinterfragt die Annahme, dass sich qualitativ hochwertige Behandlungsergebnisse aufgrund des Vorhandenseins technischer Möglichkeiten erzielen lassen. In sehr vielen Ländern, beispielsweise Osteuropas oder Ostasiens, erziele man gute Therapieergebnisse trotz einfachster technischer Möglichkeiten. Auch Szecsenyi macht darauf aufmerksam, dass die Betrachtung der Strukturqualität nur imstande sei, "grobe Mängel“ aufzudecken (Szecsenyi 1996, S. 47). Als Beispiel liefert er die Anwesenheit eines EKG-Gerätes in einer Praxis. Er hebt hervor, 
dass aus der Existenz eines EKG-Gerätes allein noch nicht auf die Behandlungsqualität eines Patienten mit koronarer Herzerkrankung (KHK) geschlossen werden könne. Viel wichtiger sei z. B. die Erkennung der Symptome einer Herzerkrankung oder die korrekte Interpretation des EKGs. Allerdings, so stellt Szecsenyi (1996) fest, können durch das Fehlen eines EKG-Gerätes in der Praxis grobe Versorgungsmängel entstehen.

Schrappe (2001) zeigt im folgenden einfachen Beispiel, dass eine schlechte Prozessqualität nicht unbedingt eine schlechte Ergebnisqualität zur Folge haben muss. Lange Wartezeiten in der Arztpraxis bedeuten zunächst eine schlechte Qualität in Hinblick auf die „Ablauforganisation“ und „Ausnutzung der zeitlichen bzw. finanziellen Ressourcen" (Prozessqualität) (Schrappe 2001, S. 268). Aus Ansicht älterer Patienten jedoch muss sich die schlechte Prozessqualität nicht auf die Patientenzufriedenheit (Ergebnisqualität) auswirken. Viele nutzen die Wartezimmer für Gespräche, die erst durch lange Wartezeiten zustande kommen. Diese „Befriedigung kommunikativer Bedürfnisse“, kann die Patientenzufriedenheit fördern (Schrappe 2001, S. 268). Darüber hinaus verbinden einige Patienten ein volles Wartezimmer mit einer guten Leistung des Arztes.

Den entscheidenden Schwachpunkt bildet laut Viethen jedoch die Nichtberücksichtigung der "hohen Variabilität des Patienten“ in der Aufteilung nach Donabedian (Viethen 1995, S. 15). Jaster stellt ebenfalls fest, dass die Ergebnisqualität sich wegen „der Komplexität von vielen Erkrankungen“ und den „individuellen Krankheitsabläufen" nur schwer zu vergleichenden Untersuchungen einsetzen lässt (Jaster 1997, S. 30). Darüber hinaus gibt Szecsenyi (1996) an, dass eine fehlende Compliance des Patienten ebenfalls Einfluss auf die Ergebnisqualität nimmt.

Nichtsdestotrotz bleibt die Donabedian-Trias ein bedeutendes Hilfsmittel bei der Beurteilung der Qualität im Gesundheitswesen.

\subsection{Schlafmedizin}

Mit dem Phänomen „Schlaf und Traum“ setzen sich die Menschen schon mehrere Jahrtausende auseinander. In der griechischen Mythologie verkörpert „Hypnos“ den Gott des Schlafes, der dem römischen Gott "Somnus" gleichzusetzen ist (nach Stuck et al. 2009, S. 2). Im 20. Jahrhundert rückt der Schlaf in die wissenschaftliche Auseinandersetzung. 1929 gelingt es dem Psychiater Hans Berger mit 
Hilfe des von ihm entwickelten Verfahren der Elektroenzephalographie (EEG), die hirnelektrischen Aktivitäten messbar zu machen und damit den Schlaf optisch darzustellen (Stuck et al. 2009). Die ersten Grundlagen für die Messung des Schlafes wurden gelegt.

Die Schlafmedizin als ein medizinisches Fachgebiet existiert erst seit einigen Jahren, jedoch mit einer großen klinischen Bedeutung. Neben dem Qualifikationsnachweis "Somnologie" der Deutschen Gesellschaft für Schlafforschung und Schlafmedizin (kurz DGSM) (DGSM 2009a) besteht seit 2004 die Zusatzbezeichnung "Schlafmedizin“ im Rahmen der neuen Weiterbildungsordnung der Ärztekammern als Ergänzung zu einer Facharztkompetenz (Stuck et al. 2009). Der Qualifikationsnachweis "Somnologie" bestätigt eine erfolgreiche Fortbildung in wissenschaftlicher und praktisch-klinischer Tätigkeit (DGSM 2009a) und befähigt den Arzt zur Durchführung schlafmedizinischer Diagnostik und Therapie.

Die Schlafmedizin setzt sich aus verschiedenen Fachgebieten zusammen und verlangt für ihre Diagnostik und Therapie „internistisch-pneumologische, kardiologische, neurologische, psychiatrische, psychologische und HNO-ärztliche Kenntnisse" (Stuck et al. 2009, S. 24).

Eine wichtige Voraussetzung zur diagnostischen und therapeutischen Behandlung verschiedener, den Schlaf betreffenden Erkrankungen lieferte die Einführung von Schlaflaboratorien. Diese existieren als ambulante und stationäre Einrichtungen. Dort werden Patienten auf vorliegende Schlafstörungen mit Hilfe der Anamnese, der allgemeinen körperlichen Untersuchung und spezieller Untersuchungstechniken, der Polysomnographie (PSG) (siehe Kapitel 1.3.1), untersucht.

\subsubsection{Polysomnographie im Schlaflabor}

Wie der Begriff andeutet, integriert die kardiorespiratorische Polysomnographie (PSG) mehrere Untersuchungen im Schlaf (siehe Abb. 1). Sie beinhaltet die Ableitungen von Hirnströmen mittels Elektroenzephalogramm (EEG), von Augenbewegungen mittels Elektrookulogramm (EOG), der Muskelaktivität an Bein und Kinn mittels Elektromyogramm (EMG) und die Ableitung des Herzschlages mittels EKG. Des Weiteren kommen atmungsbezogene Parameter hinzu: Schnarchmikrophon, Sauerstoffsättigung $\left(\mathrm{SaO}_{2}\right)$, Luftfluss an der Nase, thorakale und abdominelle 
Atembewegungen. Außerdem wird das Schlafverhalten des Patienten mit Hilfe einer Videokamera aufgezeichnet (Robert Koch-Institut 2005).

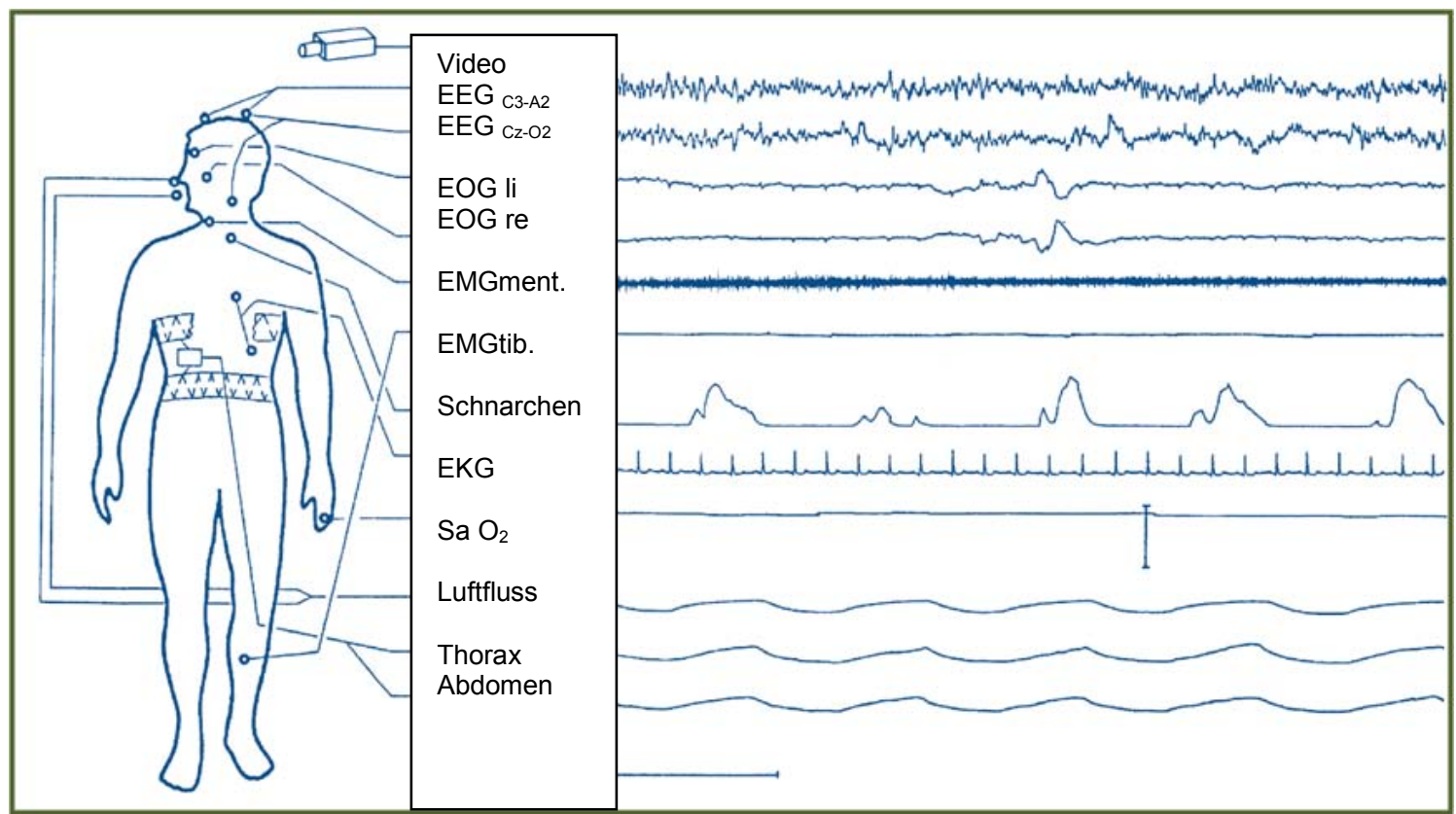

Abbildung 1. Kardiorespiratorische Polysomnographie (PSG) im Schlaflabor

Diese beinhaltet die Ableitungen von Elektroenzephalogramm (EEG), Elektrookulogramm (EOG), der Muskelaktivität an Bein und Kinn (Elektromyogramm, EMGtib, EMGment) und des Herzschlags (EKG). Darüber hinaus werden atmungsbezogene Parameter erfasst: Schnarchen, Sauerstoffsättigung $\left(\mathrm{SaO}_{2}\right)$, Luftfluss an der Nase, thorakale und abdominelle Bewegungen. Über Videokamera lässt sich das Schlafverhalten beobachten. (aus: Robert Koch-Institut (2005), S. 11, Abb. 2)

\subsubsection{Klassifikation der Schlafstörungen (ICSD-2)}

2005 publizierte die American Academy of Sleep Medicine (AASM) die 2. Version der International Classification of Sleep Disorders (ICSD-2). Diese klassifiziert Schlafstörungen in acht Hauptgruppen (vgl. Tab. 1).

Tabelle 1. Hauptgruppen der Schlafstörungen gemäß der American Academy of Sleep Medicine (AASM 2005)

\begin{tabular}{l}
\hline - Insomnien \\
- Schlafbezogene Atmungsstörungen \\
- Hypersomnische Störungen \\
- Zirkadiane Rhythmusstörungen \\
- Parasomnien \\
- Schlafbezogene Bewegungsstörungen \\
- Isolierte Symptome, Normvarianten, Ungelöstes \\
- Andere Schlafstörungen
\end{tabular}




\subsection{Qualitätssicherung und -förderung im Gesundheitswesen}

In der wissenschaftlichen und fachlichen Literatur werden die Begriffe Qualitätssicherung und Qualitätsmanagement oft als Synonym verwendet. Eine klare Differenzierung der Begriffe fehlt. Bereits 1995 wurde in der deutschen Übersetzung der DIN EN ISO-NORM 8402 gefordert, „die bisher als Oberbegriff verwendete Qualitätssicherung durch den Begriff Qualitätsmanagement zu ersetzen“. Der Begriff Qualitätssicherung konnte und musste deshalb neu definiert werden. Allerdings wurde diese Änderung im deutschen Gesundheitswesen nicht immer umgesetzt, sodass der Gebrauch des Begriffes "Qualitätssicherung" in unterschiedlichen Bereichen nicht kohärent ist (Selbmann 2001). In diesem Kapitel soll deshalb nur auf einzelne Definitionen verwiesen werden.

\subsubsection{Qualitätsmanagement}

Der Begriff „Qualitätsmanagement" stammt ursprünglich aus der Industrie und musste erst auf das Gesundheitswesen übertragen werden (Nüllen und Noppeney 2007). Das Ziel des Qualitätsmanagements ist die Qualitätsverbesserung im Sinne des Total Quality Management (TQM). TQM sei eine Form des Qualitätsmanagements, die sowohl alle Mitglieder als auch alle Bereiche einer Organisation (Abläufe, Prozesse, Ergebnisse) am Qualitätsgedanken beteilige (Nüllen und Noppeney 2007). Die kontinuierliche Verbesserung stellt den "Motor" vom Qualitätsmanagement dar (Köck 2001, S. 288).

Vor der Verbesserung steht der Schritt der Überprüfung. Durch die demographischen und epidemiologischen Veränderungen entwickle sich zunehmender Kostendruck, der das Gesundheitssystem zur Überprüfung der Effektivität und Effizienz der Leistungserbringung zwinge (Köck 2001). Mithilfe des Qualitätsmanagements sollen diese Überprüfungen verwirklicht werden.

Wichtiges und unverzichtbares Instrument des Qualitätsmanagements ist das Mittel der Qualitätssicherung (vgl. Kapitel 1.4.2). Qualitätssicherungsmaßnahmen sind notwendig zur Umsetzung des Qualitätsmanagements und umgekehrt (Schäfer 2001). 


\subsubsection{Qualitätssicherung}

Laut der Definition nach DIN EN ISO-Norm 9000:2000 ist Qualitätssicherung: „ein Teil des Qualitätsmanagements, der auf die Schaffung von Vertrauen darauf gerichtet ist, dass Qualitätsanforderungen erfüllt werden“. An dieser Definition lässt sich erkennen, dass Qualitätssicherung den Bereich des Qualitätsmanagements abdeckt, dessen Aufgabe darin besteht, die Umsetzung vorgegebener Qualitätsanforderungen zu prüfen. Noch einfacher ausgedrückt: Qualitätssichernde Maßnahmen werden mit dem Ziel durchgeführt, „zu kontrollieren, ob alles so gemacht wird, wie es gemacht werden soll“ (Nüllen und Noppeney 2007, S. 17).

Während, historisch gesehen, die Qualitätssicherung in der Industrie das Ziel der Überprüfung von Ergebnisqualität verfolgte, zielen heutige qualitätssichernde Maßnahmen auf die Überprüfung der Prozessqualität ab (Nüllen und Noppeney 2007).

Zur ältesten Qualitätssicherungsmaßnahme (1970) in Deutschland gehört die Perinatalerhebung zur Senkung der perinatalen Mortalität (Nüllen und Noppeney 2007).

\section{a) Gesetzliche Grundlage}

Der Gesetzgeber verpflichtet gemäß §135a SGB V Abs. 2 die Leistungsanbieter „Vertragsärzte, medizinische Versorgungszentren, zugelassene Krankenhäuser, Erbringer von Vorsorgeleistungen und Rehabilitationsmaßnahmen sich an einrichtungsübergreifenden Maßnahmen der Qualitätssicherung zu beteiligen, die insbesondere zum Ziel haben, die Ergebnisqualität zu verbessern" (SGB V 2011a). Der Gemeinsame Bundesausschuss (G-BA) wird gemäß §137 Abs. 1 mit der Aufgabe beauftragt, „die Qualität der durchgeführten diagnostischen und therapeutischen Leistungen" sowie "Mindestanforderungen an die Struktur-, Prozess- und Ergebnisqualität" zu definieren (SGB V 2011b). Die Umsetzung der Qualitätssicherung soll von einer "fachlich unabhängigen Institution“, die zuvor vom G-BA beauftragt wurde, erfolgen (SGB V 2011C).

Der Gesetzgeber verpflichtet zwar die Leistungserbringer im Gesundheitswesen zur Durchführung von Qualitätssicherung. Es gibt jedoch nur wenige Angaben da$\mathrm{zu}$, nach welchem Prinzip diese durchgeführt werden soll. Die wenigen Angaben beziehen sich vor allem auf die Erfassung der Strukturqualität (SGB V 2011b). 


\section{b) Einteilung der Qualitätssicherung}

Mittlerweile wird in der Fachliteratur zum Thema Qualitätssicherung zwischen traditionellen Verfahren der Qualitätssicherung sowie einer „externen“ und „internen“ Anwendung der Qualitätssicherung unterschieden (z. B. Selbmann 2001).

Traditionelle Verfahren der Qualitätssicherung werden oft nicht mehr als solche erkannt. Dazu zählen u.a. „Oberarzt- und Chefvisiten“, „Röntgenbesprechung“ oder "Komplikationsstatistik“ (Nüllen und Noppeney 2007).

Die Einordnung zwischen „extern“ und „intern“ ist zunächst einmal eine "Unterscheidung nach Ortsangaben", d. h. aus dem Winkel desjenigen, der die qualitätssichernden Maßnahmen in Auftrag gibt (Selbmann 2001, S. 274). Zur internen Qualitätssicherung gehören z. B. Maßnahmen, die von einer Station, einer Abteilung oder dem Krankenhaus zur Überprüfung der geleisteten Arbeit eingesetzt werden. Wird von außen eine Aufforderung zu qualitätssichernden Maßnahmen veranlasst (z. B. Gesetzgeber oder Krankenversicherungen), so spricht man von "externer" Qualitätssicherung (Selbmann 2001).

Ein wesentlicher Unterschied zwischen der internen und externen Qualitätssicherung besteht darin, dass externe qualitätssichernde Maßnahmen in Deutschland in der Regel verpflichtend sind und als Ziel die Kontrolle verfolgen. Interne Qualitätssicherungen sind in der Regel freiwillig und verfolgen das Ziel der Qualitätsförderung (Selbmann 2001).

\subsubsection{Richt- und Leitlinien als qualitätssichernde Maßnahme im Gesundheitswesen}

Zur Förderung der Qualität im Gesundheitssystem werden auch Richt- und Leitlinien eingesetzt. Leitlinien sind "systematisch entwickelte, wissenschaftlich begründete und praxisorientierte Entscheidungshilfen für die angemessene ärztliche Vorgehensweise bei speziellen gesundheitlichen Problemen“(AWMF 2007, S. 75). Leitlinien sollen „Entscheidungshilfen mit definierten Handlungsempfehlungen“ für beispielsweise Ärzte geben (Kopp et al. 2007, S. 89). Während die Anwendung von Richtlinien verpflichtend ist, kann von Leitlinien in bestimmten Situationen, aufgrund $z$. B. der Individualität des einzelnen Patienten, abgewichen werden ohne zivilrechtliche Verfolgung (AWMF 2007). 
Ziel sei einerseits die Verringerung der Varianz des diagnostischen und therapeutischen Vorgehens. Andererseits solle die medizinische Behandlung des individuellen Patienten verbessert werden (Bollschweiler 2001). Leitlinien sollen möglichst auf wissenschaftlichen Ergebnissen und dem aktuellen Stand der medizinischen Forschung basieren (AWMF 2007). Die Leitlinienerstellung kann auf diese Weise zur Erstellung von qualitätssichernden Maßnahmen beitragen (AWMF 2007). So können zur Bewertung der erreichten Qualität in gesundheitlichen Einrichtungen Leitlinienvorgaben benutzt werden.

In Deutschland ist in erster Linie die Arbeitsgemeinschaft der Wissenschaftlichen Medizinischen Fachgesellschaften (AWMF) für die Erstellung von Leitlinien zuständig (AWMF 2007).

\subsubsection{Verfahren zur Bewertung von Qualität im Gesundheitswesen}

Im Gesundheitswesen werden viele Verfahren zur Bewertung von Qualität angewendet. Die ausführliche Auseinandersetzung mit den vorhandenen Verfahren würde den Umfang dieser Arbeit übersteigen Deshalb sollen an dieser Stelle drei Verfahren kurz vorgestellt werden.

\section{a) Audits durch Peers}

Das Verfahren "Audits durch Peers" orientiert sich an der niederländischen „Visitatie“ der Fach- und Hausärzte (Kutschmann 2004, S. 16). Peers, meist Fachkollegen, beurteilen andere Peers anhand von vorgegebenen Bewertungsprotokollen vor Ort. Bewertet werden u.a. Behandlungsabläufe, Dokumentation und die Ergebnisse der Arbeit. Dabei wird weniger auf Strukturen und Ergebnisse als vielmehr auf die Kernprozesse der ärztlichen und pflegerischen Versorgung geachtet. Das Ergebnis sind nicht Zertifikate für die Öffentlichkeit, sondern ganz konkrete Vorschläge für die Verbesserung ärztlichen und pflegerischen Handelns (Selbmann 2001).

\section{b) Akkreditierung}

Grundsätzlich bestätigt der Begriff Akkreditierung eine „formale Überprüfung“ und Bewertung des „Ausmaßes an Erfüllung definierter Anforderungen“ (Schubert und Ebner 2001, S. 420). Im Gesundheitswesen bescheinigt die Bezeichnung „Akkreditierung" in der Regel eine externe Prüfung definierter Standards und Leitlinien 
und damit eine bestimmte Qualität in der Leistungserbringung (Schubert und Ebner 2001). Es bestätigt die „Kompetenz“, bestimmte Leistungen auf qualitativ hohem Niveau zu erbringen (Nüllen und Noppeney 2007, S. 101).

Die Erteilung einer Akkreditierung erfolgt entweder durch von Bundes- und Landesbehörden befugte Akkreditierungsstellen (Nüllen und Noppeney 2007) oder durch Fachgesellschaften, die nicht behördlich ermächtigt sind, interne Akkreditierungen zu erteilen. Die bekannteste Organisation im Gesundheitswesen, die unterschiedliche Akkreditierungsprogramme erarbeitet hat, ist die Joint Commission on Accreditation of Health Care Organizations (JCAHO) in den USA (Schubert und Ebner 2001).

\section{c) Zertifizierung}

Mit Zertifizierung wird ein „Prüfverfahren durch eine anerkannte Prüf- oder Zertifizierungsstelle" bezeichnet (Schubert und Ebner 2001, S. 420). Dabei wird die „Erfüllung von Anforderungen und Übereinstimmungen mit Normvorgaben wie beispielsweise der DIN EN ISO-Norm 9000 ff“" beurteilt (Schubert und Ebner 2001, S. 420). Eine Zertifizierung erfüllt vor allem den vertrauensbildenden Zweck (Nüllen und Noppeney 2007). Das Zertifizierungsverfahren besteht üblicherweise aus einer Vorprüfung, einer Bewertung der schriftlichen Unterlagen des Qualitätsmanagementsystems der Einrichtung und zusätzlich einem Zertifizierungsaudit, $d$. h. einer Prüfung durch das Zertifizierungsunternehmen (Schubert und Ebener 2001). Bei erfolgreicher Zertifizierung wird das Zertifikat beurkundet. Die Einrichtung darf von da an das Zertifizierungssiegel nach außen präsentieren (Nüllen und Noppeney 2007).

\subsubsection{Entwicklung von Qualitätsindikatoren zum Messen von Ver- sorgungsqualität}

Qualitätsindikatoren (Items) sind Instrumente der Qualitätssicherung und werden zur "Messung und Bewertung von Qualität" in der Gesundheitsversorgung eingesetzt (Blumenstock 2011, S. 154). Diese stellen indirekt als Parameter mit Hilfe von „Zahlenwerten bzw. Zahlenverhältnissen“ die Versorgungsqualität dar (Jäckel 2009, S. 2). Zuvor muss ein Referenzbereich definiert werden, innerhalb dessen sich die Bewertung durch den Qualitätsindikator bewegt (Jäckel 2009). Die Definition des Referenzbereiches und der Qualitätsindikatoren sollte sich an evidenz- 
basierten Untersuchungen, beispielsweise Leitlinien, orientieren oder, wenn dies nicht möglich ist, auf Konsensbildung erfahrener Experten zurückgreifen (Mainz 2003).

Wie auch im Kapitel 1.1. dargestellt, gibt es zahlreiche Definitionen des Qualitätsbegriffes in der Medizin. Diese Tatsache erschwert die Entwicklung von geeigneten Instrumenten zur Messung der nicht direkt fassbaren Qualität.

Um die Qualität der Qualitätsindikatoren zu überprüfen, müssen zunächst Anforderungen an diese formuliert werden. Wichtige internationale Definitionen für Qualitätsanforderungen an Qualitätsindikatoren werden von Geraedts et al. (2003) aufgearbeitet. Nur ein Beispiel soll im Folgenden gegeben werden, das einige zentrale Anforderungen an Qualitätsindikatoren formuliert. Sens et al. verfassten 2007 dazu die sogenannte RUMBA-Regel. Der zufolge müssen Indikatoren für einen Problembereich wichtig (Relevant), verständlich (Understandable), messbar mit hoher Reliabilität und Validität (Measurable), durch Verhalten beeinflussbar (Behaviourable) und realistisch erreichbar und praktikabel (Achievable and feasible) sein (Sens et al. 2007, S. 30).

\subsubsection{Entwicklung der Qualitätsindikatoren für den Fachbereich Schlaf- medizin}

Die Formulierung von Qualitätsindikatoren im Rahmen der Überprüfung der Prozessqualität in der Schlafmedizin muss viele Besonderheiten berücksichtigen, die die Definition der Qualitätsindikatoren erschweren. Während in Fachgebieten wie z. B. der Kardiologie direkte Qualitätsindikatoren zur Messung der Versorgungsqualität bestimmt werden können, ist dies wegen der interdisziplinären Ausrichtung der Schlafmedizin nur bedingt möglich. Kann z. B. zur Darstellung der Prozessqualität in der Kardiologie die Rate der Patienten mit Myokardinfarkt und anschließend erhaltener Thrombolysetherapie erfasst werden (Mainz 2003), ist es in der Schlafmedizin angesichts der hohen Anzahl unterschiedlicher Erkrankungen des Schlafes aus diversen Fachdisziplinen nicht möglich. Zusätzlich ergibt sich aus der gesundheitspolitischen Lage, in der die Polysomnographie als Kontrolluntersuchung im Schlaflabor nicht vergütet wird, eine andere Schwierigkeit. Der Ablauf des Prozesses beziehungsweise der Erfolg der Behandlung können nur bedingt beurteilt werden. 
Auch die Entwicklung von Indikatoren, die die Ergebnisqualität erfassen, ist in der schlafmedizinischen Qualitätssicherung wegen den bereits oben erörterten Gründen nur bedingt möglich. Indikatoren wie z. B. die Zunahme der Schlafeffizienz können nicht direkt beurteilt werden, wie dies bei anderen Qualitätssicherungsprogrammen möglich ist (z.B. „Postoperative Beweglichkeit“ als Qualitätsindikator nach einer Knie-Totalendoprothese (BQS-Institut 2008)).

\subsection{Qualitätsbezogene Aktivitäten der DGSM in der deutschen Schlafmedizin}

Die Deutsche Gesellschaft für Schlafforschung und Schlafmedizin (DGSM) beschäftigt sich als wissenschaftliche Fachgesellschaft mit der Schlafforschung und der Schlafmedizin (DGSM 2007). Neben den wissenschaftlichen und praktischen Einsätzen im Fachbereich Schlafmedizin etablierte die DGSM ein Qualitätssicherungsverfahren zur Überprüfung und Verbesserung der schlafmedizinischen Behandlung des Patienten (DGSM 2007). Dies geht auf eine langjährige Tradition zurück und führte zur Entwicklung unterschiedlicher Verfahren.

\subsubsection{Bisherige Verfahren zur Überprüfung der Versorgungs- qualität}

\section{a) Akkreditierungsverfahren: Strukturqualität}

Seit 1992 überprüft die DGSM im Rahmen eines Akkreditierungsverfahrens die Strukturqualität. Im „Leitfaden für Akkreditierung“ von Penzel et al. (2000) wird das Prinzip der Akkreditierung vorgestellt. Es beruht auf einer Ortsbegehung mit Begutachtung durch eine interdisziplinär zusammengesetzte Gruppe von drei Experten und einem zuvor auszufüllenden Fragebogen. Dieser besteht aus Fragen zum angestellten Personal, den Geräten und Räumlichkeiten sowie der schlafmedizinischen Leistungen (DGSM 2009b). Im Dialog mit dem Leiter des Schlaflabors und dem technischen Personal findet mit Hilfe des ausgefüllten Fragebogens die Bewertung statt. Schließlich erstellen die Gutachter ein Protokoll mit Empfehlungen zur Verbesserung der Qualität und entscheiden über die Ablehnung oder Empfehlung zur Akkreditierung des Labors.

Nach erfolgter Akkreditierung wurde eine schriftliche Reevaluation der Laboratorien im Abstand von zwei Jahren auf Grundlage eines Fragebogens vorgenommen.

Bis heute wird dieses Verfahren zur Akkreditierung eines neuen Schlaflaboratoriums verwendet. Jedoch wird die Reevaluation durch ein Qualitätssicherungspro- 
gramm ersetzt (DGSM 2011). Das vorgestellte Akkreditierungsverfahren der DGSM orientiert sich an der Überprüfung und Beurteilung der Strukturqualität.

\section{b) Qualitätssicherungsverfahren: Prozessqualität}

Seit 2000 kommt eine weitere Dimension des Qualitätsbegriffs nach Donabedian zusätzlich zur Überprüfung: die Prozessqualität. Dazu entwickelt die DGSM im Jahre 2000 ein Qualitätssicherungsprogramm (Fischer et al. 1999). Dieses wird zur Untersuchung der Prozessqualität in DGSM-akkreditierten Schlafzentren im Jahre 2000 und in leicht umgewandelter Form im Jahre 2002 eingesetzt (Kutschmann et al. 2002).

Das zugrundeliegende Verfahren zur Überprüfung der Prozessqualität in den bei der DGSM akkreditierten Schlaflaboratorien ist das sogenannte „Peer-ReviewVerfahren“. Dieses beruht darauf, dass unabhängige Gutachter („Peers“) den Ablauf im Schlafzentrum anhand von Patientenakten begutachten (,reviewed“). Als Grundlage zur Beurteilung der Akten liegt eine vorgegebene „Checkliste qualitätsrelevanter Prozessmerkmale" vor. Hinzu kommt ein Manual mit entsprechenden Beurteilungskriterien (Kutschmann et al. 2002).

\section{c) Ergebnisqualität}

Zusätzlich wurde neben der Überprüfung der Struktur- und Prozessqualität im Jahr 2002 die Messung der Ergebnisqualität im Rahmen eines Pilotprojekts durchgeführt. Hierbei wurden Daten von Patienten in Form eines Fragebogens zu subjektiver Symptomatik, psychischer Befindlichkeit, sozialen Problemen, Therapiezielvorstellungen, subjektiver Erreichung des Therapieziels, Zufriedenheit mit Leistungserbringern, Bereitschaft zur Selbstbeteiligung und zu Problemen mit Kostenträgern erhoben (DGSM 2009c).

\subsubsection{Aktuelle qualitätsbezogene Aktivitäten der DGSM: Verfah- rensänderung}

Das aktuelle Qualitätssicherungsprogramm 2009/2010 vereint zwei der bisher durchgeführten Verfahren: das Akkreditierungsverfahren und das Peer-ReviewVerfahren zur Messung der Prozessqualität. Bisher erfolgte im Abstand von zwei Jahren nach erfolgreicher Akkreditierung des Schlaflaboratoriums eine schriftliche Reevaluation zur Weiterführung der Akkreditierung (Penzel et al. 2000). Ab 2009 ersetzt die erfolgreiche Teilnahme am Qualitätssicherungsverfahren der DGSM 
die schriftliche Reevaluation und verlängert damit die Akkreditierung um weitere zwei Jahre (DGSM 2011). Während die Teilnahme am Qualitätssicherungsverfahren im Jahre 2000 und 2002 auf freiwilliger Basis erfolgte (Kutschmann et al. 2002), ist das aktuelle Qualitätssicherungsverfahren inzwischen für jedes akkreditierte schlafmedizinische Zentrum verpflichtend. Vorausgesetzt es strebt eine weitere DGSM-Akkreditierung an (DGSM 2011).

Dementsprechend war es nötig, das bisherige Verfahren zur Messung der Prozessqualität aus den Jahren 2000 bzw. 2002 zu überarbeiten und den neueren Gegebenheiten der schlafmedizinischen Praxis anzupassen. Auf diese Weise konnte ab 2009 die gegenwärtige Qualität in den DGSM-akkreditierten Schlaflaboratorien erfasst werden. Die Analyse dieser Ergebnisse soll zunächst einen Überblick darüber verschaffen, wie sich die aktuelle Qualität der Schlaflaboratorien darstellt. Diese Analyse ist ein Teil der folgenden Untersuchungen.

Es wurde versucht, für die vorliegenden Durchgänge der Qualitätssicherung das Verfahren in der Organisation zu vereinfachen. Anders als im ursprünglichen Verfahren, in dem vollständige Patientenakten die Beurteilungsbasis bildeten (Kutschmann et al. 2002), erfolgte die Begutachtung nur noch anhand eines Deckblatts mit allgemeinen Informationen zum Labor, zu den Mitarbeitern und den Geräten (Strukturqualität) und eines Entlassungsberichtes einschließlich der zugehörigen Nachtprotokolle (Prozessqualität). Auf diese Weise vereint das überarbeitete Qualitätssicherungsprogramm sowohl die Prüfung der Struktur- als auch der Prozessqualität. Zum anderen variiert die Anzahl der geprüften Patientenakten. Während aktuell die Beurteilung anhand von drei Patientenakten erfolgte, waren es im ursprünglichen Verfahren sechs bzw. sieben (Kutschmann et al. 2002).

Darüber hinaus sollten im Qualitätssicherungsprogramm 2009/10 zwei verschiedene Verfahren bezüglich der Auswahl der Patientenakten erprobt werden. In dieser Arbeit werden die Erhebungsverfahren folgendermaßen genannt: das "Stichtagsverfahren“ und das „Randomisierte Verfahren“. Im Stichtagsverfahren werden nach Bekanntgabe eines definierten Datums die Schlaflaboratorien aufgefordert, retrospektiv die Patientenakten einzuschicken. Im Randomisierten Verfahren werden die im Zeitraum von 14 Tagen aufgenommenen Patienten zunächst auf einer gesonderten Liste (prospektiv) aufgeführt. Aus dieser Patientenliste wer- 
den durch die DGSM-Verantwortlichen randomisiert drei Patientenakten ausgewählt. Eine Evaluation beider Verfahren wird notwendig.

Die "Checkliste qualitätsrelevanter Prozessmerkmale“ wurde 1997 erstmals von einem Gremium anerkannter Experten im Bereich der Schlafmedizin erarbeitet (Kutschmann et al. 2002, Fischer et al. 1999). Im aktuellen Qualitätssicherungsprogramm wurde diese entsprechend gekürzt und überarbeitet. Den einzelnen Versionen der Checkliste liegt jedoch ein gemeinsamer Aufbau zugrunde (Fischer et al. 1999). Es wird in fünf Beurteilungsfelder (Anamnese, Diagnostik, Diagnosesicherung, Therapie und Entlassungsbericht) unterteilt, die jeweils aus qualitätsrelevanten Prozessmerkmalen, den sogenannten „Items“, bestehen.

Die Eignung der damaligen Qualitätsindikatoren (Items) als Instrument zur Beurteilung der Prozessqualität wurde von Kutschmann (2004) anhand der Indikatorreliabilität und Indikatorvalidität überprüft (Kutschmann 2004).

Insgesamt erschienen die meisten Items reliabel, vor allem jene, die sich auf allgemeine Aspekte der Prozessabläufe im Schlaflabor beziehen. Zu nennen sind „Allgemeine Anamnese“, „Allgemeine klinische Untersuchung“, und „Allgemeine Behandlungsmaßnahmen" (Kutschmann 2004, S. 129). Obwohl einige Items aus der alten Checkliste übernommen werden konnten, mussten auch neue definiert werden.

Es ist wichtig, Qualitätssicherungsverfahren nicht nur zu entwickeln, sondern auch zu überprüfen und zu optimieren. Kutschmann (2002) zeigt in Abbildung 2 den Zusammenhang zwischen Peer-Review-Verfahren und dem „Plan-Do-Check-ActZyklus" (PDCA). In der "Check-Phase“ des Zyklus (Peer-Review-Verfahren) werden die Daten erhoben. Deren Beurteilung dient zum einen der „Verbesserung der Prozessqualität“ in den akkreditierten Schlaflaboratorien („Act-Phase“). Zum anderen ist sie Ausgangspunkt für die "Optimierung der Beurteilungsinstrumente und Verfahrensdurchführung" in einer "Plan“-Phase (Kutschmann et al. 2002, S. 89). Diese Optimierung der Instrumente wird an die Schlaflabore zurückgemeldet, damit die Schlaflaboratorien diese in der „Do“-Phase umsetzen können (Kutschmann et al. 2002). Die Erkenntnisse der vorliegenden Arbeit sollen genau in der „Plan“Phase hilfreich werden. Die Beurteilungsinstrumente im DGSM-Qualitätssicherungsverfahren sollen optimiert werden. 


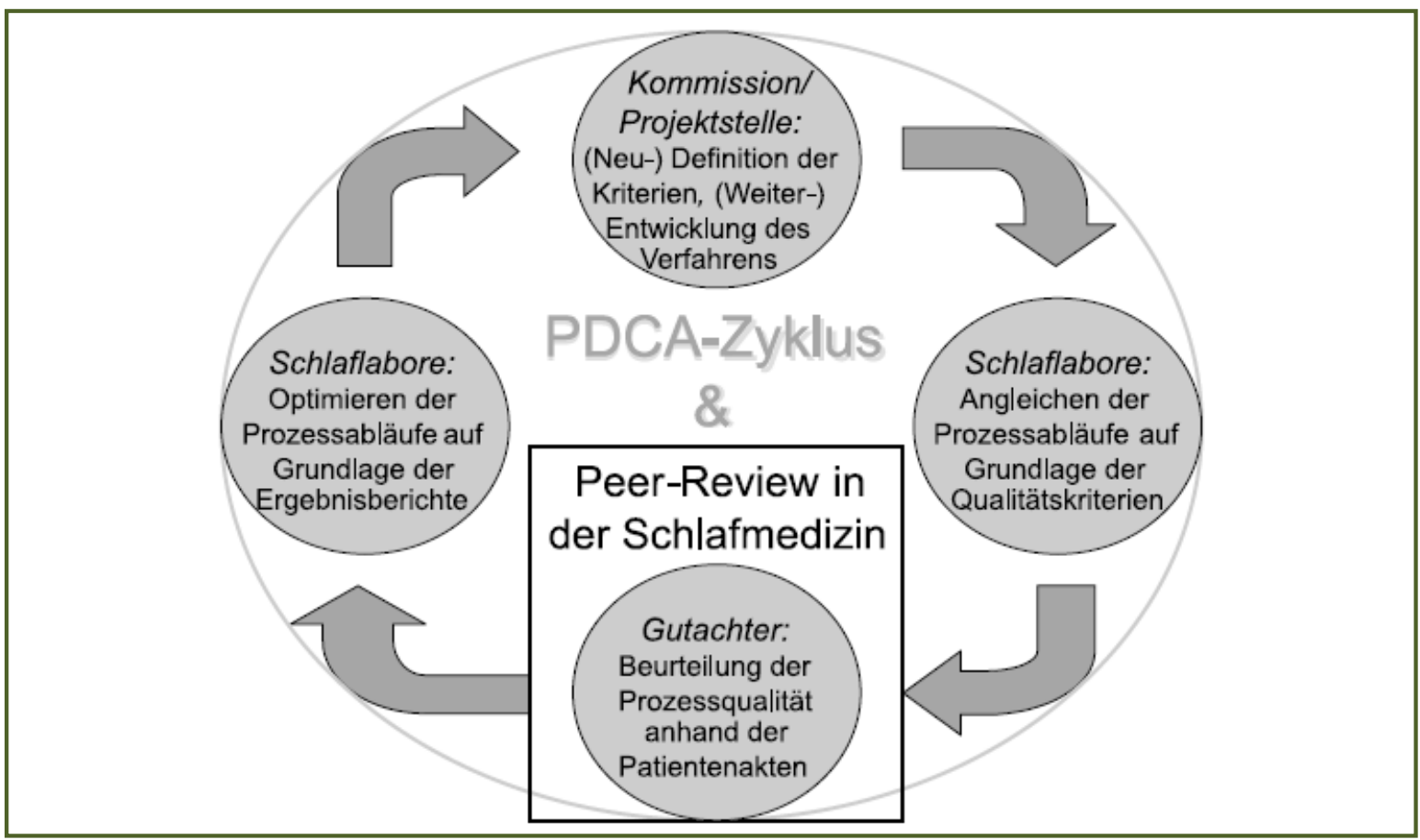

Abbildung 2. Zusammenhang des „Plan-Do-Check-Act (PDCA) Zyklus“ und des Peer-Review-Verfahrens der DGSM (aus Kutschmann et al. 2002, S. 91)

Auf der Suche nach geeigneten Qualitätskriterien (Items) zur Messung und Abbildung der Prozessqualität wäre es interessant, nicht nur die Items selbst zu überprüfen, sondern den Umgang der Gutachter mit innen. Es ist aufschlussreich zu wissen, welche Items den Gutachter zu einer Revisitation veranlassen. Nur weil gewisse Items durch die Vorgabe von Expertenmeinung zur Verfügung gestellt wurden, ist für das DGSM-Qualitätssicherungsprogramm noch nicht untersucht worden, ob die Gutachter wirklich nach diesen vorgegebenen Qualitätskriterien entscheiden. Es besteht weiterhin die Frage nach dem Einfluss der Subjektivität auf das Urteil des Gutachters. Aus dieser Fragestellung ergibt sich die Aufgabenstellung nach der Suche und Neuentwicklung möglichst objektiver Items. An dieser Stelle kann die vorliegende Dissertation nur den Hinweis für eine solche Untersuchung liefern. Eine Beantwortung muss in einer nachfolgenden separaten Bearbeitung erfolgen. 


\section{Fragestellung}

Im deutschen Gesundheitswesen wurden in den letzten Jahren sowohl Qualitätssicherungsprogramme als auch Qualitätsmanagementaktivitäten zunehmend eingeführt (Glattacker und Jäckel 2007).

Aus den bisherigen Ausführungen wird deutlich, dass einerseits die Gesundheitspolitik Qualitätssicherung auf verschiedenen Ebenen vorschreibt, andererseits aber oft entsprechende Organisationsstrukturen und vor allem inhaltliche Vorgaben, insbesondere zu Prozess- und Ergebnisqualität, fehlen und erst noch erarbeitet werden müssen. Die DGSM hat bereits vor circa 10 Jahren begonnen, die Prozessqualität akkreditierter Schlaflabore zu erfassen und die Teilnahme mittlerweile als verpflichtenden Bestandteil der Weiterakkreditierung etabliert.

Die vorliegende Arbeit soll auf Grundlage der zwei bislang abgeschlossenen Qualitätssicherungsdurchgänge folgende Fragestellungen bearbeiten:

1) Zu welchen Ergebnissen kommen die angewandten methodischen Verfahren Stichtagsverfahren und Randomisiertes Verfahren - hinsichtlich des Organisationsablaufs und welche Vor- und Nachteile haben sie im Vergleich? Einschließlich der Prüfung, ob das methodische Verfahren einen Einfluss auf den Ausgang des Ergebnisses bezüglich der Akkreditierung bzw. auf das Verhalten des Gutachters bezüglich Revisitationsempfehlung hat? Hierzu sollen die Einzelitems getrennt für anschließend weiter akkreditierte und zu revisitierende Labore ausgewertet werden.

2) Wie ist die Qualität der durch die DGSM-akkreditierten teilnehmenden Schlafzentren zu bewerten? Welche Mängel in den Beurteilungsfeldern sind offensichtlich und wie ist die Auswirkung auf die Weiterakkreditierung? Dazu soll die kategorisierte Bewertung jedes Items durch die Gutachter computergestützt erfasst und vergleichend dargestellt werden.

3) Lassen sich Items bestimmen, von denen die Gutachterempfehlungen zur Revisitation in hohem Maße abhängen? Welche Items eignen sich als Qualitätsindikatoren von Prozessmerkmalen in interdisziplinären Fachgebieten? 


\section{Methodik}

Im Verfahren 2009/10 zur Überprüfung der Prozessqualität in den DGSMakkreditierten Schlaflaboratorien wurde sowohl die „Checkliste qualitätsrelevanter Prozessmerkmale" modifiziert als auch der organisatorische Ablauf verändert. Es wurden zwei unterschiedliche Verfahren bezüglich der Auswahl der Patientenakten angewandt (siehe unten). Das aktuelle Konzept der Qualitätssicherung ersetzt die regelhafte Reevaluation der akkreditierten Schlafzentren im Abstand von zwei Jahren und entscheidet über das Fortbestehen der Akkreditierung des Schlaflabors (DGSM 2011).

\subsection{Teilnehmende Schlaflaboratorien}

Schlaflaboratorien, die zum Zeitpunkt der durchgeführten Qualitätssicherung zur Reevaluation standen, wurden schriftlich zur Teilnahme eingeladen. Im Stichtagsverfahren wurden 169 der bei der DGSM akkreditierten schlafmedizinischen Zentren angeschrieben. Im Randomisierten Verfahren wurden 176 Schlaflaboratorien angeschrieben. 149 der von der DGSM akkreditierten Schlaflaboratorien nahmen am Stichtagsverfahren und 128 am Randomisierten Verfahren teil. In die Analyse der Arbeit gingen 118 Schlaflaboratorien des Randomisierten Verfahrens ein. Tabelle 2 zeigt die einzelnen Fachbereiche der teilnehmenden Schlaflaboratorien.

\subsection{Verfahren}

Die Überprüfung der Struktur- bzw. Prozessqualität in den akkreditierten Schlafzentren der DGSM erfolgt im Rahmen eines sogenannten „Peer-ReviewVerfahrens“. Gutachter („Peers“) beurteilten die Qualität der akkreditierten Schlaflaboratorien. Dies erfolgte im DGSM-Qualitätssicherungsverfahren anhand von Patientenakten (vgl. Kap. 2.4).

Tabelle 2. Am Qualitätssicherungsverfahren teilnehmende Schlaflaboratorien mit ihrem Schwerpunkt im Fachbereich

\begin{tabular}{|c|c|c|}
\hline Fachabteilung & $\begin{array}{c}\text { Anzahl der Schlaflaboratorien- } \\
\text { Stichtagsverfahren }\end{array}$ & $\begin{array}{c}\text { Anzahl der Schlaflaboratorien- } \\
\text { Randomisiertes Verfahren }\end{array}$ \\
\hline Innere Medizin & 104 & 93 \\
\hline Neurologie/Psychiatrie & 31 & 9 \\
\hline Pädiatrie & 12 & 10 \\
\hline Hals-Nasen-Ohren Heilkunde & 2 & 6 \\
\hline
\end{tabular}




\subsection{Organisatorischer Ablauf}

Es wurden zwei unterschiedliche organisatorische Erhebungsverfahren in Bezug auf die Auswahl der zu überprüfenden Patientenakten erprobt.

\subsubsection{Stichtagsverfahren}

Zur Datenerhebung wurden zunächst im Frühjahr 2009 (24.01.2009) die 169 DGSM-akkreditierten Schlaflaboratorien, deren Akkreditierung Ende des Jahres 2008 auslief, angeschrieben. Dieses Schreiben umfasste ein sogenanntes Deckblatt zur Erfassung der allgemeinen Angaben zu Labor, Mitarbeitern und den im letzten Kalenderjahr durchgeführten Untersuchungen. Des Weiteren lag eine Checkliste mit deren Ausführungsbestimmungen und ein Musterprotokoll für die Nächte bei. Es wurde darum gebeten, die Dokumentation der Akten an die Erfordernisse der Checkliste für den vierwöchigen Zeitraum vom 01.03-31.03.2009 anzupassen. Darüber hinaus wurde mitgeteilt, dass ein retrospektiver Stichtag aus dem genannten Zeitraum angesetzt werden wird.

Im April wurde schließlich ein retrospektiver Stichtag festgelegt und den Schlaflaboratorien mitgeteilt. Diese waren dann aufgefordert Akten der ersten drei Patienten, die ab diesem festgelegten Zeitpunkt in dem Schlaflabor therapiert wurden, zu pseudonymisieren und an die DGSM zu schicken. Im Anschluss wurden die Patientenakten zur Begutachtung an die Gutachter verteilt.

\subsubsection{Randomisiertes Verfahren}

Die 176 Schlafzentren, deren Akkreditierung zum Ende des Jahres 2009 auslief, wurden im Herbst 2009 (15.10.2009) angeschrieben. Dem Schreiben lag wie im Frühjahr 2009 ein Deckblatt, eine Checkliste mit deren Ausführungsbestimmungen und ein Musterprotokoll bei.

Bei diesem Durchgang der Datenerhebung waren die Labore aufgefordert, ab dem 30.10.2009 die nächsten folgenden 20 im Schlaflabor behandelten Patienten bzw. über einen Zeitraum von 14 Tagen aufgenommenen Patienten auf einer gesonderten Liste aufzuführen. Dabei war darauf zu achten, dass ambulant aufgenommene Patienten oder solche, die nur zur Kontrolle kommen, nicht in die Liste aufgenommen werden. Die Patientenliste enthielt Angaben zum Behandlungsdatum, Geburtsdatum, zu den Initialen und den durchgeführten diagnostischen und therapeutischen Maßnahmen. 
Aus dieser Liste wurden drei Patienten von einem Verantwortlichen der DGSM randomisiert ausgesucht. Die dazugehörigen Patientenakten wurden angefordert. Schließlich erfolgte die Verteilung an die drei dem Labor zugeteilten Gutachter.

\subsection{Patientenakten}

Die Begutachtung erfolgte anhand von Patientenakten, bestehend nur aus dem Entlassungsbericht, einem Deckblatt und aus einem Nachtprotokoll. Das Deckblatt erfasste die allgemeinen Angaben zum Schlaflabor, die Ausstattung der Geräte und die personelle Besetzung sowie deren Qualifikation.

Zur Begutachtung lag jedem Gutachter eine Patientenakte pro Schlaflabor vor. Insgesamt wurden drei Akten pro Schlaflabor bewertet.

Aus Datenschutzgründen mussten alle Patientenunterlagen pseudonymisiert sein.

Das Nachtprotokoll sollte u. a. neben der Aufführung der Namen sowohl der Sitzwache als auch des Arztes eine Übersicht der ablaufenden Nacht mit einer genauen Zeitangabe liefern. Die ausführliche Beschreibung des Nachtprotokolls kann im Anhang 8.4 und 8.5 nachvollzogen werden.

Im Entlassungsbericht sollte neben einer ausführlichen Anamnese, bereits durchgeführter Diagnostik und Medikationsauflistung auch die Therapie bzw. die Therapieempfehlung diskutiert werden.

\subsection{Gutachter}

Es nahmen insgesamt 34 Gutachter an dem Projekt teil. Diese stammen aus unterschiedlichen medizinischen Fachbereichen: HNO (1), Pädiatrie (3), Pneumologie (15), Psychiatrie (9) und Neurologie (6). Das Besondere dabei war, dass es sich bei der Auswahl der Gutachter nicht um Externe handelte, sondern um Leiter oder deren Vertreter der akkreditierten Laboratorien.

Zur Beurteilung eines Schlafzentrums wurden drei unterschiedliche Gutachter zufallsmäßig ausgewählt, um der subjektiven Bewertung durch einen einzelnen Gutachter vorzubeugen. Dabei wurde darauf geachtet, dass mindestens einer der Gutachter aus dem gleichen medizinischen Fachgebiet wie das zu beurteilende Schlaflabor stammt, um fachgebietsspezifische Problematiken zu erkennen und zu bewerten. In pädiatrischen Schlaflaboratorien bewerteten jeweils drei Pädiater. 


\subsection{Die Checkliste qualitätsrelevanter Prozessmerkmale und das Manual mit den Beurteilungskriterien}

Die im Jahr 1998 erarbeitete "Checkliste qualitätsrelevanter Prozessmerkmale“ (Fischer et al. 1999) wurde für das Qualitätssicherungsverfahren 2009/10 durch anerkannte Experten mit langjähriger Erfahrung in der schlafmedizinischen Versorgung modifiziert und optimiert. Die Aufteilung der Checkliste in Beurteilungsfeldern und einige Items konnten in der Neufassung übernommen werden. Aufgrund wissenschaftlicher Fortschritte und der gesundheitspolitischen Entwicklung in den letzten Jahren war es darüber hinaus notwendig, zur Überprüfung der Prozessqualität neue Items zu entwickeln.

Die Version der Checkliste qualitätsrelevanter Prozessmerkmale im Jahr 2009/10, die zur Überprüfung der Prozessabläufe eingesetzt wurde, bestand aus fünf Beurteilungsfeldern: Anamnese, Diagnostik, Diagnosesicherung, Therapie und Entlassungsbericht._Diese Beurteilungsfelder wiederum wurden nochmals in einzelne Prozessmerkmale (Items) unterteilt, die jeweils vom Gutachter auf Grundlage des Entlassungsberichts und des Nachtprotokolls geprüft werden mussten. Die Checkliste im DGSM-Peer-Review-Verfahren 2009/10 bestand aus insgesamt 37 Items. Zur Beurteilung der Prozessmerkmale wurden dem Gutachter definierte Antwortkategorien vorgegeben (siehe Tab. 3).

Tabelle 3. Definierte Antwortmöglichkeiten zur Beurteilung der Prozessmerkmale

\begin{tabular}{|c|c|c|c|c|c|c|c|c|}
\hline Beurteilungsfeld & \multicolumn{8}{|c|}{ Antwortkategorie } \\
\hline Anamnese & \multicolumn{2}{|c|}{ vorhanden } & \multicolumn{4}{|c|}{ nicht vorhanden } & \multicolumn{2}{|c|}{ bereits extern durchgeführt } \\
\hline Diagnostik & vorhanden & $\begin{array}{c}\text { nicht } \\
\text { vorhanden }\end{array}$ & $\begin{array}{l}\text { ber } \\
\text { dur }\end{array}$ & $\begin{array}{l}\text { sextern } \\
\text { geführt }\end{array}$ & & $\begin{array}{l}\text { Sehlung } \\
\text { erfolgt }\end{array}$ & $\begin{array}{l}\text { Empfehlung } \\
\text { fehlt }\end{array}$ & $\begin{array}{l}\text { entfällt/nicht } \\
\text { erforderlich }\end{array}$ \\
\hline Diagnosesicherung & \multicolumn{8}{|c|}{ uneinheitliche Struktur (siehe Abb. 3.) } \\
\hline Therapie & durchgeführt & \multicolumn{2}{|c|}{ terminiert } & \multicolumn{2}{|c|}{ empfohlen } & fehlt & \multicolumn{2}{|c|}{ entfällt/nicht erforderlich } \\
\hline Entlassungsbericht & angemessen & $\begin{array}{r}\text { teilwe } \\
\text { angeme }\end{array}$ & & $\begin{array}{r}\text { nich } \\
\text { angeme }\end{array}$ & & fehlt & entfällt/nic & erforderlich \\
\hline
\end{tabular}


Für das Beurteilungsfeld Anamnese standen drei Kategorien zur Verfügung. Für das Beurteilungsfeld Diagnostik waren es sechs. Die Beurteilungsfelder Therapie und Entlassungsbericht wurden in fünf Kategorien unterteilt. Die vorgegebenen Antwortkategorien des Beurteilungsfeldes Diagnosesicherung wiesen einen uneinheitlichen Aufbau auf (vgl. Abb. 3).

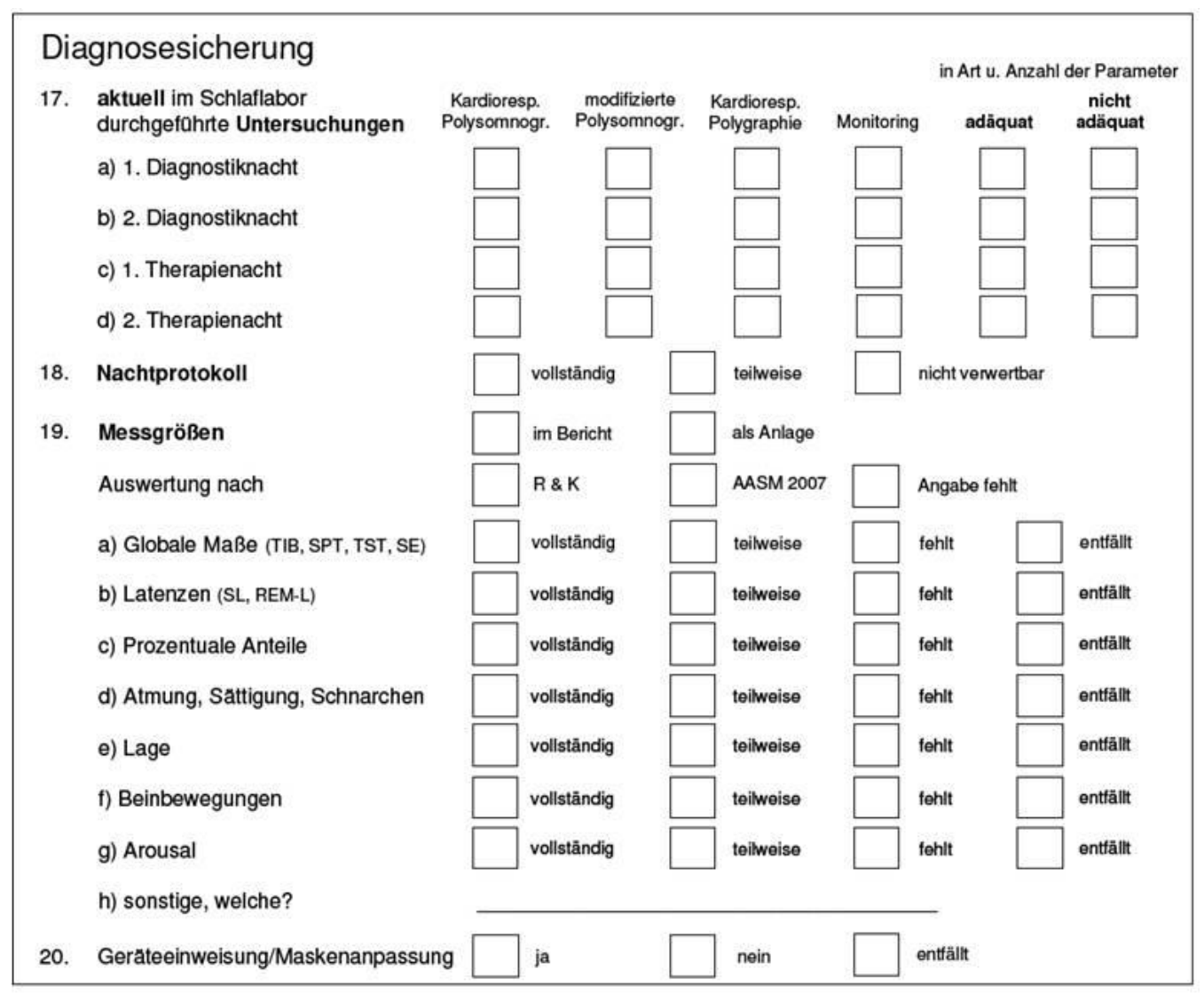

Abbildung 3. Beurteilungsfeld Diagnosesicherung der Checkliste qualitätsrelevanter Prozessmerkmale im DGSM-Peer-Review-Verfahren 2009/2010

Zur Veranschaulichung des Aufbaus der Beurteilungsfelder ist das Beurteilungsfeld Anamnese beispielhaft in Abbildung 4 gezeigt. Die vollständige Checkliste und das Manual können im Anhang 8.1 nachvollzogen werden 


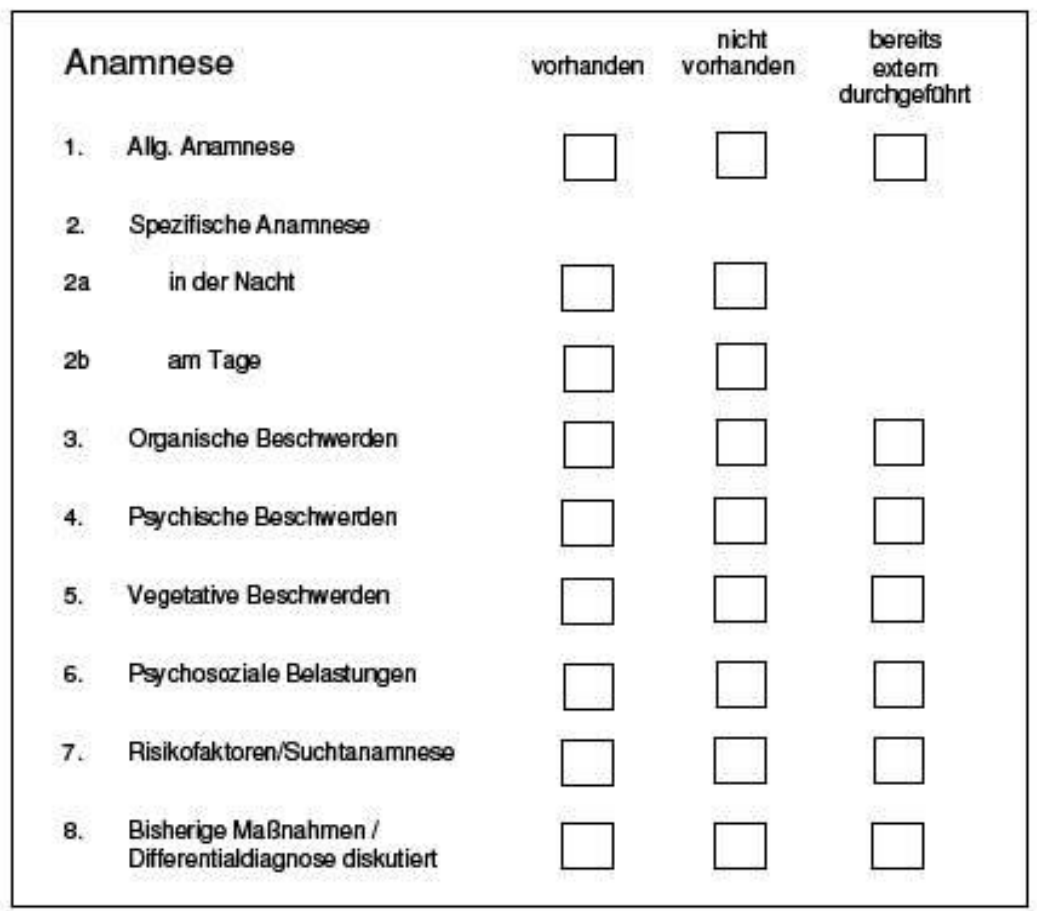

Abbildung 4. Beurteilungsfeld Anamnese der Checkliste qualitätsrelevanter Prozessmerkmale im DGSM-Peer-Review-Verfahren 2009/2010

Die Checkliste wurde abgeschlossen mit einer visuellen Analogskala (VAS). Mit dieser Analogskala sollte der Gutachter beurteilen, ob der Gesamtprozess dem aktuellen Stand wissenschaftlicher Erkenntnisse, Empfehlungen und Leitlinien entspricht. Die dimensionslose Skala beginnt mit dem Zahlenwert „0“ für „überhaupt nicht“ und reicht in 20er Schritten bis „100“ für „Vollständig“ (siehe Abb. 5).

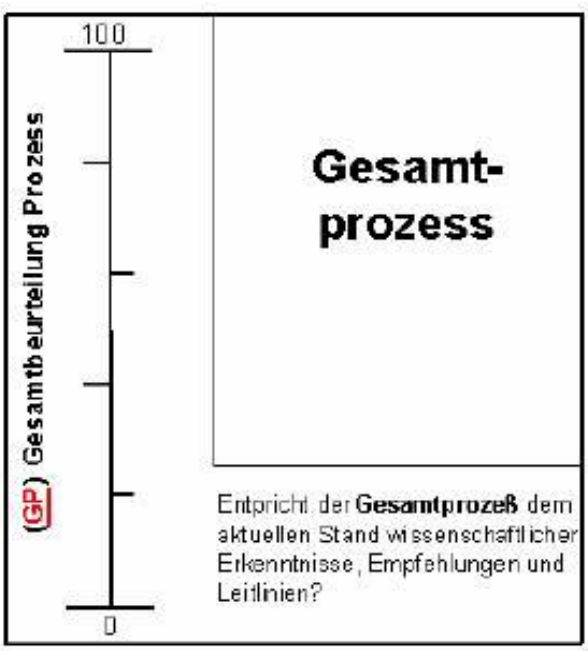

Abbildung 5. Visuelle Analogskala zur Beurteilung des Gesamtprozesses bezüglich der Entsprechung des aktuellen Standes wissenschaftlicher Erkenntnisse, Empfehlungen und Leitlinien

Am Ende der Checkliste hat der Gutachter anzugeben, ob eine Revisitation des Schlaflaboratoriums notwendig erscheint oder nicht. 
Abschließend stand dem Gutachter Platz für schriftliche Anmerkungen zu den vorliegenden Befunden und seine Beurteilung zur Verfügung.

$\mathrm{Zu}$ besserem Verständnis und klarer Definition für den Gutachter werden jedem Item im Manual nochmals Beurteilungshinweise nachgestellt. In den Beurteilungshinweisen wird nochmals erklärt, bei welcher Begebenheit genau das Item mit 'Empfehlung fehlt' codiert werden soll: „'Empfehlung fehlt' ist spezifischen diagnostischen Prozessen vorbehalten, bei denen nach Abschluss aller anderen Untersuchungen eine entsprechende weiterführende/weiter ausschließende Diagnostik zu erwarten gewesen wäre“.

Im Folgenden sollen exemplarisch für die Beurteilungsfelder Anamnese, Diagnostik, Therapie und Entlassungsbericht je ein Item und seine Beurteilungshinweise im Manual vorgestellt werden.

In dem Beurteilungsfeld Anamnese sollen die Gutachter u. a. das Item „Spezifische Anamnese in der Nacht" beurteilen. In den Beurteilungshinweisen im Manual wird dazu erläutert: „Aktuelle Beschwerden in der Nacht: Hier sollte nächtliche Problematik Erwähnung finden (z. B. Atemnot, Rhythmusstörungen, Zähneknirschen, Grübeln, etc.)“.

Grundsätzlich wird für die Beurteilung der allgemeine Hinweis gegeben, dass die Beurteilung als 'vorhanden' gilt, wenn dazu „für den jeweiligen Fall ausreichende Angaben im Entlassungsbricht existent sind“. Wurde „weder auf Vorbefunde verwiesen und finden sich keine eigenen Angaben“, so muss mit 'nicht vorhanden' beurteilt werden.

Im Manual heißt es für das Item „Komorbide Störungen erkannt/diagnostiziert“ des Beurteilungsfeldes Diagnostik: „Hier soll bewertet werden, ob komorbide Störungen anhand der Anamnese und gegebenenfalls aufgrund im Labor durchgeführter Untersuchungen erkannt worden sind [...]“. „Ist eine komorbide Störung erkannt worden“, so soll dies als 'vorhanden' gewertet werden, „ist diese aus den aufgeführten Befunden ersichtlich“, wird aber nicht aufgeführt, so wird mit 'nicht vorhanden' bewertet.

Bezüglich des Items „Medikamentöse Behandlungsmaßnahmen“ aus dem Beurteilungsfeld Diagnostik wird im Manual erwartet, dass „die eingeleitete medikamen- 
töse Therapie bereits in ihrer Auswirkung auf das schlafmedizinische Problem validiert wurde (Codierung als durchgeführt)“.

Das Beurteilungsfeld Entlassungsbericht enthält u. a. das Item „Stimmigkeit des therapeutischen Procedere“. Dazu steht im Manual: „Hier wird codiert, ob die erfolgte Therapie bzw. die Empfehlung hierzu sich schlüssig und sinnvoll aus den erhobenen Befunden ergibt und eine Therapiebegründung im Entlassungsbrief begründet wurde“.

Hervorzuheben ist, dass in diesem Beurteilungsfeld der Begriff 'angemessen' durch den „jeweiligen Gutachter spezifiziert“ werden muss, angelehnt an den „spezifischen Fall“. Zusätzlich erfolgt der Hinweis für das Kriterium 'fehlt' Es beinhaltet, dass eine Beurteilung der erfassten Parameter lediglich im Entlassungsbericht fehlt.

\subsection{Entscheidungskriterien für die Revisitation und das Fortbe- stehen der Akkreditierung}

Das Schlaflabor wird von den drei Gutachtern anhand der Patientenakte beurteilt und jeder Gutachter entscheidet jeweils, ob eine Revisitation des Schlaflabors notwendig erscheint. Tabelle 4 stellt die Kriterien, die über die Fortführung des Akkreditierungsstatus entscheiden, dar.

Tabelle 4. Überblick über die Kriterien zur Revisitationsentscheidung und die Fortführung des Akkreditierungsstatus

\begin{tabular}{|c|c|c|}
\hline $\begin{array}{c}\text { Revisitationsempfehlung } \\
\text { (insgesamt 3 Gutachter) }\end{array}$ & Revisitation & Akkreditienung \\
\hline 0 & nein & ja \\
1 & nein & ja \\
22 & ja & $\begin{array}{c}\text { nein- } \\
\text { erneute Früfungdurch } \\
\text { Eegehung des Schlaflabors }\end{array}$ \\
\hline
\end{tabular}

Das Schlafzentrum bleibt weiterhin akkreditiert, wenn keiner oder nur einer der Gutachter eine Revisitationsempfehlung ausspricht. Empfehlen mindestens zwei bzw. drei der Gutachter eine Revisitation, so wird zunächst das Fortbestehen der Akkreditierung nicht gewährt. Erst nach einer Revisitation, also einer Überprüfung 
durch Begehung durch drei Gutachter, wird erneut über die Akkreditierung entschieden.

\subsection{Datenverarbeitung und Analyse}

\subsubsection{Datenverarbeitung}

Der Analyse und Untersuchung der Fragestellung dienten ausschließlich die Daten der Checkliste qualitätsrelevanter Prozessmerkmale, die zuvor durch die Gutachter anhand von Patientenakten bewertet und ausgefüllt wurden.

Die Daten aus der durch die Gutachter bewerteten Checkliste wurden in eine Maske des Tabellenkalkulationsprogramm Excel 2007 (Windows) manuell eingegeben. Aus dem Stichtagsverfahren wurden 447 Patientenakten und aus dem Randomisierten Verfahren 354 Patientenakten eingefügt.

Der erste Schritt war die Erstellung eines übersichtlichen Kodierschemas. Für jede bewertete Patientenakte wurden die einzelnen bewerteten Items eingegeben. Dabei codierte die Zahl 1 für die vom Gutachter angekreuzte Antwortkategorie in der Checkliste und die Zahl 0 für jede frei gebliebene Antwortmöglichkeit. Abbildung 6 zeigt beispielhaft einen Ausschnitt der eingegebenen Daten.

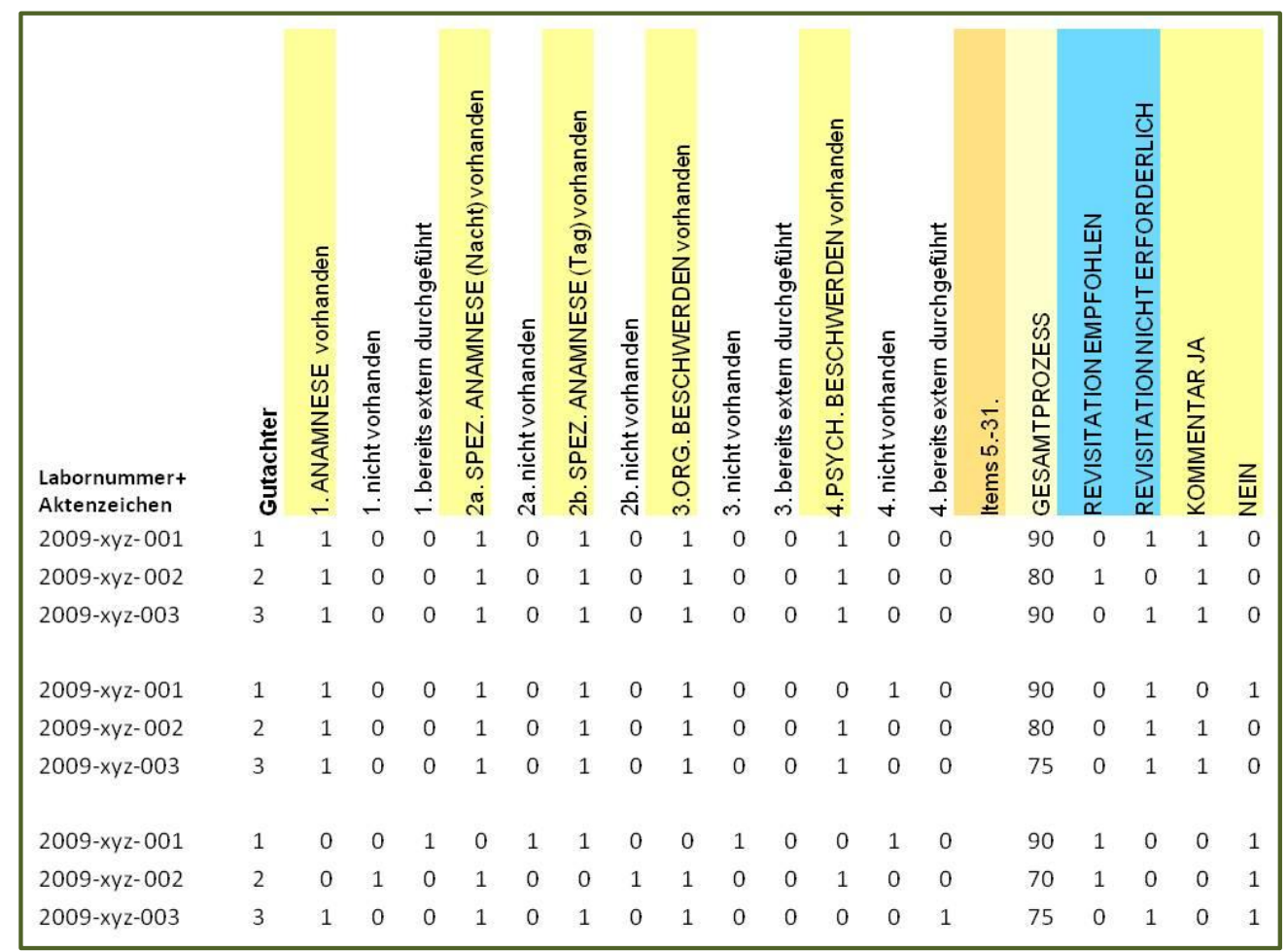

Abbildung 6. Übersicht des Kodierschemas im Tabellenkalkulationsprogramm Excel 
Wurden Items durch die Gutachter fälschlicherweise doppelt codiert, so wurde folgendermaßen gerechnet: Doppelte Codierung der Antwortmöglichkeit 'vorhanden' und 'bereits extern durchgeführt' wurde einzig als 'vorhanden' gewertet. Dies war ebenfalls der Fall bei der Mehrfachcodierung 'vorhanden' und 'Empfehlung ist erfolgt'. Die mehrmalige Codierung von 'nicht vorhanden' und 'Empfehlung fehlt' wurde mit 'nicht vorhanden' in die Rechnung aufgenommen. Die Festlegung basierte auf der Überlegung, dass in erster Linie entscheidend ist, ob ein Item vorhanden ist oder fehlt.

\subsubsection{Datenanalyse}

Zur statistischen und grafischen Datenanalyse wurde Excel 2007 verwendet und das Programm STATISTICA Version 9. Eine statistische Beratung wurde am 12.03.2010 bei Dr. Klaus Jung aus der Abteilung Medizinische Statistik des Zentrums für Informatik, Statistik und Epidemiologie Göttingen wahrgenommen.

Nur vollständige Patientenakten gingen in die Analyse ein. Es wurden demnach nur Schlaflabore mit drei begutachteten Patientenakten ausgewertet (Stichtagsverfahren 149, Randomisiertes Verfahren 118 Schlaflaboratorien).

Anhand der umfangreich erhobenen Daten wurde eine Häufigkeitsauszählung in Form absoluter und relativer Häufigkeiten vorgenommen. Auf diese Weise wurde verfahrensbezogen der Ausgang des Qualitätssicherungsverfahrens bezüglich der Revisitationsempfehlung bzw. der Weiterakkreditierung dargestellt.

Das Verhalten der Gutachter bezüglich der Revisitationsentscheidung wurde für jedes Verfahren einzeln untersucht. Um die Anonymisierung zu wahren, wurden den Gutachtern Zahlen zugeteilt. Es wurde die Anzahl abgegebener Revisitationsempfehlungen verfahrensbezogen gegenübergestellt und verglichen. In die Untersuchung wurden lediglich die 25 Gutachter, die an beiden Verfahren teilgenommen haben, einbezogen.

Des Weiteren wurden die durch die Gutachter bewerteten Items der Checkliste qualitätsrelevanter Prozessmerkmale einer Untersuchung unterzogen. Dazu wurde die absolute und relative Häufigkeit der Antwortkategorien für die einzelnen Prozessmerkmale bezogen auf das jeweilige Verfahren ausgezählt bzw. errechnet. Dabei wurde zwischen den Schlaflaboratorien mit Weiterakkreditierung und Schlaflaboratorien ohne Weiterakkreditierung unterschieden. Bei den Analysen 
und Zusammenhängen wurden fehlende Angaben ausgeschlossen. Demzufolge können in den Vergleichen jeweils leicht variierende Fallzahlen vorkommen.

Die am Ende der Checkliste zu beurteilende visuelle Analogskala (Abb. 5, S. 25), die angibt, ob der Gesamtprozess der Patientenakte dem aktuellen Stand wissenschaftlicher Erkenntnisse, Empfehlungen und Leitlinien entspricht, wurde auf folgende Weise dargestellt und analysiert. Angelehnt an die Auswertung des Qualitätssicherungsprogramms in der pneumologischen Praxis (Fischer et al. 2006) wurde für einzelne Werte eine Wertung zugeordnet. Dazu wurde der Gesamtprozess in die Gruppen von $\geq 80=$ "gute bis sehr gute" Prozessqualität, 60-80 = "durchschnittliche“ Prozessqualität und $\leq 60=$,schwach ausgeprägte bis defizitäre" Prozessqualität unterteilt (Fischer et al. 2006, S. 488).

Zur Signifikanzbeurteilung bivariater Zusammenhänge kategorialer Variablen wurde der $\mathrm{Chi}^{2}$-Vierfeldertest nach Pearson verwendet. Das Signifikanzniveau wurde mit $\alpha=0,05$ und damit $\mathrm{Chi}^{2}=>3,841$ festgelegt. Nachgewiesene Unterschiede zwischen zwei Variablen wurden ab einer Irrtumswahrscheinlichkeit von $p \leq 0,05$ als signifikant gewertet. Weiterhin wurde das $\alpha$-Niveau mit der Durchführung einer Bonferroni-Korrektur angepasst $(\alpha=0,0014)$.

Mittels des $\mathrm{Chi}^{2}$-Tests wurde zum einen die Abhängigkeit der Entscheidung der Akkreditierung in Bezug auf das angewendete Verfahren bestimmt (Bezug Schlaflaboratorien). Zum anderen wurde verfahrensbezogen die signifikante Abhängigkeit eines 'vorhandenen' bzw. 'fehlenden' Items auf die Revisitationsentscheidung des Gutachters untersucht (Datenbezug Patientenakten). Zur Klärung dieser Abhängigkeit wurde des Weiteren die vom Gutachter als 'vorhanden' oder 'nicht vorhanden' beurteilten Items der Revisitationsentscheidung gegenübergestellt und ihre Häufigkeitsverteilung betrachtet. Auf diese Weise wird ersichtlich, ob Items mit negativer Beurteilung ('nicht vorhanden', 'Empfehlung fehlt') besonders häufig mit einer Revisitationsempfehlung assoziiert waren. Umgekehrt lässt sich eine Aussage darüber treffen, ob eine positive Beurteilung ('vorhanden', 'Empfehlung ist erfolgt') seltener mit einer Revisitationsempfehlung einherging. Die Antwortmöglichkeit 'entfällt' ließ sich keiner Kategorie sinnvoll zuordnen und ging somit nicht in die Rechnung mit ein. 
Aufgrund der Datenstruktur (kategorial) konnte eine Faktorenanalyse, die aus empirischen Beobachtungen vieler verschiedener Variablen auf wenige zugrunde liegende latente Variablen zu schließen hilft, nicht durchgeführt werden.

Aufgrund der umfangreichen Daten (801 Patientenakten) wurde zur Auszählung und Analyse zusätzlich das Programm STATISTICA zu Hilfe genommen.

\subsection{Datenschutz und Ethikvotum}

Die Daten der Patienten wurden in der jeweiligen Patientenakte vor dem Verschicken an die Zentralstelle der DGSM pseudonymisiert. Auf der Checkliste erschienen nur das Geburtsdatum und die Initialen des Patienten, die ausschließlich der Zuordnung der Patientenakten zum jeweiligen Schlaflaboratorium dienten. Da die Auswertung ausschließlich auf den Daten der Checkliste basierte und nicht auf den Patientenakten direkt, war kein Ethikvotum notwendig (siehe Anhang 8.6). 


\section{Ergebnisse}

\subsection{Ergebnisse zum organisatorischen und zeitlichen Verlauf}

Einige wichtige Daten zum Ablauf der Qualitätssicherung 2009/10 werden in Tabelle 5 dargestellt. Im Rahmen der Qualitätssicherung nehmen insgesamt 88,2 \% der angeschriebenen Schlaflaboratorien am Stichtagsverfahren teil. Im Randomisierten Verfahren liegt die Teilnahme bei 72,7 \% der angeschriebenen Schlafzentren. Die Gründe für die Nichtteilnahme lagen u. a. an unvollständigen Akten oder fehlendem Interesse am Fortbestehen des Akkreditierungsstatus.

Während die gesamten Daten der Schlaflaboratorien, die am Stichtagsverfahren teilgenommen haben, in die Analyse eingegangen sind, fanden nur 92,2 \% der Schlaflaboratorien des Randomisierten Verfahrens in der Untersuchung Berücksichtigung. Dies beruht auf der Tatsache, dass ausschließlich vollständige Auswertungen betrachtet werden sollten.

Die Dauer des Randomisierten Verfahrens ist mit 16 Monaten doppelt so lang wie die Durchführung des Stichtagsverfahrens.

Tabelle 5. Wichtige Daten zum Ablauf des Stichtagsverfahrens und des Randomisierten Verfahrens im Vergleich

${ }^{*} \mathrm{SL}=$ Schlaflabor, ${ }^{*} \mathrm{QS}=$ Qualitätssicherung

\begin{tabular}{|l|c|c|}
\hline \multicolumn{2}{c}{ Stichtagsverfahren } & $\begin{array}{c}\text { Randomisiertes } \\
\text { Verfahren }\end{array}$ \\
\hline Angeschriebene SL & 169 & 176 \\
\hline Teilnehmende SL & $149(88,2 \%)$ & $128(72,7 \%)$ \\
\hline $\begin{array}{l}\text { In die Analyse der Arbeit } \\
\text { eingegangene SL }\end{array}$ & $149(100 \%)$ & $118(92,2 \%)$ \\
\hline Anzahl Gutachter & 30 & 29 \\
\hline & & Oktober 2009 \\
\hline Beginn der QS & Januar 2009 & Februar 2011 \\
\hline Ende der QS & August 2009 & 16 Monate \\
\hline Insgesamt Dauer & \multicolumn{2}{c|}{} \\
\hline
\end{tabular}




\subsection{Ergebnis der Weiterakkreditierung und die Abhängigkeit des jeweiligen Verfahrens}

Im Stichtagsverfahren behalten $91 \%(\mathrm{~N}=135)$ der teilnehmenden Schlaflaboratorien weiterhin den Akkreditierungsstatus. $9 \%(N=14)$ der Labore verlieren den Status der Akkreditierung durch die DGSM und müssen sich einer erneuten Überprüfung ihrer Leistung (Revisitation) unterziehen. Im Randomisierten Verfahren bleiben $92 \%(N=109)$ der teilnehmenden Schlafzentren weiterhin akkreditiert. $8 \%(N=9)$ verlieren vorerst den Akkreditierungsstatus und werden sich ebenfalls einer Revisitation unterziehen müssen. Abbildung 7 zeigt das nahezu identische Ergebnis.

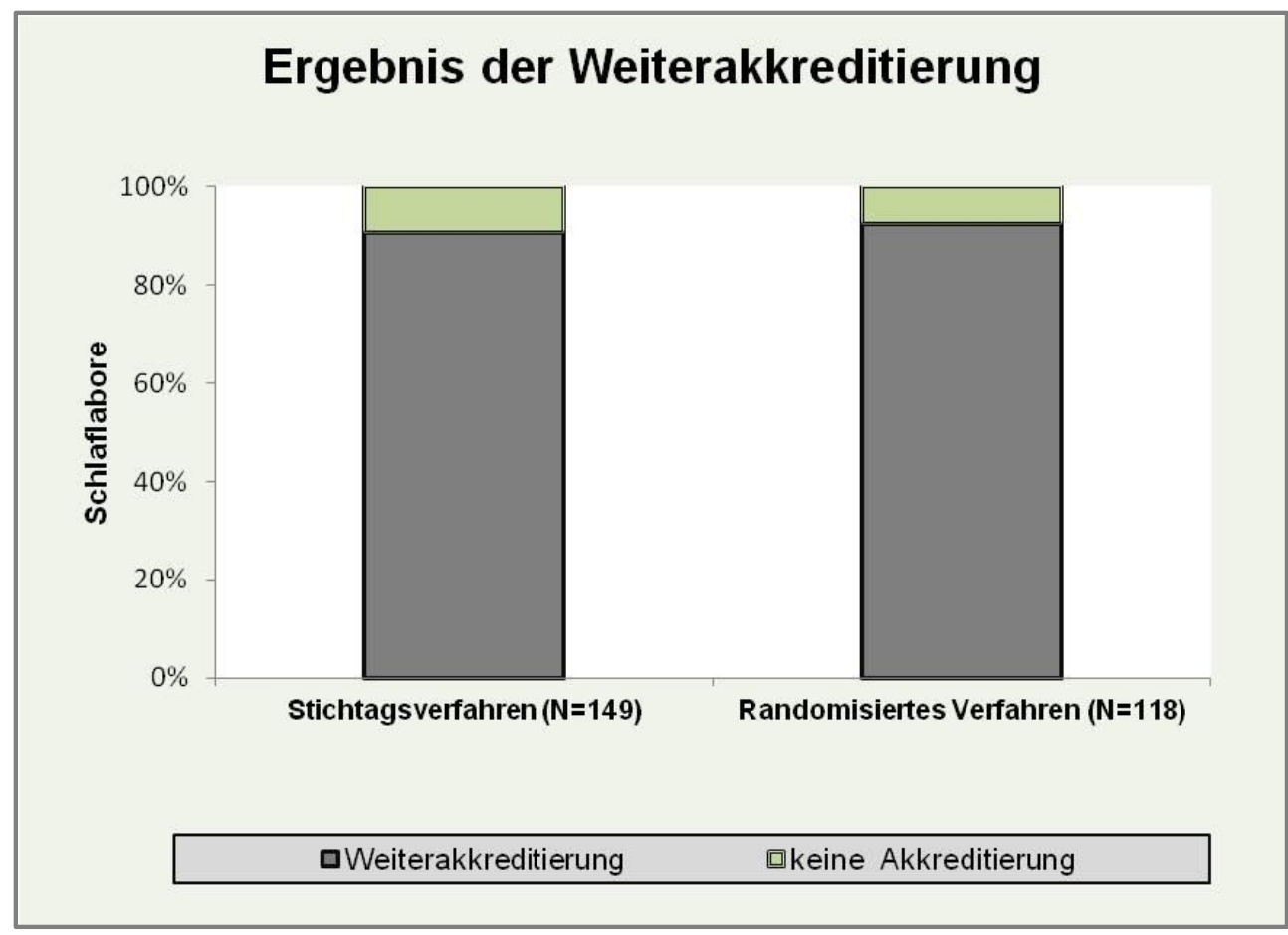

\section{Abbildung 7. Ergebnis der Weiterakkreditierung der am Qualitätssicherungs- programm teilnehmenden Schlaflaboratorien im Vergleich des Stichtags- bzw. Randomisierten Verfahrens}

Es konnte gezeigt werden, dass das methodische Vorgehen keinen Einfluss auf das Ergebnis bezüglich einer Weiterakkreditierung des Schlaflabors nimmt. Unabhängig von der Methode zur Aktenauswahl, zeigt sich ein sehr ähnliches Ergebnis bezüglich der Revisitationsempfehlungen (Abb. 8) und der Anzahl der weiterhin akkreditierten Schlafzentren (Abb. 7).

Der Chi' ${ }^{2}$-Test stützt dieses Ergebnis (Tab. 6). Der Chi ${ }^{2}$-Wert liegt mit 0,262 unter 3,81 und der berechnete $p$-Wert liegt bei 0,609. Es wird offensichtlich, dass kein 
signifikanter Unterschied zwischen den beiden methodischen Verfahren im Hinblick auf die Weiterakkreditierung nachgewiesen werden konnte.

Tabelle 6. Vergleich des Stichtagsverfahrens und des Randomisierten Verfahrens im Hinblick auf die Weiterakkreditierung (Chi ${ }^{2}$-Test)

\begin{tabular}{ccc}
\hline & Weiterakkreditierung & Keine Akkreditierung \\
\hline Stichtagsverfahren $(\mathrm{N}=149)$ & $135(91 \%)$ & $14(9 \%)$ \\
\hline Randomisiertes Verfahren $(\mathrm{N}=118)$ & $109(92 \%)$ & $9(8 \%)$ \\
\hline Chi $^{2}$-Wert & 0,262 & \\
\hline p-Wert & 0,609 & \\
\hline
\end{tabular}

\subsection{Ergebnis der Revisitationsempfehlungen der Gutachter}

\subsubsection{Stichtagsverfahren}

$71,1 \%$ der Schlaflaboratorien $(N=106)$ erhalten keine Empfehlung zur Revisitation. In 19,5\% der Laboratorien ( $\mathrm{N}=29)$ spricht sich einer der drei Gutachter für eine Revisitation aus. Bei $7,4 \%$ der Schlafzentren $(\mathrm{N}=11)$ befürworten zwei der drei Gutachter eine Revisitation. $2 \%$ der Schlaflaboratorien ( $N=3$ ) erhalten einstimmig eine Empfehlung zur Revisitation (vgl. Abb. 8).

Bei der Betrachtung der drei pro Schlaflabor bewerteten Patientenakten ergibt sich, dass von insgesamt 447 Patientenakten $13,4 \%$ (60) eine Revisitationsempfehlung vom Gutachter ausgesprochen bekommen haben.

\subsubsection{Randomisiertes Verfahren}

$68,6 \%$ der Schlaflaboratorien $(\mathrm{N}=81)$ erhalten keine Revisitationsempfehlung. In $23,7 \%$ der Laboratorien ( $\mathrm{N}=28$ ) empfiehlt einer der Gutachter die Revisitation. In $5,9 \%$ der Schlafzentren ( $N=7)$ sprechen sich zwei Gutachter für eine Revisitation aus. $1,8 \%$ der Schlaflaboratorien $(N=2)$ erhalten einstimmig (also 3) Revisitationsempfehlungen (vgl. Abb. 8).

Von den 354 untersuchten Patientenakten werden dementsprechend 13,6 \% (48) mit einer Empfehlung zur Revisitation versehen. 


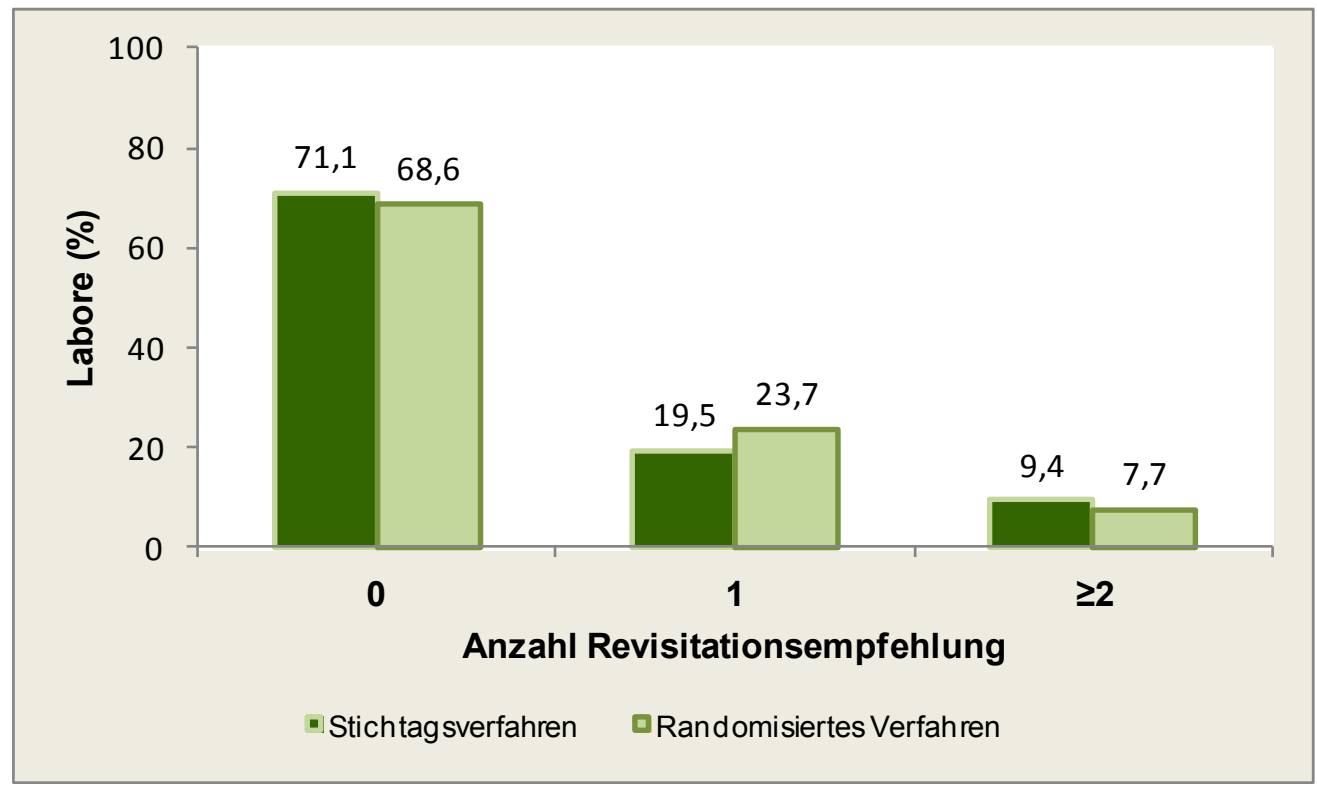

Abbildung 8. Anteile der Schlaflaboratorien mit keiner/einer/mehr als zwei Revisitationsempfehlungen im Vergleich zwischen Stichtagsverfahren und Randomisiertem Verfahren

\subsection{Verhalten der Gutachter bezüglich der Revisitationsentscheidung im Vergleich der beiden methodi- schen Verfahren}

Die Revisitationsentscheidung der Gutachter in beiden Verfahren wurde ebenfalls einer Analyse unterzogen. Die Fragestellung war, ob sich Gutachter bestimmen lassen, die tendenziell mehr Revisitationsempfehlungen aussprechen und damit strenger erscheinen als andere.

Im Stichtagsverfahren nahmen insgesamt 29 Gutachter teil und bewerteten jeweils zwischen 13 und 17 Patientenakten. Im Randomisierten Verfahren bewerteten insgesamt 30 Gutachter anhand 8-16 Patientenakten. 25 Gutachter nahmen an beiden methodischen Verfahren teil. Die übrigen mussten aufgrund fehlender Compliance, Umzug oder sonstiger Umstände ausgetauscht werden.

Tabelle 7 zeigt einen Vergleich der Häufigkeit der Revisitationsempfehlung der 25 an beiden Verfahren teilnehmenden Gutachter.

Mehr als die Hälfte der Gutachter (56 \%) empfehlen in 10 bis $20 \%$ der untersuchten Patientenakten eine Revisitation. Zwei Gutachter empfehlen in mehr als $30 \%$ ihrer Patientenakten eine Revisitation (vgl. Tab. 7 Gutachter 22 und 25).

Wiederum zwei Gutachter verzichten in beiden Durchgängen gänzlich auf Revisitationsempfehlungen (siehe Tab. 7 Gutachter 8 und 15). 
Tabelle 7. Übersicht über die Häufigkeit der Revisitationsempfehlungen der 25 an beiden Verfahren teilnehmenden Gutachter

${ }^{*} \mathrm{SV}=$ Stichtagsverfahren, *RV = Randomisiertes Verfahren

\begin{tabular}{|c|c|c|c|}
\hline $\begin{array}{l}\text { Gutach- } \\
\text { ter }\end{array}$ & $\begin{array}{c}\text { Revisitationsempfehlung } \\
\text { gesamt } \%\end{array}$ & $\begin{array}{c}\text { Revisitationsempfehlung } \\
\text { SV \% }\end{array}$ & $\begin{array}{c}\text { Revisitationsempfehlung } \\
\text { RV \% }\end{array}$ \\
\hline 1. & $12,7(\mathrm{~N}=23)$ & $15,4(\mathrm{~N}=13)$ & $10(\mathrm{~N}=10)$ \\
\hline 2. & $10,1(\mathrm{~N}=29)$ & $12,5(\mathrm{~N}=16)$ & $7,7(\mathrm{~N}=13)$ \\
\hline 3. & $8,2(N=27)$ & $6,3(N=16)$ & $10(N=11)$ \\
\hline 4. & $11,3(\mathrm{~N}=27)$ & $12,5(\mathrm{~N}=16)$ & $10(\mathrm{~N}=11)$ \\
\hline 5. & $3,6(N=29)$ & $0(N=15)$ & $7,1(\mathrm{~N}=14)$ \\
\hline 6. & $13,4(\mathrm{~N}=25)$ & $6,7(\mathrm{~N}=15)$ & $20(N=10)$ \\
\hline 7. & $10(N=23)$ & $0(N=13)$ & $20(N=10)$ \\
\hline 8. & $0(\mathrm{~N}=22)$ & $0(\mathrm{~N}=14)$ & $0(\mathrm{~N}=8)$ \\
\hline 9. & $20,2(N=29)$ & $33,3(\mathrm{~N}=15)$ & $7,1(\mathrm{~N}=14)$ \\
\hline 10. & $3,6(N=26)$ & $7,1(\mathrm{~N}=14)$ & $0(\mathrm{~N}=12)$ \\
\hline 11. & $22,5(\mathrm{~N}=26)$ & $26,7(\mathrm{~N}=15)$ & $18,2(\mathrm{~N}=11)$ \\
\hline 12. & $18,2(\mathrm{~N}=28)$ & $13,3(\mathrm{~N}=15)$ & $23,1(\mathrm{~N}=13)$ \\
\hline 13. & $5(\mathrm{~N}=24)$ & $0(\mathrm{~N}=14)$ & $10(\mathrm{~N}=10)$ \\
\hline 14. & $13,3(N=29)$ & $18,8(\mathrm{~N}=16)$ & $7,7(\mathrm{~N}=13)$ \\
\hline 15. & $0(\mathrm{~N}=27)$ & $0(\mathrm{~N}=15)$ & $0(\mathrm{~N}=12)$ \\
\hline 16. & $17,7(\mathrm{~N}=28)$ & $35,3(\mathrm{~N}=17)$ & $0(N=11)$ \\
\hline 17. & $12,5(\mathrm{~N}=17)$ & $0(N=15)$ & $25(\mathrm{~N}=12)$ \\
\hline 18. & $10,5(\mathrm{~N}=28)$ & $13,3(\mathrm{~N}=15)$ & $7,7(\mathrm{~N}=13)$ \\
\hline 19. & $11,5(\mathrm{~N}=28)$ & $6,3(N=16)$ & $16,7(\mathrm{~N}=12)$ \\
\hline 20. & $24,2(N=30)$ & $17,6(\mathrm{~N}=17)$ & $30,8(\mathrm{~N}=13)$ \\
\hline 21. & $15,7(\mathrm{~N}=30)$ & $31,3(\mathrm{~N}=16)$ & $0(N=14)$ \\
\hline 22. & $34(\mathrm{~N}=23)$ & $25(\mathrm{~N}=16)$ & $42,9(\mathrm{~N}=7)$ \\
\hline 23. & $3,4(N=25)$ & $6,7(N=15)$ & $0(\mathrm{~N}=10)$ \\
\hline 24. & $9,8(N=30)$ & $12,5(\mathrm{~N}=16)$ & $7,1(N=14)$ \\
\hline 25. & $35(\mathrm{~N}=25)$ & $20(N=15)$ & $50(\mathrm{~N}=10)$ \\
\hline
\end{tabular}

Stellt man den Anteil der Revisitationsempfehlungen des einzelnen Gutachters der beiden Verfahren gegenüber, lässt sich kein Zusammenhang zwischen der Art des Verfahrens beziehungsweise der Wahl des Gutachters ableiten. Gutachter, die im Stichtagsverfahren viele Revisitationsempfehlungen abgegeben haben, tun dies im nächsten Verfahren nicht zwangsläufig. 
Besonders deutlich lässt sich dies am Beispiel der Gutachter 16 und 21 (siehe Tab. 7) nachweisen. Diese geben im Stichtagsverfahren die meisten Revisitationsempfehlungen ab (35 bzw. 31 \%). Im Randomisierten Verfahren wurde keine einzige Revisitationsempfehlung von innen ausgesprochen.

\subsection{Bewertungsergebnisse - Itemanalyse}

\subsubsection{Schlaflaboratorien mit Weiterakkreditierung - verfahrens- bezogen}

Im Folgenden soll zunächst das gesamte Ergebnis der Qualitätssicherung der weiterhin akkreditierten Schlaflaboratorien vorgestellt werden. Dazu wurde der Erfüllungsgrad der untersuchten Prozessmerkmale (Items) in den fünf Beurteilungsebenen Anamnese, Diagnostik, Diagnosesicherung, Therapie und Entlassungsbericht geprüft. Die Ergebnisse für beide Verfahren sind in den Abbildungen 9 bis 12 und den Tabellen 8 und 9 angegeben. Die einzelnen Zahlenwerte können im Anhang 8.7 nachvollzogen werden. Aus den angegebenen Ergebnissen lässt sich die allseitige Prozessqualität in den weiterhin von der DGSM akkreditierten Schlaflaboratorien ableiten.

Im Beurteilungsfeld Anamnese lassen sich die meisten fehlenden Items finden (siehe Abb. 9). Es fällt auf, dass besonders häufig sowohl im Stichtagsverfahren als auch im Randomisierten Verfahren die Angaben zu „psychischen“ (SV = 32,1 $\%, \mathrm{RV}=26 \%$ ) und „vegetativen ( $\mathrm{SV}=26,2 \%, \mathrm{RV}=25,7 \%$ ) Beschwerden“, „psychosozialen Belastungen“ (SV = $36 \%$, RV = 31,5\%) und „Risikofaktoren bzw. Suchtanamnese" (SV = 27,9 \%, RV = $22 \%$ ) für den Gutachter nicht in den Patientenakten zu erfassen waren (vgl. Abb. 9).

Ferner wird offensichtlich, dass im Randomisierten Verfahren die meisten Items für den Gutachter häufiger als 'vorhanden' erfasst wurden (Tab.9). Während im Stichtagsverfahren in 13,3\% der Patientenakten Angaben zur "Allgemeinen Anamnese" 'nicht vorhanden' waren, waren es im Randomisierten Verfahren 7,6 $\%$ der Fälle. Die Spezifische Anamnese am Tag bzw. in der Nacht war im Stichtagsverfahren für die Gutachter in 9,4\% (Nacht) und 8,4 \% (Tag) nicht ersichtlich.

Im Randomisierten Verfahren bewerteten die Gutachter die Angaben in 5,5\% (Nacht) und 3,5 \% (Tag) der Fälle als 'nicht vorhanden'. Insgesamt lässt sich fest- 
stellen, dass im überwiegenden Teil der Schlafzentren die anamnestischen Angaben für den Gutachter ersichtlich waren.

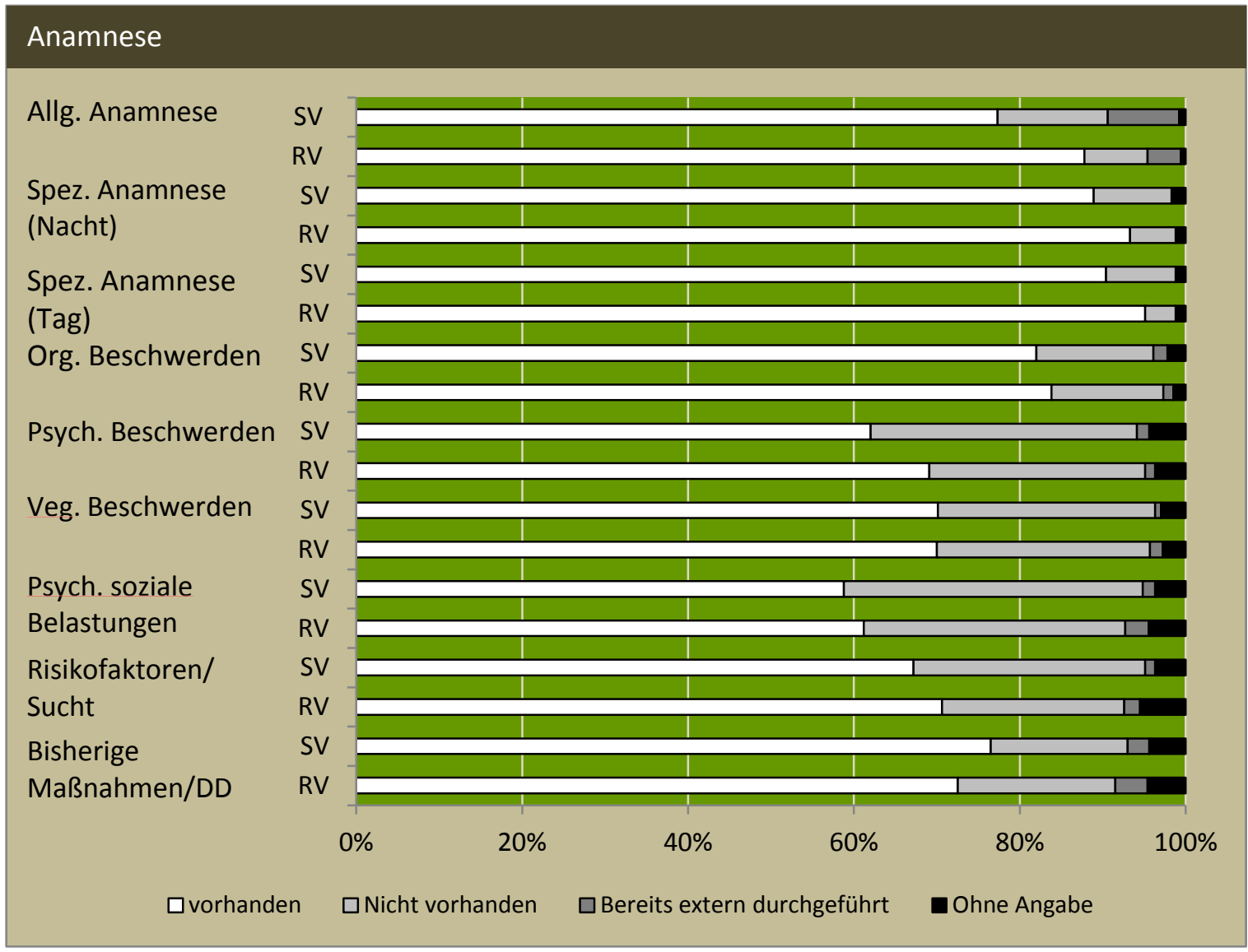

\section{Abbildung 9. Häufigkeitsverteilung der erfassten Prozessmerkmale aus dem Beurteilungsfeld Anamnese für die weiterhin akkreditierten Schlaflabore}

${ }^{*} \mathrm{SV}=$ Stichtagsverfahren, ${ }^{*} \mathrm{RV}=$ Randomisiertes Verfahren

Die Items aus dem Beurteilungsfeld Diagnostik wurden im Randomisierten Verfahren im Vergleich zum Stichtagsverfahren ebenfalls häufiger als 'vorhanden' vom Gutachter erfasst. Es lässt sich feststellen, dass alle die Diagnostik betreffenden Items im Stichtagsverfahren in mindestens $10 \%$ fehlten.

Die Items, die laut Gutachter am häufigsten 'nicht vorhanden' waren, waren sowohl im Stichtagsverfahren als auch im Randomisierten Verfahren die Angaben zur Durchführung der „Leistungs- und Vigilanzuntersuchung“ bzw. eines „klinischen Labors“ (Abb. 10). Im Stichtagsverfahren wurden diese in 37,8 \% („Leistungs- und Vigilanzuntersuchung“) und 27,7 \% („klinisches Labor“) als 'nicht vorhanden' bewertet. Im Randomisierten Verfahren wurden in 40,1 \% der Fälle keine 
Angaben zur „Leistungs- und Vigilanzuntersuchung“ angegeben und in $26 \%$ 'fehlten' Angaben zur Durchführung eines „klinischen Labors“.

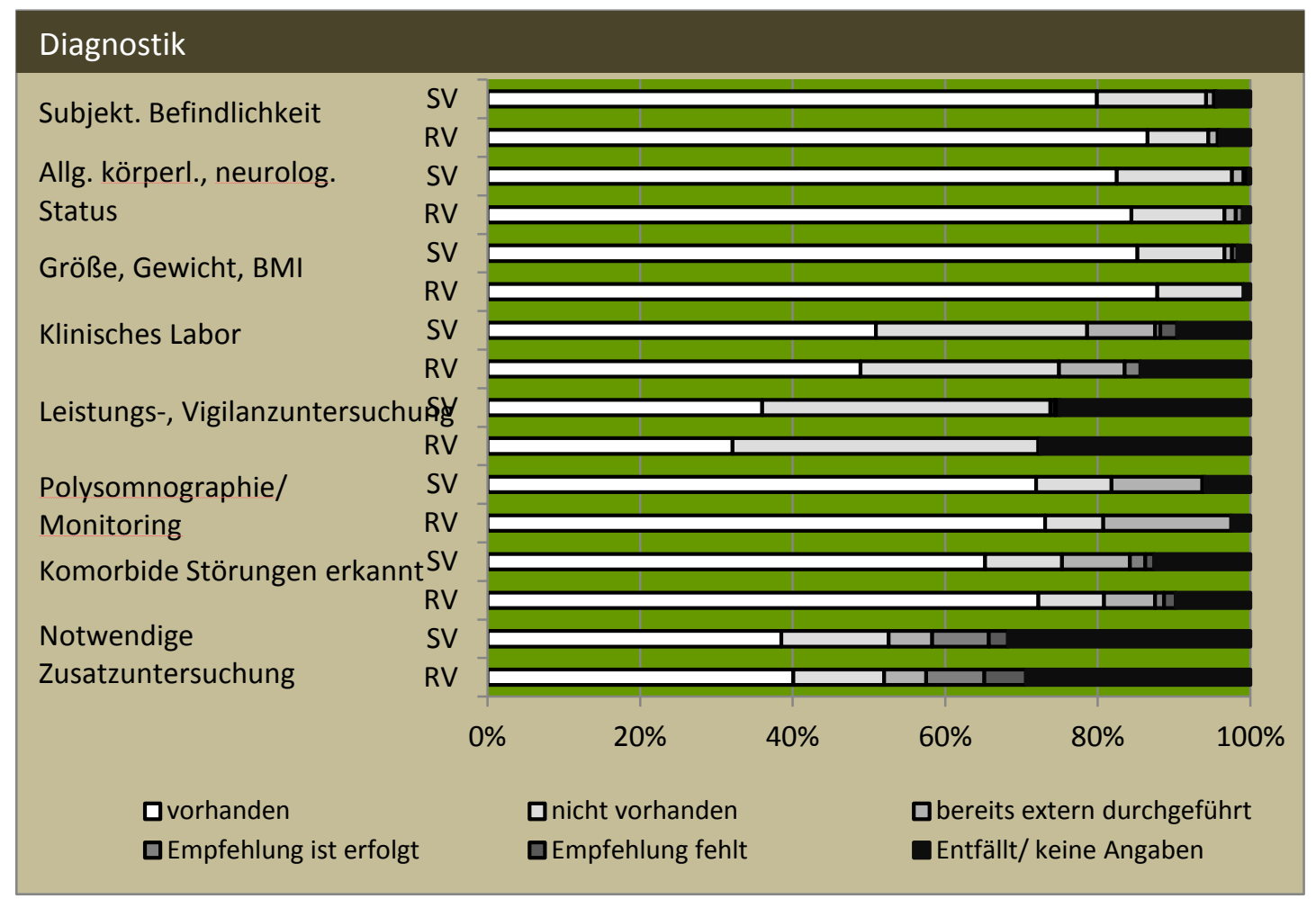

\section{Abbildung 10. Häufigkeitsverteilung der durch die Gutachter erfassten Items im Beurteilungsfeld Diagnostik in den weiterhin akkreditierten Schlaflaboratorien}

${ }^{*} \mathrm{SV}=$ Stichtagsverfahren, ${ }^{*} \mathrm{RV}=$ Randomisiertes Verfahren

Patientenorientierte Items wie die „Subjektive Befindlichkeit“ oder der „Allgemein körperliche, neurologisch- psychiatrische Status“ konnten im Randomisierten Verfahren etwas häufiger als 'vorhanden' erfasst werden. Dennoch lag der als 'nicht vorhanden' erfasste Anteil der „Subjektiven Befindlichkeit“ im Randomisierten Verfahren bei $8 \%(S V=14,3 \%)$ und der „Allgemein körperliche, neuropsychologische Status“' 'fehlte' in 12,2 \% (SV = 15,1 \%) der Fälle.

„Komorbide Störungen“ wurden im Stichtagsverfahren in 10,1 \% als „nicht erkannt“ angegeben, im Randomisierten Verfahren war das in 8,6 \% der Patientenakten der Fall.

Aus dem Beurteilungsfeld Diagnosesicherung war das Item 17 "Aktuell im Schlaflabor durchgeführte Untersuchungen" aufgrund mangelnder Beurteilung durch die Gutachter nicht eindeutig auswertbar und damit nicht aussagekräftig. Die Beurtei- 
lung der Angemessenheit (adäquat/nicht adäquat) der durchgeführten Untersuchungen wurde im Stichtagsverfahren nur in $60 \%(\mathrm{~N}=787)$ der untersuchten Akten von den Gutachtern vorgenommen. Im Randomisierten Verfahren wurde die Angemessenheit der durchgeführten Untersuchungen in nur 68,8 \% ( $N=644)$ beurteilt, d.h. beinahe $1 / 3$ wurde nicht bewertet.

Das angefertigte Nachtprotokoll war in 63,5 \% (Stichtagsverfahren) der Patientenakten vollständig und in $6 \%$ nicht zu verwerten. Im Randomisierten Verfahren erfüllten $67 \%$ der Nachtprotokolle die Vollständigkeit. $6 \%$ der Nachtprotokolle waren nicht zu verwerten.

Die Dokumentation von Messgrößen der durchgeführten Untersuchungen wurden nahezu „vollständig“ bzw. „teilweise“ im Entlassungsbericht vorgefunden (Tab. 8). Nur die Angaben zur Lagebestimmung bzw. Körperposition des behandelten Patienten 'fehlten' im Stichtagsverfahren in $19 \%$ und im Randomisierten Verfahren in $15,6 \%$ der Patientenakten.

Tabelle 8. Häufigkeitsverteilung der erfassten Messgrößen in den Patientenakten der weiterhin akkreditierten Schlafzentren

${ }^{*} \mathrm{SV}=$ Stichtagsverfahren, ${ }^{*} \mathrm{RV}=$ Randomisiertes Verfahren

\begin{tabular}{|c|c|cccc|}
\hline Messgrößen & Verfahren & vollständig (\%) & teilweise (\%) & fehlt (\%) & $\begin{array}{c}\text { entfällt/ohne } \\
\text { Angabe (\%) }\end{array}$ \\
\hline Globale Maße & SV & 83 & 13,6 & 1,7 & 1,7 \\
\hline & RV & 85,3 & 10,1 & 3,1 & 1,5 \\
\hline Latenzen & SV & 84,4 & 7,4 & 5,7 & 2,5 \\
\hline & RV & 81,3 & 10,4 & 6,1 & 2,2 \\
\hline Prozentuale Anteile & SV & 88,6 & 5,7 & 4,7 & 1 \\
\hline & RV & 89,6 & 5,5 & 3,1 & 1,8 \\
\hline Atmung, Sättigung, & SV & 88,1 & 10,9 & & 1,5 \\
\hline Schnarchen & RV & 86,9 & 10,7 & 0,9 & 4,3 \\
\hline & SV & 68,1 & 8,6 & 19 & 3 \\
\hline Lage & RV & 73,1 & 8,3 & 15,6 & 3,7 \\
\hline Beinbewegungen & SV & 78,3 & 11,6 & 6,4 & 3,9 \\
\hline & RV & 82,3 & 8,3 & 5,5 & 3,4 \\
\hline Arousal & SV & 76,5 & 13,3 & 7,2 & 4,9 \\
\hline & RV & 82 & 10,7 & & \\
\hline
\end{tabular}

Die Häufigkeitsverteilung der erfassten Items, die therapeutische Maßnahmen betreffen, zeigt, dass häufig die Bewertung der „Psychologischen“ und „Medikamentösen Therapie“" 'entfällt' (vgl. Abb. 11). Im Stichtagsverfahren 'entfällt' die Beurteilung des Items „Psychologische Behandlungsmaßnahmen“ in 59,7 \% der Fälle 
(RV $=68,8 \%$ der Fälle) und des Items "Medikamentöse Therapie" in 61,2 \% der Fälle (RV $=73,4 \%$ der Fälle).

Im Durchschnitt 'fehlten' im Randomisierten Verfahren in $9 \%$ der Patientenakten therapeutisch notwendige Maßnahmen (siehe Abb. 11): „Medikamentöse Therapie" 7,0 \% (SV = 6,7 \%), „Psychotherapeutische Therapie“ 11,3\% (SV = 12,8\%) und „Allgemeine Therapie“ in 8,3 \% der Fälle (SV = 10,4 \%).

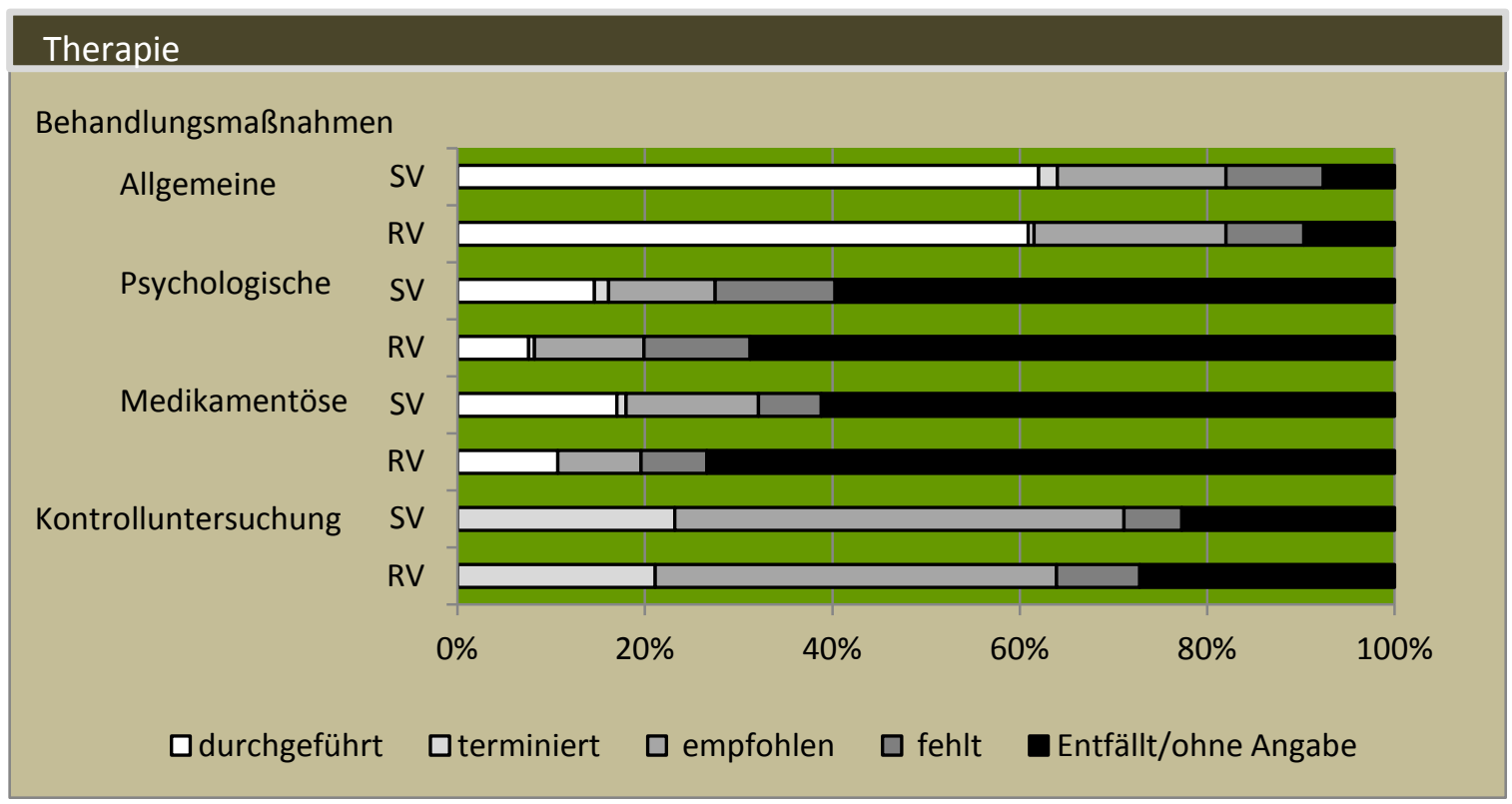

\section{Abbildung 11. Häufigkeitsverteilung der erfassten Items aus dem Beurtei- lungsfeld Therapie für die weiterhin akkreditierten Schlaflaborato-} rien.

${ }^{*} \mathrm{SV}=$ Stichtagsverfahren, ${ }^{*} \mathrm{RV}=$ Randomisiertes Verfahren

Die „Zusammenfassende Beurteilung“ der „Schlaf-, Atmungsparameter und Beinbewegungen" aus dem Beurteilungsfeld Entlassungsbericht war im Ganzen zu einem hohen Anteil 'angemessen' bzw. 'teilweise angemessen' (siehe Tab. 9). Auffällig war in beiden Verfahren ein hoher Anteil 'fehlender' ( $S V=9,1 \%, R V=5,8 \%$ ) bzw. 'nicht angemessener' Beurteilung der Beinbewegungen ( $S V=5,4 \%, R V=$ $5,2 \%)$. 
Tabelle 9. Häufigkeitsverteilung der Bewertung der „Zusammenfassenden Beurteilung“ der „Schlaf-, Atmungsparameter und Beinbewegungen“ im Entlassungsbericht der weiterhin akkreditierten Schlafzentren

${ }^{*} \mathrm{SV}=$ Stichtagsverfahren, ${ }^{*} \mathrm{RV}=$ Randomisiertes Verfahren

\begin{tabular}{|l|c|ccccc|}
\hline \multicolumn{1}{|c|}{ Item } & Verfahren & $\begin{array}{c}\text { angemessen } \\
(\%)\end{array}$ & $\begin{array}{c}\text { teilweise angemessen } \\
(\%)\end{array}$ & $\begin{array}{c}\text { nicht angemessen } \\
(\%)\end{array}$ & $\begin{array}{c}\text { fehlt } \\
(\%)\end{array}$ & $\begin{array}{c}\text { entfällt/ohne Angabe } \\
(\%)\end{array}$ \\
\hline Verwendungder & SV & 77,8 & 18 & 2 & 1,7 & 0,5 \\
Standardnomenklatur & RV & 78,6 & 17,7 & 1,2 & 1,2 & 1,3 \\
Zusammenfassende & & & & & & \\
Bewertung & & & & & & \\
a) Schlafparameter & SV & 77,5 & 13,1 & 4 & 2 & 3,4 \\
& RV & 75,2 & 14,4 & 5,2 & 3,4 & 1,8 \\
\multicolumn{1}{c|}{ b) Atmung } & SV & 85,4 & 10,6 & 1,5 & 1,5 & 1 \\
& RV & 84,7 & 13,5 & 0,3 & 0,3 & 1,2 \\
\multicolumn{1}{c|}{ c) Beinbewegung } & SV & 66,4 & 10,4 & 5,4 & 9,1 & 8,7 \\
& RV & 68,8 & 10,1 & 5,2 & 5,8 & 10,1 \\
\hline
\end{tabular}

Die Beurteilung der „Stimmigkeit des therapeutischen und diagnostischen Procedere" erschien insgesamt bei einem hohen Anteil der Schlaflaboratorien als 'angemessen' bzw. 'teilweise angemessen' (siehe Abb. 12).

Im Stichtagsverfahren wurde die "Stimmigkeit des diagnostischen Procedere" in 93,3 \% der Fälle als 'angemessen' beurteilt, davon 20,2 \% der Fälle nur 'teilweise angemessen'. In 4,1 \% der Patientenakten fand kein 'angemessenes' diagnostisches Procedere statt.

Im Randomisierten Verfahren wurde in 95,7 \% der Patientenakten die „Stimmigkeit des diagnostischen Vorgehens" als 'angemessen', davon 20,1\% als 'teilweise angemessen' bewertet. In 3,7 \% erschloss sich dem Gutachter kein 'angemessenes' diagnostisches Vorgehen.

Die "Stimmigkeit des therapeutischen Procedere" wurde in 90,6 \% der Patientenakten im Stichtagsverfahren als 'angemessen' bewertet ( $R V=95,1 \%)$, davon im Stichtagsverfahren 21,2 \% der Fälle als 'teilweise angemessen' (RV = 27,2 \%). Im Stichtagsverfahren wurden die therapeutischen Maßnahmen in 4,2 \% (RV = 4 \%) als 'nicht angemessen' bewertet. 


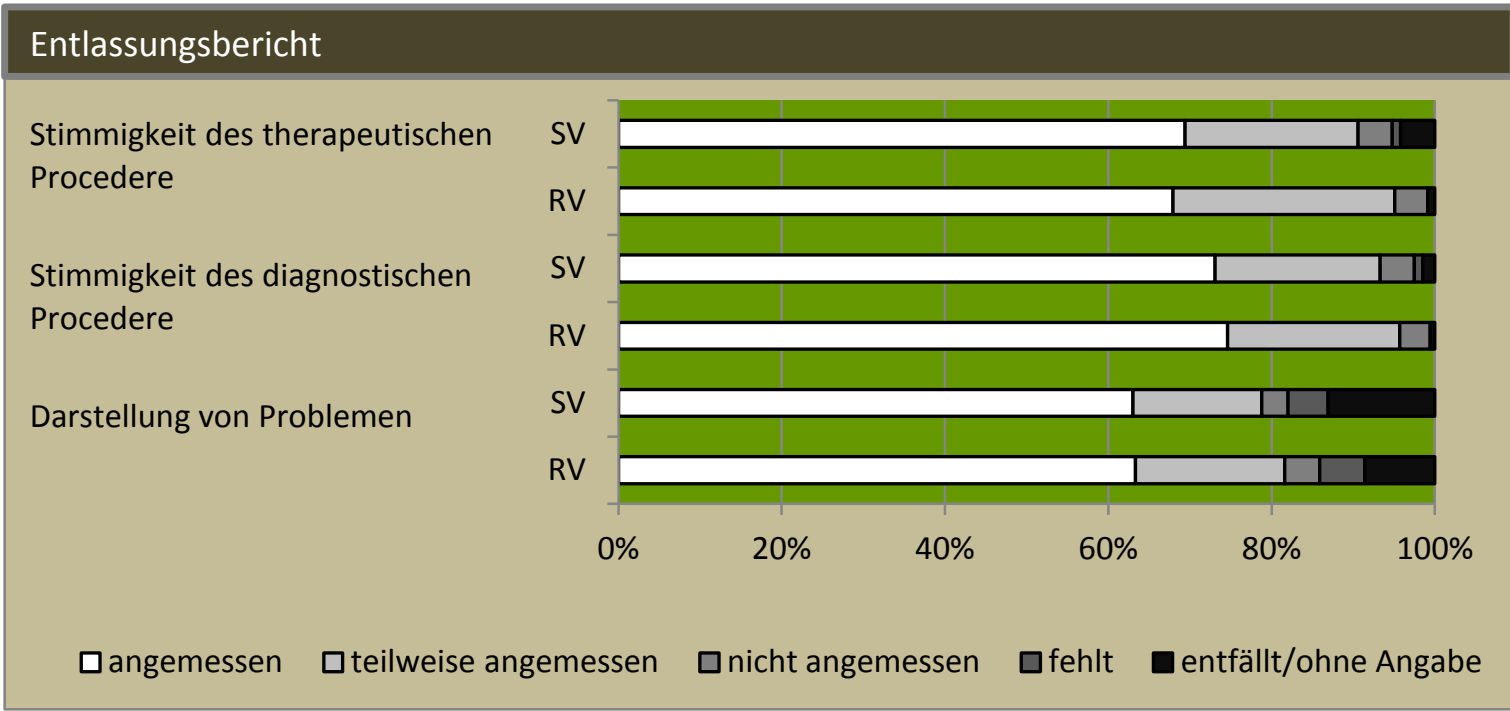

Abbildung 12. Häufigkeitsverteilung der erfassten Items aus dem Beurteilungsfeld Entlassungsbericht für die weiterhin akkreditierten Schlaflaboratorien.

${ }^{*} \mathrm{SV}=$ Stichtagsverfahren, ${ }^{*} \mathrm{RV}=$ Randomisiertes Verfahren

Die Darstellung von aufgetreten „Problemen“ (z. B. Komplikationen, First Night Effekt, Compliance etc.) während der Behandlung 'fehlte' im Stichtagsverfahren in $4,9 \%$ der Fälle (RV $=5,5 \%$ ). Diese war in 3,2 \% der Patientenakten 'nicht angemessen' (RV $=4,3 \%)$.

\subsubsection{Schlaflaboratorien ohne Weiterakkreditierung - nicht verfah- rensbezogen}

An dieser Stelle sollen nur einige Items zur Darstellung kommen. Die vollständigen Zahlen können im Anhang 8.8 entnommen werden.

Im Vergleich zu den Patientenakten der Schlaflaboratorien, die weiterhin akkreditiert bleiben konnten, wurden in Patientenakten der Schlaflaboratorien, die den Akkreditierungsstatus aberkannt bekommen haben $(\mathrm{N}=69)$, die Items aus dem Beurteilungsfeld Anamnese drei bis viermal häufiger vom Gutachter als 'nicht vorhanden' bewertet. Die am häufigsten als 'nicht vorhanden' erfassten Items bezogen sich auf die Angaben der "organischen, psychischen, vegetativen Beschwerden“ und der „Psychosozialen Belastung“. Diese Angaben waren für den Gutachter zwischen $59 \%$ und $81 \%$ der Fälle aus den Patientenakten nicht ersichtlich. Ebenfalls wurde das Item „Risikofaktoren/Suchtanamnese“ besonders häufig als 'nicht vorhanden' codiert. 
Im Beurteilungsfeld Diagnostik wurden besonders häufig die Items „klinisches Labor" (60,9 \%), „Allgemeiner körperlicher, neurologischer, psychiatrischer Status“ $(49,3 \%)$ und „Notwendige Zusatzuntersuchungen“ (44,1\%) vom Gutachter als 'nicht vorhanden' beurteilt. In $34 \%$ der Patientenakten wurden nach Meinung der Gutachter „komorbide Störungen 'nicht erkannt'.

Die Messgrößen („Globale Maße“, „Latenzen“, „Prozentuale Anteile“, „Atmung“, "Lage“, „Beinbewegungen“, „Arousal“) wurden im Schnitt in 82 \% der Patientenakten "teilweise" bis "vollständig" erfasst. Die oben genannten Items 'fehlten' im Schnitt in $18 \%$ der Akten, wobei die meisten fehlenden Angaben die "Lage“ (30\%) und „Beinbewegungen“ (24\%) betrafen. Das „Nachtprotokoll“ wurde in 19 $\%$ der Patientenakten als „nicht verwertbar“ beurteilt.

Aus dem Beurteilungsfeld Therapie wurden die „Allgemeinen Behandlungsmaßnahmen" am häufigsten mit 'fehlend' beurteilt (36,9 \%).

Die "Stimmigkeit des diagnostischen Procedere" wurde in $46 \%$ als 'teilweise angemessen' und in $22 \%$ als 'nicht angemessen' bzw. 'fehlend' vom Gutachter bewertet. Die „Stimmigkeit des therapeutischen Procedere“ erhielt in $15 \%$ die Beurteilung 'nicht angemessen' bzw. 'fehlend'.

\subsubsection{Ergebnis der visuellen Analogskala}

Am Ende der Checkliste beurteilte jeder Gutachter mittels visueller Analogskala, ob der Gesamtprozess der Patientenakte dem aktuellen Stand wissenschaftlicher Erkenntnisse, Empfehlungen und Leitlinien entspricht. Die Werte wurden einer Wertung der Prozessqualität zugeordnet (vgl. 2.8.2 Datenanalyse).

Dabei zeigte sich, dass im Stichtagsverfahren 42,8 \% der Beurteilungen eine "gute" bis „sehr gute" Prozessqualität präsentierten, 39,3\% eine "durchschnittliche" und 17,9 \% eine eher "schwach ausgeprägte bzw. defizitäre" Beurteilung erhielten (Abb.13). Diese Ergebnisse beziehen sich auf die Prozessqualität der beurteilten Patientenakten im Stichtagsverfahren.

Die Frage, in wie vielen Schlafzentren Werte bezüglich des Gesamtprozesses von unter 60 vorlagen, lässt sich folgendermaßen beantworten. In 34 von 149 (22,8 \%) Schlaflaboratorien kam dies mindestens in einer Patientenakte vor. In 14 (9,4 \%) Schlafzentren waren mindestens zwei Patientenakten mit „schwach ausgeprägt 
bzw. defizitär" beurteilter Gesamtqualität. In 6 (4 \%) Schlaflaboratorien beurteilten alle drei Gutachter die Prozessqualität mit „schwach ausgeprägt bzw. defizitär“.

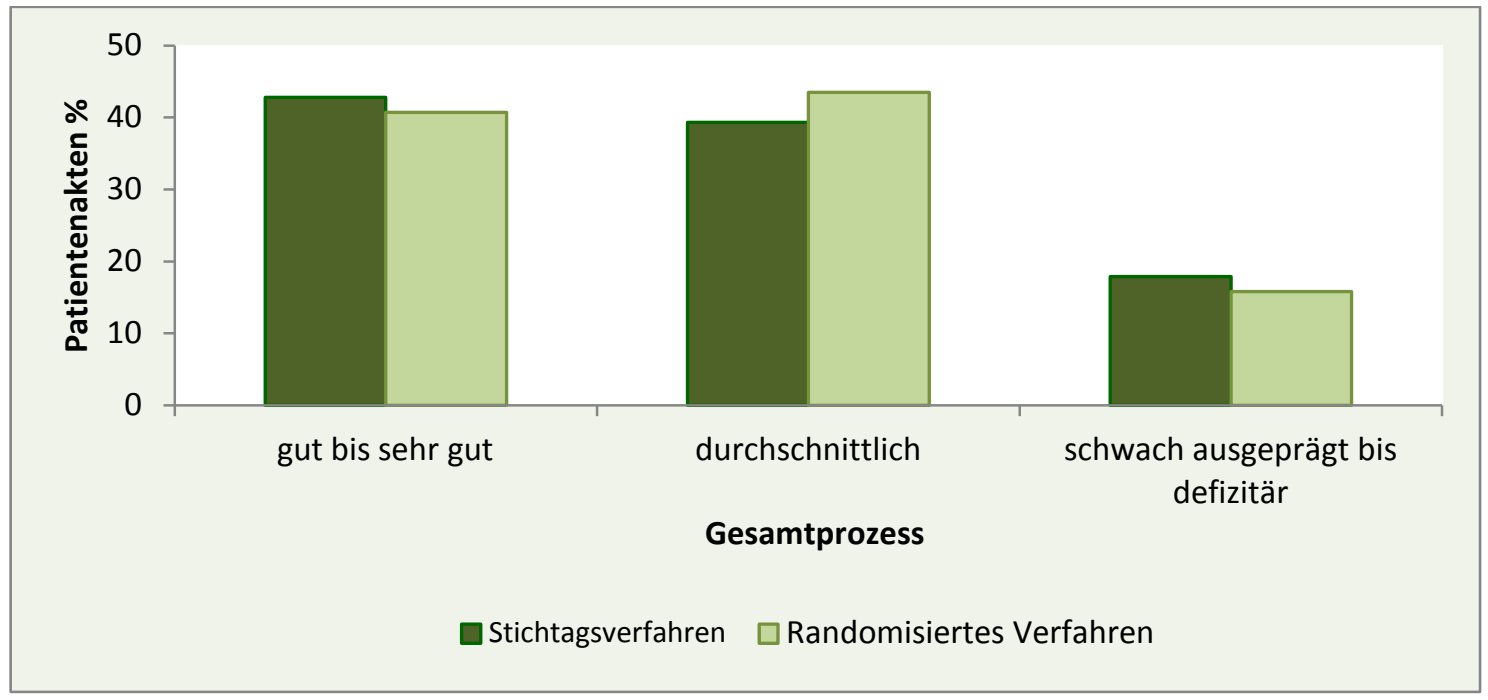

Abbildung 13. Beurteilung des Gesamtprozesses durch die Gutachter mittels visueller Analogskala.

Angelehnt an die Aufteilung Fischer et al. 2006 (S. 488) in die Werte $>80=$ "gute bis sehr gute" Prozessqualität, $60-80=$ "durchschnittliche" Prozessqualität und $<60=$,schwach ausgeprägte bis defizitäre“ Prozessqualität

Im Randomisierten Verfahren zeigte sich ein ähnliches Resultat. 40,7 \% der bewerteten Patientenakten wiesen mit Werten $>80$ eine "gute bis sehr gute" Prozessqualität und somit dem aktuellen Stand wissenschaftlicher Erkenntnisse entsprechend auf.

Der Anteil mit als „durchschnittlich“ beurteilter Gesamtqualität lag bei 43,5\%. In 15,8 \% der Patientenakten wurde die Gesamtqualität eher als „schwach ausgeprägt bzw. defizitär" beurteilt.

Der Gesamtprozess in 3 Patientenakten ( $N=354$ ) des Randomisierten Verfahrens entsprach „überhaupt nicht“ dem aktuellen Stand wissenschaftlicher Erkenntnisse und Leitlinien (Beurteilung"0").

Auf der Ebene der Schlaflaborbeurteilung zeigte sich, dass in $28,8 \%(\mathrm{~N}=118)$ der Schlaflaboratorien mindestens ein Gutachter den Gesamtprozess mit unter 60 bewertete, in 8 Schlaflaboratorien zwei Gutachter. In 2 Schlafzentren waren alle drei Gutachter der Meinung, dass der Gesamtprozess eher eine „schwach ausgeprägte bzw. defizitäre" Leistung vorwies. 
Insgesamt lässt sich anmerken, dass eine schlechte Bewertung im Gesamtprozess nicht mit einer Revisitationsempfehlung korreliert. Es gibt Schlafzentren, die in allen drei Patientenakten Werte unter 60 aufwiesen und dennoch keine einzige Revisitationsempfehlung erhalten haben.

\subsection{Untersuchung der Items hinsichtlich ihrer Einflussnahme auf die Entscheidung des Gutachters zur Revisitation}

Eine zentrale Frage dieser Arbeit sollte sein: Lassen sich Items bestimmen, die den Gutachter zu der Entscheidung bringen, einem Schlaflabor eine Empfehlung zur Revisitation auszusprechen oder ist es das Zusammenspiel vieler Items?

Mit Hilfe des Vierfeldertests als Spezialfall des Chi ${ }^{2}$-Tests wurde untersucht, ob eine Empfehlung zur Revisitation abhängig davon ist, ob Item X 'vorhanden' bzw. 'nicht vorhanden' ist. Jedes einzelne Item wurde zunächst abhängig vom Verfahren untersucht. Die in der Tabelle 10 aufgelisteten p-Werte zeigen für die meisten Items hoch signifikante Ergebnisse. Nur die Items Messgrößenangaben zur „prozentualen Schlafstadienanteile, Atmung, Lage und Arousal“ und das Item „Nachtprotokoll" zeigten keine Abhängigkeit zur Revisitationsentscheidung des Gutachters (siehe Tab. 10). Nach Anwendung des nach Bonferroni-Korrektur angepassten a-Niveaus erscheinen zusätzlich die Items „Risikofaktoren/Suchtanamnese“, „Leistungs-, Vigilanzuntersuchung“ und Messgrößen zu „Globalen Maßen, Latenzen, Beinbewegungen“, sowie „Kontrolluntersuchung“ und die „Verwendung der Standardnomenklatur" als nicht signifikant. 
Tabelle 10.Untersuchung der Abhängigkeit zwischen Itembewertung und der Revisitationsentscheidung des Gutachters mit Hilfe eines $\mathrm{Chi}^{2}$ Vierfeldertests

p- und $X^{2}$-Werte für die einzelnen methodischen Verfahren

\begin{tabular}{|c|c|c|}
\hline Item & $\begin{array}{l}\text { p-Wert }\left(X^{2}-\text { Wert }\right) \\
\text { Stichtagsverfahren }\end{array}$ & $\begin{array}{l}\text { p-Wert ( } \mathrm{X}^{2} \text {-Wert) } \\
\text { Randomisiertes Verfahren }\end{array}$ \\
\hline Allgemeine Anamnese & $5,1^{\mathrm{E}-09}(34,14)$ & $5^{\mathrm{E}-05}(16,54)$ \\
\hline Spezifische Anamnese Nacht & $3,19^{\mathrm{E}-12}(48,57)$ & $2^{\mathrm{E}-08}(31,68)$ \\
\hline Spezifische Anamnese Tag & $8,6^{\mathrm{E}-13}(51,14)$ & $5^{\mathrm{E}-07}(25,46)$ \\
\hline Organische Beschwerden & $1,02^{\mathrm{E}-09}(37,30)$ & $2^{\mathrm{E}-04}(14,26)$ \\
\hline Psychische Beschwerden & $6,9^{\mathrm{E}-08}(29,10)$ & $3^{\mathrm{E}-04}(13,27)$ \\
\hline Vegetative Beschwerden & $1,3^{\mathrm{E}-10}(41,29)$ & $2^{\mathrm{E}-07}(27,31)$ \\
\hline Psychosoziale Belastungen & $1,3^{\mathrm{E}-07}(27,86)$ & $1^{\mathrm{E}-07}(27,86)$ \\
\hline Risikofaktoren/Suchtanamnese & $1,04^{\mathrm{E}-11}(46,25)$ & $0,009(6,76)$ \\
\hline Bisherige Maßnahmen/DD diskutiert & $4,62^{\mathrm{E}-10}(38,83)$ & $1^{\mathrm{E}-05}(19,27)$ \\
\hline Subjektive Befindlichkeit & $2,61^{\mathrm{E}-05}(17,69$ & $0,001(10,78)$ \\
\hline $\begin{array}{l}\text { Allgemeiner körperlicher, neurolo- } \\
\text { gischer, psychiatrischer Status }\end{array}$ & $2,11^{\mathrm{E}-08}(31,39)$ & $2^{\mathrm{E}-04}(13,74)$ \\
\hline Größe, Gewicht, BMI & $7,41^{\mathrm{E}-05}(15,70)$ & $4^{\mathrm{E}-08}(29,93)$ \\
\hline Klinisches Labor & $3,86^{\mathrm{E}-05}(16,94)$ & $1^{\mathrm{E}-05}(18,97)$ \\
\hline Leistungs-, Vigilanzuntersuchung & $0,0003(12,91)$ & $0,004(8,301)$ \\
\hline $\begin{array}{l}\text { Kardioresp. Polysomnographie/ } \\
\text { Polygraphie oder Monitoring }\end{array}$ & $0,072(3,24)$ & $0,001(10,5)$ \\
\hline $\begin{array}{l}\text { Komorbide Störungen erkannt/ di- } \\
\text { agnostiziert }\end{array}$ & $1,18^{\mathrm{E}-10}(41,51)$ & $9^{\mathrm{E}-15}(60)$ \\
\hline Notwendige Zusatzuntersuchung & $1,82^{\mathrm{E}-13}(54,19)$ & $4^{\mathrm{E}-10}(39,17)$ \\
\hline Nachtprotokoll & $0,005(8,04)$ & $0,394(0,727)$ \\
\hline Messgrößen, Globale Maße & $4,85^{\mathrm{E}-08}(29,77)$ & $0,021(5,33)$ \\
\hline Latenzen & $0,0008(11,35)$ & $0,021(5,3)$ \\
\hline Prozentuale Anteile & $0,0008(11,21)$ & $0,619(0,248)$ \\
\hline Atmung, Sättigung, Schnarchen & $0,19(1,72)$ & $0,423(0,643)$ \\
\hline Lage & $0,19(1,72)$ & $0,055(3,688)$ \\
\hline Beinbewegungen & $0,0021(9,43)$ & $0,033(4,56)$ \\
\hline Arousal & $2,23^{\mathrm{E}-05}(17,98)$ & $0,314(1,013)$ \\
\hline $\begin{array}{l}\text { Behandlungsmaßnahmen: Allge- } \\
\text { mein }\end{array}$ & $3,6^{\mathrm{E}-09}(34,83)$ & $2^{\mathrm{E}-04}(14,16)$ \\
\hline
\end{tabular}




\begin{tabular}{|c|c|c|}
\hline Item & $\begin{array}{l}\text { p-Wert ( } X^{2} \text {-Wert) } \\
\text { Stichtagsverfahren }\end{array}$ & $\begin{array}{l}\text { p-Wert ( } \mathbf{X}^{2} \text {-Wert) } \\
\text { Randomisiertes Verfahren }\end{array}$ \\
\hline $\begin{array}{l}\text { Psychologisch/ Psychotherapeu- } \\
\text { tisch }\end{array}$ & $4,94^{\mathrm{E}-05}(16,47)$ & $2^{\mathrm{E}-08}(31,31)$ \\
\hline Medikamentös & $4,76^{\mathrm{E}-05}(16,54)$ & $0,0003(12,55)$ \\
\hline Apparativ 1. Therapienacht & $4,78^{\mathrm{E}-10}(38,76)$ & $4,78^{\mathrm{E}-10}(38,76)$ \\
\hline Kontrolluntersuchung & $0,003(8,64)$ & $5,46^{\mathrm{E}-06}(20,67)$ \\
\hline $\begin{array}{l}\text { Verwendung der Standardnomen- } \\
\text { klatur }\end{array}$ & $0,009(6,83)$ & $6,13^{\mathrm{E}-05}(16,06)$ \\
\hline $\begin{array}{l}\text { Stimmigkeit des diagnostischen } \\
\text { Procedere }\end{array}$ & $5,58^{\mathrm{E}-16}(65,58)$ & $3,48^{\mathrm{E}-22}(93,80)$ \\
\hline $\begin{array}{l}\text { Zusammenfassende Beurteilung } \\
\text { Schlafparameter }\end{array}$ & $6,22^{\mathrm{E}-15}(60,83)$ & $6,13^{\mathrm{E}-05}(16,06)$ \\
\hline Atmung, $\mathrm{SaO} 2, \ldots$ & $3,2^{\mathrm{E}-12}(48,56)$ & $1,06^{\mathrm{E}-10}(41,71)$ \\
\hline Beinbewegung & $2,32^{\mathrm{E}-10}(40,17)$ & $2,6^{\mathrm{E}-09}(35,47)$ \\
\hline $\begin{array}{l}\text { Stimmigkeit des therapeutischen } \\
\text { Procedere }\end{array}$ & $4,2^{\mathrm{E}-13}(52,54)$ & $9,22^{\mathrm{E}-11}(41,98)$ \\
\hline Darstellung von Problemen & $6,44^{\mathrm{E}-15}(60,76)$ & $3,28^{\mathrm{E}-11}(44,00)$ \\
\hline
\end{tabular}

Im nächsten Schritt wurde nach dem mathematischen Zusammenhang zwischen einem 'nicht vorhandenen' Item und einer Revisitationsempfehlung gesucht. Lässt sich also eine Korrelation diesbezüglich nachweisen?

Dazu werden im Folgenden die Häufigkeitsverteilungen in Tabelle 11 bis 15 angegeben. In Klammern ist jeweils die Anzahl der für die Berechnung zur Verfügung stehenden Patientenakten dargelegt. Wie in Kapitel 4.2 dargestellt, ließ sich kein Unterschied bezüglich des Akkreditierungsergebnisses zwischen den beiden angewandten Verfahren feststellen. Aus diesem Grund wurden die Daten beider Verfahren für die weiteren Analysen gemeinsam betrachtet und zusammengefasst beurteilt. In der Betrachtung wird sich vor allem auf die Untersuchung der negativen Beurteilung ('nicht vorhanden', 'Empfehlung fehlt', 'nicht angemessen') konzentriert. Der Vollständigkeitshalber sind aber die gesamten Werte in den Tabellen aufgeführt.

Bei dem Beurteilungsfeld Anamnese fällt auf, dass Patientenakten mit fehlenden Angaben zur "Spezifischen Anamnese" besonders häufig eine Revisitationsempfehlung erhielten (siehe Tab. 11). Die Hälfte der Akten (51,5\% Tag und 46,3 \% Nacht), in denen die „Spezifische Anamnese“ bei „Tag“ und „Nacht“ 'nicht 
vorhanden' waren, erhielten eine Revisitationsempfehlung. Die geringste Anzahl der Revisitationsempfehlungen trotz des fehlenden Items aus dem Beurteilungsfeld Anamnese konnte bei dem Prozessmerkmal „Psychische Beschwerden“ (24,5 \%) bzw. „Psychosoziale Belastungen“ (24,8 \%) beobachtet werden.

Von den Patientenakten, in denen die „Allgemeine Anamnese“ 'nicht vorhanden' war, haben $65 \%$ der Akten keine Empfehlung zur Revisitation erhalten. Ferner ließ sich feststellen, dass 6-10\% der Patientenakten eine Revisitationsempfehlung erhielten, obwohl jeweils das Item aus dem Beurteilungsfeld Anamnese vorzufinden war. Umgekehrt fällt auf, dass im Schnitt 65,5 \% der Patientenakten keine Revisitationsempfehlung erhielten, obwohlt Items aus dem Beurteilungsfeld Anamnese fehlten.

Tabelle 11.Überblick über die beurteilten Prozessmerkmale (Anamnese) und ihre Häufigkeitsverteilung im Hinblick auf die Revisitationsentscheidung des Gutachters.

Ausgehend von der Beurteilung des Items wird die Gesamtheit der Patientenakten mit bzw. ohne Revisitationsempfehlung mit $100 \%$ angegeben.

\begin{tabular}{|c|c|c|c|}
\hline Anamnese & & $\begin{array}{c}\text { Patientenakten ohne } \\
\text { Revisitationsempfehlung (N) }\end{array}$ & $\begin{array}{c}\text { Patientenakten mit } \\
\text { Revisitationsempfehlung (N) }\end{array}$ \\
\hline \multirow{2}{*}{ Allgemeine Anamnese } & nicht vorhanden & $64,8 \%(68)$ & $35,2 \%(37)$ \\
\hline & vorhanden*1 & $89,8 \%(615)$ & $10,2 \%(70)$ \\
\hline \multirow{2}{*}{$\begin{array}{c}\text { Spezifische Anamnese } \\
\text { Nacht }\end{array}$} & nicht vorhanden & $53,7 \%(44)$ & $46,3 \%(38)$ \\
\hline & vorhanden*1 & $90,5 \%(639)$ & $9,5 \%(67)$ \\
\hline \multirow{2}{*}{ Spezifische Anamnese Tag } & nicht vorhanden & $48,5 \%(32)$ & $51,5 \%(34)$ \\
\hline & vorhanden*1 & $89,9 \%(652)$ & $10,1 \%(73)$ \\
\hline \multirow{2}{*}{ Org. Beschwerden } & nicht vorhanden & $68,1 \%(96)$ & $31,9 \%(45)$ \\
\hline & vorhanden*1 & $91 \%(584)$ & $9 \%(58)$ \\
\hline \multirow{2}{*}{ Psych. Beschwerden } & nicht vorhanden & $75,5 \%(200)$ & $24,5 \%(65)$ \\
\hline & vorhanden*1 & $92,2 \%(462)$ & $7,8 \%(39)$ \\
\hline \multirow{2}{*}{ Veg. Beschwerden } & nicht vorhanden & $71,6 \%(169)$ & $28,4 \%(67)$ \\
\hline & vorhanden*1 & $93,2 \%(504)$ & $6,8 \%(37)$ \\
\hline \multirow{2}{*}{ Psychosoz. Belastungen } & nicht vorhanden & $75,2 \%(227)$ & $24,8 \%(75)$ \\
\hline & vorhanden*1 & $94 \%(435)$ & $6 \%(28)$ \\
\hline \multirow{2}{*}{$\begin{array}{l}\text { Risikofaktoren } \\
\text { /Suchtanamnese }\end{array}$} & nicht vorhanden & $73,4 \%(168)$ & $26,6 \%(61)$ \\
\hline & vorhanden¹ & $92,1 \%(493)$ & $7,9 \%(42)$ \\
\hline \multirow{2}{*}{$\begin{array}{c}\text { Bisherige Maßnahmen } \\
\text { diskutiert }\end{array}$} & nicht vorhanden & $69,3 \%(115)$ & $30,7 \%(51)$ \\
\hline & vorhanden*1 & $91,5 \%(546)$ & $8,5 \%(51)$ \\
\hline
\end{tabular}

Die als 'nicht vorhanden' beurteilten Items aus dem Beurteilungsfeld Diagnostik waren nur etwa bei einem Drittel der Patientenakten tatsächlich mit einer Revisitationsempfehlung assoziiert (vgl. Tab. 12). Von den geprüften Patientenak- 
ten der Schlaflaboratorien, bei denen der Vermerk zur "Subjektiven Befindlichkeit“ als 'fehlend' beurteilt wurde, erhielten $70 \%$ trotzdem keine Revisitationsempfehlung. Ebenso fält besonders auf, dass $76 \%$ der Patientenakten trotz fehlenden Angaben zum „klinischen Labor" keine Revisitationsempfehlung erhielten.

Von den Akten, in denen die Angaben zu Körpermaßen („Gewicht, Größe, BMI“) 'fehlten', erhielten $66 \%$ keine Revisitationsempfehlung.

In den meisten Fällen wurden Revisitationsempfehlungen ausgesprochen, wenn in den Patientenakten „Komorbide Störungen“ „nicht erkannt“ bzw. „diagnostiziert" wurden. $43 \%$ der Patientenakten, in denen dies nicht geschah, erhielten eine Empfehlung zur Revisitation.

Tabelle 12.Gegenüberstellung der erfassten Prozessmerkmale (Diagnostik) und ihre Häufigkeitsverteilung im Hinblick auf die Revisitationsentscheidung des Gutachters.

Ausgehend von der Beurteilung des Items wird die Gesamtheit der Patientenakten mit bzw. ohne Revisitationsempfehlung mit $100 \%$ angegeben.

\begin{tabular}{|c|c|c|c|}
\hline Diagnostik & & $\begin{array}{c}\text { Patientenakten ohne } \\
\text { Revisitationsempfehlung (N) }\end{array}$ & $\begin{array}{c}\text { Patientenakten mit } \\
\text { Revisitationsempfehlung (N) }\end{array}$ \\
\hline \multirow{2}{*}{ Subjektive Befindlichkeit } & nicht vorhanden ${ }^{* 1}$ & $69,6 \%(78)$ & $30,4 \%(34)$ \\
\hline & vorhanden*2 & $89,8 \%(581)$ & $10,2 \%(66)$ \\
\hline \multirow{2}{*}{$\begin{array}{l}\text { Allgemeiner, neurolog, } \\
\text { psychiatrischer Status }\end{array}$} & nicht vorhanden ${ }^{\star 1}$ & $67,4 \%(91)$ & $32,6 \%(44)$ \\
\hline & vorhanden*2 & $90,8 \%(593)$ & $9,2 \%(60)$ \\
\hline \multirow{2}{*}{ Größe, Gewicht, BMI } & nicht vorhanden ${ }^{* 1}$ & $65,7 \%(71)$ & $34,3 \%(37)$ \\
\hline & vorhanden ${ }^{* 2}$ & $90 \%(610)$ & $10 \%(68)$ \\
\hline \multirow{2}{*}{ Klinisches Labor } & nicht vorhanden*1 & $75,9 \%(189)$ & $24,1 \%(60)$ \\
\hline & vorhanden*2 & $91,3 \%(417)$ & $8,8 \%(40)$ \\
\hline \multirow{2}{*}{$\begin{array}{l}\text { Leistungs-, } \\
\text { Vigilanzuntersuchung }\end{array}$} & nicht vorhanden*1 & $80 \%(260)$ & $20 \%(65)$ \\
\hline & vorhanden ${ }^{* 2}$ & $92,5 \%(248)$ & $7,5 \%(20)$ \\
\hline \multirow{2}{*}{$\begin{array}{c}\text { Kardioresp. } \\
\text { Polysomnographie/ } \\
\text { Polygraphie oder Monitoring }\end{array}$} & nicht vorhanden ${ }^{\star 1}$ & $72,8 \%(59)$ & $27,2 \%(22)$ \\
\hline & vorhanden*2 & $88,1 \%(597)$ & $12 \%(81)$ \\
\hline \multirow{2}{*}{$\begin{array}{l}\text { Komorbide Störungen } \\
\text { erkannt/diagnostiziert }\end{array}$} & nicht vorhanden ${ }^{* 1}$ & $57 \%(57)$ & $43 \%(43)$ \\
\hline & vorhanden ${ }^{\star 2}$ & $91,5 \%(541)$ & $8,5 \%(50)$ \\
\hline \multirow{2}{*}{$\begin{array}{l}\text { Notwendige } \\
\text { Zusatzuntersuchung }\end{array}$} & nicht vorhanden*1 & $63,3 \%(95)$ & $36,7 \%(55)$ \\
\hline & vorhanden*2 & $91,4 \%(363)$ & $8,6 \%(34)$ \\
\hline
\end{tabular}

${ }^{* 1}$ nicht vorhanden, Empfehlung fehlt

${ }^{* 2}$ vorhanden, bereits extern durchgeführt, Empfehlung ist erfolgt 
Ein hoher Prozentsatz der Patientenakten, in denen die Dokumentation der Messgrößen 'fehlte', haben dennoch keine Revisitationsempfehlung erhalten (siehe Tab. 13).

Tabelle 13.Vergleich der bewerteten Prozessmerkmale (Diagnosesicherung) und ihre Häufigkeitsverteilung im Hinblick auf die Revisitationsentscheidung des Gutachters.

Ausgehend von der Beurteilung des Items wird die Gesamtheit der Patientenakten mit bzw. ohne Revisitationsempfehlung mit $100 \%$ angegeben.

\begin{tabular}{|c|c|c|c|}
\hline Diagnosesicherung & & $\begin{array}{c}\text { Patientenakten ohne } \\
\text { Revisitationsempfehlung (N) }\end{array}$ & $\begin{array}{c}\text { Patientenakten mit } \\
\text { Revisitationsempfehlung (N) }\end{array}$ \\
\hline \multirow{2}{*}{ Nachtprotokoll } & nicht verwertbar & $76,4 \%(42)$ & $23,6 \%(13)$ \\
\hline & vollständig*1 & $88,1 \%(620)$ & $11,9 \%(84)$ \\
\hline \multirow{2}{*}{$\begin{array}{l}\text { Messgrößen } \\
\text { Globale Maße }\end{array}$} & fehlt & $50 \%(12)$ & $50 \%(12)$ \\
\hline & vollständig*1 & $88,3 \%(672)$ & $11,7 \%(89)$ \\
\hline \multirow{2}{*}{ Latenzen } & fehlt & $69,6 \%(39)$ & $30,3 \%(17)$ \\
\hline & vollständig*1 & $88,3 \%$ (639) & $11,7 \%(85)$ \\
\hline \multirow{2}{*}{ Prozentuale Anteile } & fehlt & $70,3 \%(26)$ & $29,7 \%(11)$ \\
\hline & vollständig*1 & $87,9 \%(659)$ & $12,1 \%(91)$ \\
\hline \multirow{2}{*}{$\begin{array}{l}\text { Atmung, Sättigung, } \\
\text { Schnarchen }\end{array}$} & fehlt & $60 \%(3)$ & $40 \%(2)$ \\
\hline & vollständig*1 & $87,2 \%(683)$ & $12,8 \%(100)$ \\
\hline \multirow{2}{*}{ Lage } & fehlt & $81,1 \%(120)$ & $18,9 \%(28)$ \\
\hline & vollständig*1 & $88,3 \%(549)$ & $11,7 \%(73)$ \\
\hline \multirow{2}{*}{ Beinbewegung } & fehlt & $73,3 \%(44)$ & $26,7 \%(16)$ \\
\hline & vollständig*1 & $88,1 \%(624)$ & $11,9 \%(84)$ \\
\hline \multirow{2}{*}{ Arousal } & fehlt & $71,7 \%(43)$ & $28,3 \%(17)$ \\
\hline & vollständig*1 & $88,3 \%(633)$ & $11,7 \%(84)$ \\
\hline
\end{tabular}

Im Beurteilungsfeld Therapie muss zunächst darauf verwiesen werden, dass in Patientenakten mit Revisitationsempfehlungen nur ein niedriger Anteil der Items tatsächlich 'fehlte' ( $\mathrm{N}=10-32)$ (siehe Tab. 14). Dadurch ist die Interpretation erschwert.

Es lässt sich kein Item bestimmen, das mit der Entscheidung eines Gutachters zur Revisitation in Zusammenhang gebracht werden könnte. Zwar führte eine 'fehlende' „apparative Therapienacht“ vermehrt zu einer Revisitationsempfehlung (59 \%). 
Aufgrund der niedrigen Fallzahl $(\mathrm{N}=17)$ bezüglich der fehlenden Angaben für dieses Item ist diese Aussage jedoch nicht eindeutig zu treffen.

Tabelle 14.Vergleich der bewerteten Prozessmerkmale (Therapie) und ihre Häufigkeitsverteilung im Hinblick auf die Revisitationsentscheidung des Gutachters.

Ausgehend von der Beurteilung des Items wird die Gesamtheit der Patientenakten mit bzw. ohne Revisitationsempfehlung mit $100 \%$ angegeben.

\begin{tabular}{|c|c|c|c|}
\hline \multicolumn{2}{|l|}{ Therapie } & $\begin{array}{c}\text { Patientenakten ohne } \\
\text { Revisitationsempfehlung (N) }\end{array}$ & $\begin{array}{c}\text { Patientenakten mit } \\
\text { Revisitationsempfehlung (N) }\end{array}$ \\
\hline \multirow{2}{*}{$\begin{array}{l}\text { Behandlungsmaßnahmen- } \\
\text { Allgemein }\end{array}$} & fehlt & $65,2 \%(60)$ & $34,8 \%$ (32) \\
\hline & durchgeführt $^{\star 1}$ & $91 \%(574)$ & $9 \%(57)$ \\
\hline \multirow{2}{*}{$\begin{array}{c}\text { Psychologisch, } \\
\text { psychotherapeutisch }\end{array}$} & fehlt & $66,7 \%(70)$ & $33,3 \%(35)$ \\
\hline & durchgeführt $^{\star 1}$ & $93,3 \%(168)$ & $6,7 \%(12)$ \\
\hline \multirow{2}{*}{ Medikamentös } & fehlt & $63,8 \%(37)$ & $36,2 \%(21)$ \\
\hline & durchgeführt $^{\star 1}$ & $93,2 \%(192)$ & $6,8 \%(14)$ \\
\hline \multirow{2}{*}{ Apparativ $^{* 2}$ 1. Therapienacht } & fehlt & $41,2 \%(7)$ & $58,8 \%(10)$ \\
\hline & durchgeführt ${ }^{\star 1}$ & $87,8 \%(447)$ & $12,2 \%(62)$ \\
\hline \multirow{2}{*}{ Kontrolluntersuchung } & fehlt & $65,6 \%(42)$ & $34,4 \%(22)$ \\
\hline & durchgeführt¹ & $88,2 \%(469)$ & $11,8 \%(63)$ \\
\hline
\end{tabular}

${ }^{* 1}$ durchgeführt, terminiert, empfohlen

${ }^{* 2}$ die 2. Therapienachtkommtnichtzur Darstellung, weil diese nur in sehr selten Fällen dokumentiertwurde

Im Beurteilungsfeld Entlassungsbericht zeigt sich ein auffälliger Zusammenhang zwischen den als 'nicht angemessen' beurteilten Items "Stimmigkeit des diagnostischen Procedere" und "Stimmigkeit des therapeutischen Procedere" und einer Revisitationsentscheidung (vgl. Tab. 15).

$67 \%$ der Patientenakten, in denen das Item „Stimmigkeit des diagnostischen Procedere" als 'fehlend' bzw. 'nicht angemessen' beurteilt wurde, erhielten eine Revisitationsempfehlung. In 60,5\% der Patientenakten, in denen das Item „Stimmigkeit des therapeutischen Procedere" als 'fehlend' bzw. 'nicht angemessen' erfasst wurde, wurde eine Revisitationsempfehlung ausgesprochen (siehe Tab. 15). Interessant ist auch der Vergleich der absoluten Häufigkeit von Patientenakten ohne Revisitationsempfehlung ( $N=16$ von $685=>2 \%$ ) mit den Patientenakten mit 
Revisitationsempfehlung ( $\mathrm{N}=32$ von $105=>30 \%$ ) bei denen das Item „Stimmigkeit des diagnostischen Procedere" als 'nicht angemessen' beurteilt wurde (siehe Tab. 15). Im Ergebnis wurde bei Patientenakten mit Revisitationsempfehlung 15mal häufiger (30 \% zu 2 \%) das Item „Stimmigkeit des diagnostischen Procedere“ als 'nicht angemessen' beurteilt.

Das Item "Stimmigkeit des therapeutischen Procedere" wurde 8-mal häufiger in den Patientenakten mit Revisitationsempfehlung ( $N=26$ von $104=>25 \%)$ als 'nicht angemessen' beurteilt im Vergleich zu Patientenakten ohne Empfehlung zur Revisitation ( $N=17$ von $672=>3 \%$ ) (vgl. Tab. 15).

$46,7 \%$ der Patientenakten, in denen laut Gutachter die „Darstellung von Problemen“ 'nicht angemessen' erschien, wurden mit einer Revisitationsempfehlung versehen.

$51,3 \%$ der Patientenakten, in denen die „Zusammenfassende Beurteilung der Schlafparameter" als 'nicht angemessen' bewertet wurde und $71,4 \%$ der Patientenakten, in denen die „Atmungsparameter“ als 'nicht angemessen' dokumentiert wurden, waren ebenfalls mit einer Revisitationsempfehlung verbunden.

\section{Tabelle 15.Gegenüberstellung der bewerteten Prozessmerkmale (Entlassungsbericht) und ihre Häufigkeitsverteilung im Hinblick auf die Revisitationsentscheidung des Gutachters.}

Ausgehend von der Beurteilung des Items wird die Gesamtheit der Patientenakten mit bzw. ohne Revisitationsempfehlung mit $100 \%$ angegeben.

\begin{tabular}{|c|c|c|c|}
\hline \multicolumn{2}{|l|}{ Entlassungsbericht } & $\begin{array}{l}\text { Patientenakten ohne } \\
\text { Revisitationsempfehlung (N) }\end{array}$ & $\begin{array}{c}\text { Patientenakten mit } \\
\text { Revisitationsempfehlung (N) }\end{array}$ \\
\hline \multirow{2}{*}{$\begin{array}{c}\text { Verwendung der } \\
\text { Standardnomenklatur }\end{array}$} & nicht angemessen ${ }^{* 1}$ & $46,9 \%(15)$ & $53,1 \%(17)$ \\
\hline & angemessen ${ }^{\star 2}$ & $88,3 \%(672)$ & $11,7 \%(89)$ \\
\hline \multirow{2}{*}{$\begin{array}{c}\text { Stimmigkeit des diagnostischen } \\
\text { Procedere }\end{array}$} & nicht angemessen ${ }^{* 1}$ & $33,3 \%(16)$ & $66,7 \%(32)$ \\
\hline & angemessen ${ }^{\star 2}$ & $90,2 \%(669)$ & $9,9 \%(73)$ \\
\hline \multirow{2}{*}{$\begin{array}{l}\text { Zusammenfassende Beurteilung der } \\
\text { a) Schlafparameter }\end{array}$} & nicht angemessen ${ }^{* 1}$ & $48,7 \%(38)$ & $51,3 \%(40)$ \\
\hline & angemessen ${ }^{* 2}$ & $91,1 \%(636)$ & $8,9 \%(62)$ \\
\hline \multirow{2}{*}{ b) Atmung, SaO2,... } & nicht angemessen ${ }^{* 1}$ & $28,6 \%(8)$ & $71,4 \%(20)$ \\
\hline & angemessen ${ }^{\star 2}$ & $88,8 \%(676)$ & $11,2 \%(85)$ \\
\hline \multirow{2}{*}{ c) Beinbewegung } & nicht angemessen ${ }^{* 1}$ & $62,7 \%(79)$ & $37,3 \%(47)$ \\
\hline & angemessen ${ }^{\star 2}$ & $91,2 \%(549)$ & $8,8 \%(53)$ \\
\hline \multirow{2}{*}{$\begin{array}{c}\text { Stimmigkeit des therapeutischen } \\
\text { Procedere }\end{array}$} & nicht angemessen ${ }^{* 1}$ & $39,5 \%(17)$ & $60,5 \%(26)$ \\
\hline & angemessen ${ }^{\star 2}$ & $89,4 \%(655)$ & $10,6 \%(78)$ \\
\hline \multirow{2}{*}{ Darstellung von Problemen } & nicht angemessen ${ }^{* 1}$ & $53,3 \%(48)$ & $46,7 \%(42)$ \\
\hline & angemessen ${ }^{\star 2}$ & $91,5 \%(568)$ & $8,5 \%(53)$ \\
\hline
\end{tabular}


Bei der Frage, ob einzelne Items bestimmt werden können, bei deren Fehlen sicherlich eine Revisitationsempfehlung folgt, ergibt sich folgendes Resultat.

Es können keine einzelnen Items bestimmt werden, bei deren Fehlen es zwangsläufig zur Revisitationsempfehlung kommt. Jedoch lassen sich einige Items bestimmen, bei deren Fehlen vermehrt eine Revisitationsempfehlung folgte.

Gleichzeitig lassen sich Items darstellen, die zu hohem prozentualem Anteil fehlten, ohne eine Revisitationsempfehlung nach sich zu ziehen.

Einzelne fehlende Items führten besonders oft zur Empfehlung einer Revisitation. So scheint aus dem Beurteilungsfeld der Anamnese die "Spezifische Anamnese“ bei Tag und Nacht eine bedeutendere Rolle zu spielen. In Akten mit fehlenden Angaben dazu, erhalten die Hälfte der Akten eine Revisitationsempfehlung.

Aus dem Beurteilungsfeld der Diagnostik sticht zwar kein Item besonders hervor. Es fällt aber auf, dass $43 \%$ der Patientenakten, in denen "Komorbide Störungen“ „nicht erkannt" wurden, eine Revisitationsempfehlung erhalten haben.

Aus dem Beurteilungsfeld der Diagnosesicherung und der Therapie lassen sich keine bedeutungsvollen Items bestimmen.

Einflussreiche Items sind vermehrt im Beurteilungsfeld Entlassungsbericht zu finden. Ein großer Teil der Patientenakten mit 'nicht angemessener' Beurteilung von "Schlaf-, und Atmungsparametern" erhalten eine Revisitationsempfehlung.

Noch deutlicher scheint die unangemessene Beurteilung der diagnostischen und therapeutischen Stimmigkeit hinsichtlich einer Revisitationsempfehlung zu sein. Dabei erhalten die Patientenakten, in denen das diagnostische oder therapeutische Procedere als unangemessen beurteilt wurde, die meisten Revisitationsempfehlungen. 


\section{Diskussion}

\subsection{Evaluierung der methodischen Verfahren}

Das Qualitätssicherungsprogramm 2009/10 der DGSM als Bestandteil der Weiterakkreditierung der Schlaflaboratorien wurde nach zwei unterschiedlichen methodischen Verfahren in Bezug auf die Auswahl der teilnehmenden Patientenakten durchgeführt. In dieser Arbeit konnte gezeigt werden, dass das Stichtagsverfahren mehr den Ansprüchen eines unkomplizierten und mit weniger zusätzlichem Aufwand verbundenen Qualitätssicherungsverfahrens entspricht.

\subsubsection{Ergebnisse Organisationstruktur}

Das Stichtagsverfahren überzeugte durch eine überschaubare Umsetzung der Methodik und demzufolge eine deutliche Zeiteinsparung im Ablauf des Qualitätssicherungsprogramms. Nach Mitteilung der DGSM stellten sich die Schlaflaboratorien ggf. auf Erfordernisse und Dokumentation für vier Wochen ein. Nach Bekanntgabe des Stichtages im Folgemonat konnten die Patientenunterlagen direkt an die Zentralstelle der DGSM zugestellt und zeitnah an die Gutachter verteilt werden. Das Stichtagsverfahren konnte im Großen und Ganzen innerhalb von acht Monaten abgeschlossen werden.

Im Randomisierten Verfahren waren einige Zwischenschritte nötig, die einen reibungslosen Ablauf verzögerten. Die Schlaflaboratorien waren aufgefordert zunächst die aufgestellten Patientenlisten an die Koordinationsstelle der DGSM zu übersenden. Die Verantwortlichen suchten drei Patienten aus und forderten die dazugehörigen Patientenakten an. Schließlich, nach Eingang der Akten in der Zentralstelle, konnten diese an die Gutachter verteilt werden. Der größere organisatorische Aufwand führte zur unnötigen zeitlichen Verzögerung der Begutachtung. Insgesamt war das Qualitätssicherungsverfahren erst nach 16 Monaten abgeschlossen. Demzufolge musste für die Durchführung des Randomisierten Verfahrens der doppelte Zeitaufwand aufgebracht werden.

Durch den aufwendigen Ablauf des Randomisierten Verfahren kommt es zu langen Zeitabständen zwischen Datenerfassung und Ergebnisrückmeldung. Diese Tatsache erschwert eine zeitnahe Reaktion auf bestehende und durch das Programm dokumentierte Qualitätsprobleme. Des Weiteren kann eine aktuelle ge- 
genwärtige Qualität nicht direkt abgebildet werden, da in der Zwischenzeit Veränderungen eingetreten sein können. Definiert man die Zielsetzung des Qualitätssicherungsprogramms zur Verbesserung der Prozessqualität (DGSM 2011), so muss angemerkt werden, dass durch die zeitliche Verzögerung im Randomisierten Verfahren die Wirksamkeit der Mängelbehebung anzuzweifeln ist.

Ein wichtiger Aspekt bezüglich der Dauer des Qualitätssicherungsprogramms spricht zusätzlich gegen das Randomisierte Verfahren. Die Reevaluation der Schlaflaboratorien zur Fortführung des Akkreditierungsstatus wird alle zwei Jahre durchgeführt. Dauert jedoch die Durchführung eines prüfenden Durchganges allein 16 Monate, so liegen nur 8 Monate zwischen den einzelnen Überprüfungen. Auf der einen Seite findet zwar somit eine regelmäßige Überprüfung der Leistungen im Schlaflabor statt. Andererseits erscheint der Zeitraum zur Mängelbehebung recht kurz. Zum anderen wäre es denkbar, dass die Schlaflaboratorien aufgrund der ausgedehnten Verfahrensweise nicht mehr am Qualitätssicherungsprogramm teilnehmen.

Es wäre ebenso denkbar, dass das Bewusstsein für die Mängelbehebung, wenn die Erhebung sehr lange zurück liegt, nicht mehr vorhanden ist.

\subsubsection{Ergebnisse Weiterakkreditierung und Revisitationsentschei- dung}

Es konnte kein signifikanter Unterschied zwischen den beiden Methoden bezüglich des Einflusses auf den Akkreditierungsstatus festgestellt werden. Im Vergleich der Anzahl weiter akkreditiert bleibender Schlafzentren zeigte sich ein identisches Ergebnis. Während im Stichtagsverfahren $9 \%$ der teilnehmenden Schlaflaboratorien den Akkreditierungsstatus der DGSM verlieren, sind es im Randomisierten Verfahren 8 \%. Auch die Häufigkeit der Anzahl der Revisitationsempfehlungen pro schlafmedizinisches Zentrum offenbarte ein annähernd gleiches Ergebnis unabhängig vom durchgeführten Verfahren.

Ob die Patientenakten prospektiv (Stichtagsverfahren) oder retrospektiv (Randomisiertes Verfahren) erhoben wurden, schien keinen Einfluss auf die Revisitationsempfehlung zu nehmen.

Dieses Ergebnis war sehr nahe liegend, da trotz Unterschieden in der Patientenauswahl der beiden Verfahren für die schlafmedizinischen Zentren gleiche Anfor- 
derungen und Bedingungen herrschten. Zum einen war beiden Verfahren die zufällige Patientenauswahl gemeinsam, sodass in keinem Verfahren die drei schließlich teilnehmenden Patientenakten vorher bekannt gewesen sein konnten. Zum anderen besaßen die Schlaflaboratorien in beiden Methoden gleiche Bedingungen hinsichtlich der Dokumentation. Sowohl im Stichtagsverfahren als auch im Randomisierten Verfahren konnte sich auf die definierte Dokumentation eingestellt werden. Insgesamt war für beide Verfahren ein Zeitraum angegeben, sodass die Dokumentation im Endeffekt prospektiv durchgeführt werden musste.

\subsubsection{Ergebnisse der erfassten Items}

Der Anteil der Items, die als 'vorhanden' erfasst wurden, waren im Randomisierten Verfahren minimal höher im Vergleich zum Stichtagsverfahren. Im Randomisierten Verfahren wurden die meisten Items vom Gutachter zwischen 2 und $10 \%$ häufiger als 'vorhanden' erfasst. Die Prozessmerkmale an denen die auffälligste Abweichung zu erkennen war, war u. a. die „Allgemeine Anamnese“ (10 \% häufiger), Angaben zu „Psychischen Beschwerden“ (8 \% häufiger), die „Subjektive Befindlichkeit“ (7 \% häufiger) und das Erkennen „komorbider Störungen“ (7 \% häufiger).

Obwohl mehr Items als 'vorhanden' erfasst werden konnten, schien es rechnerisch keinen großen Einfluss auf die Bewertung der „Stimmigkeit des diagnostischen und therapeutischen Procedere“ zu nehmen. Der Anteil 'angemessener' bzw. 'teilweise angemessener' Beurteilung verändert sich nicht.

Somit ist nicht davon auszugehen, dass im Randomisierten Verfahren Items besser erfasst werden können. Vielmehr liegt die Vermutung nahe, dass tatsächlich in diesem Verfahren ausführlicher dokumentiert wurde. Dies wiederum könnte mit der Veröffentlichung der Daten aus dem ersten Durchgang (Stichtagsverfahren) zusammenhängen. Zum einen erschienen die Daten auf dem „Congress of the European Sleep Research Society 2010“ und zum anderen fand eine Jahresversammlung der DGSM (Oktober 2009) statt. Dort wurden die ersten Ergebnisse des Stichtagsverfahrens vorgestellt mit der Aufforderung, in den Entlassungsbriefen zukünftig gründlicher zu dokumentieren.

Dies könnte ein Grund für die, prozentual gesehen, bessere Quantität der dokumentierten Items im Randomisierten Verfahren darstellen. Ein anderer Zusammenhang scheint nicht offensichtlich. 


\subsubsection{Empfehlung eines Verfahrens zur weiteren Durchführung im Rahmen des Qualitätssicherungsprogramms der DGSM}

Nach Abwägen der Vor- und Nachteile entspricht das Stichtagsverfahren mehr den Ansprüchen eines unkomplizierten und mit weniger zusätzlichem Aufwand verbundenen Qualitätssicherungsverfahrens. Durch den geringeren organisatorischen Aufwand lässt sich das Qualitätssicherungsverfahren schneller durchführen. Durch die damit verbundene zeitnahe Rückmeldung an die Schlaflaboratorien lassen sich mögliche Qualitätsprobleme schneller beheben.

In der Literatur lassen sich keine vergleichbaren Daten zur Evaluierung der methodischen Durchführung zur Auswahl der Patientendaten in einem Peer-ReviewVerfahren finden. Zwar wurde oft der Einfluss eines Peer-Review-Verfahrens auf die Qualitätsverbesserung untersucht (z. B. McClellan et al. 2004), jedoch nicht die methodische Durchführung bezüglich der Aktenauswahl.

Es lassen sich Beispiele für die Anwendung beider Methoden zur Aktenauswahl im Rahmen eines Peer-Review-Verfahrens in der Literatur finden. Als ein Beispiel für die Anwendung des Stichtagsverfahrens ist das Peer-Review-Verfahren der Gesellschaft für Pneumologie im Projekt zur Qualitätskontrolle in pneumologischen Kliniken zu nennen (Köhler et al. 1998). Patienten, die am vorgegebenen Stichtag stationär aufgenommen wurden und eine pneumologische Erstdiagnose erhielten, nahmen am Verfahren teil. Des Weiteren enthielt dieses Projekt einen Anteil aus dem Randomisierten Verfahren. Um eine „Selektion der Patienten“ zu vermeiden (Köhler et al. 1998, S. 193), wurde zusätzlich eine Aufnahmeliste der Patienten des Stichtags angefordert. Diese erfüllte jedoch nur den Zweck einer Kontrolle. Ein Beispiel für das Randomisierte Verfahren ist bei dem Programm zur Sicherung der Prozessqualität in pneumologischen Praxen zu finden (Fischer et al. 2006). Dabei nahmen am Qualitätssicherungsprogramm Patienten teil, die in der jeweiligen Praxis im definierten Zeitraum mit der Diagnose Asthma bronchiale oder COPD behandelt wurden. Diese Patienten wurden auf einer Patientenliste aufgeführt und aus dieser Patientenliste wurden zufallsmäßig Patientenakten durch die Projektstelle ausgesucht.

Ein weiteres Beispiel für das Randomisierte Verfahren im Peer-Review-Verfahren ist das Qualitätssicherungsverfahren der Deutschen Rentenversicherung. Aus einem Umfang abgeschlossener Rehabilitationen in einem definierten Zeitintervall 
werden zufallsmäßig Patientenakten zur Teilnahme ausgewählt (Dt. Rentenversicherung ... 2007).

Somit lassen sich für beide Methoden Anwendungsbeispiele in der Literatur finden. Die Frage nach welchen Gesichtspunkten die Verantwortlichen eines Qualitätssicherungsprogramms das Konzept zur Aktenauswahl wählen, bleibt an dieser Stelle offen. Die zukünftigen Durchgänge des Qualitätssicherungsprogramms der DGSM werden sich wegen der oben genannten Gründe am Stichtagsverfahren orientieren.

\subsection{Die Qualität in den DGSM-akkreditierten Schlaflaboratorien}

Hauptziel des Qualitätssicherungsverfahrens der DGSM ist die Optimierung der schlafmedizinischen Versorgung in den DGSM-akkreditierten Schlaflaboratorien (DGSM 2011). Bevor Verbesserungen in der Prozessqualität der schlafmedizinischen Zentren vorgenommen werden können, war es notwendig diese zunächst zu erfassen.

Insgesamt konnte zunächst gezeigt werden, dass die meisten Schlaflaboratorien die Anforderungen an eine gute Prozessqualität erfüllen. $91 \%$ (Stichtagsverfahren) bzw. 92 \% (Randomisiertes Verfahren) der teilnehmenden Schlaflaboratorien konnten weiter akkreditiert bleiben. Bei näherer Betrachtung der Einordnung des Gesamtprozesses eines Schlaflabors in Bezug auf den aktuellen Stand wissenschaftlicher Erkenntnisse und Leitlinien lässt sich jedoch darstellen, dass einige schlafmedizinische Zentren offensichtliche Mängel aufweisen. In $36,2 \%$ der am Stichtagsverfahren teilnehmenden Schlaflaboratorien entsprach mindestens eine der drei Patientenakten mit Werten unter 60 auf der Analogskala weniger der wissenschaftlichen Empfehlung und damit nach der Definition von Fischer et al. (2006) einer „schwach ausgeprägten bis defizitären Prozessqualität“. Im Randomisierten Verfahren waren es 37,2\%. Allerdings korreliert die Bewertung des Gesamtprozesses nicht unbedingt mit der Revisitationsentscheidung. Demnach lässt sich ableiten, dass der Gesamtprozess der Patientenversorgung für den Gutachter in den meisten Fällen trotzdem gut und richtig nachvollziehbar war, trotz geringfügig ausgeprägter leitlinienorientierten Behandlung. Wäre dies nicht der Fall gewesen, wäre mit zahlreicheren Revisitationsempfehlungen zu rechnen gewesen. 
Obwohl in den meisten schlafmedizinischen Zentren von einer "guten" bis "durchschnittlichen" Prozessqualität ausgegangen werden kann, zeigten sich in einigen Patientenakten Defizite; der Erfüllungsgrad der meisten Items aus den Beurteilungsfeldern Anamnese, Diagnostik, Diagnosesicherung und Therapie lag zwar sehr hoch, aber es zeigte sich ein besonders hoher fehlender Anteil bestimmter Items. Die meisten "fehlenden" Items betrafen das anamnestische Vorgehen; besonders häufig fehlten in den Patientenakten die Angaben zu „psychischen“ und „vegetativen Beschwerden“, „psychosozialen Belastungen“ und „Risikofaktoren bzw. Suchtanamnese“. Im Schnitt waren diese in 30 \% der Patientenakten der weiterhin akkreditierten Schlaflaboratorien für den Gutachter nicht zu erfassen. In Patientenakten der schlafmedizinischen Zentren, die den Akkreditierungsstatus verlieren, fehlten die Angaben in 59-81\%.

Dass die Einbeziehung des Patienten offensichtlich noch deutlichen Entwicklungsbedarf in der medizinischen Versorgung darstellt, zeigen auch Farin und Jäckel (2011) für das Peer-Review-Verfahren der Deutschen Rentenversicherung in der Rehabilitation. Farin und Jäckel (2011) stellten fest, dass die meisten Defizite in den untersuchten Rehabilitationseinrichtungen bezüglich Prozessmerkmalen vorzufinden waren, die die "Erhebung des Krankheitsverständnisses“ oder die „Selbsteinschätzung des Patienten“ beinhalteten (Farin und Jäckel 2011, S. 179).

Im diagnostischen Vorgehen der akkreditierten Schlaflaboratorien 'fehlten' vor allem Angaben zu "Leistungs- und Vigilanzuntersuchung" (SV $=37,8 \%, R V=40,1$ $\%$ ) und zum "klinischen Labor" (SV = 27,7 \%, RV = $26 \%$ ). In Patientenakten der Schlaflaboratorien, die nicht akkreditiert bleiben, zeigten sich fehlende Werte in den Items „klinisches Labor“ (60,9 \% der Fälle) und „Leistungs- und Vigilanzuntersuchung" (56,5 \% der Fälle).

Warum Angaben zum anamnestischen bzw. diagnostischen Vorgehen häufig fehlten, könnte mehrere Ursachen haben. Die erste wäre sicherlich, dass diese Items tatsächlich nicht vom Patienten erfragt bzw. nicht durchgeführt wurden. Ebenso wäre denkbar, dass die Prozessmerkmale durchgeführt, aber im Entlassungsbericht nicht ausführlich dokumentiert wurden. Fischer et. al (2006) formulieren die Annahme, dass Ärzte davon ausgehen, der weiterbehandelnde Kollege lese aus "Zeitmangel" nur Therapiempfehlungen und daher auf detaillierte Entlassungsberichte verzichtet werden könne (Fischer et al. 2006, S. 490). Bei der Begutachtung 
im Qualitätssicherungsverfahren kann dessen ungeachtet nur das begutachtet werden, was im Entlassungsbericht auch dokumentiert wurde.

Als einen weiteren Grund für die hohe Anzahl an 'fehlend' erfassten Items aus dem Beurteilungsfeld Anamnese könnte man annehmen, dass es am Aufbau der Antwortkategorien des Beurteilungsfeldes liegen könnte. Es enthält nämlich keine Antwortmöglichkeit 'entfällt'. Betrachtet man jedoch die definierten Items, muss man feststellen, dass diese in jedem Fall im Entlassungsbericht dokumentiert werden sollten, da sie das Befinden des Patienten und die Behandlungsnotwendigkeit definieren (z. B. „Allgemeine Anamnese“ oder „organische- und psychische Beschwerden“). Dabei ist es zunächst nicht entscheidend, ob das Schlaflabor diese Angaben selbst erhoben hat oder diese durch vorherige Untersuchungen bereits vorlagen. Entscheidend ist die Dokumentation oder ein kurzer Verweis im Entlassungsbericht, damit der Gutachter die jeweiligen Items ggf. als „extern durchgeführt“ erfassen kann. Erscheint keinerlei Anmerkung im Entlassungsbericht, muss der Gutachter die Items als 'nicht vorhanden' bewerten, sodass man davon ausgehen kann, dass diese vom Schlaflabor tatsächlich nicht erhoben wurden. Jedoch scheinen die häufig 'fehlenden' Items aus dem Beurteilungsfeld Anamnese keinen großen Einfluss auf die Bewertung der Gutachter zu nehmen. Trotz fehlender Items werden nicht automatisch mehr Revisitationsempfehlungen ausgesprochen. Die mangelnde Dokumentation wiederum würde erklären, warum Schlafzentren trotz vieler 'fehlender' Items keine Revisitationsempfehlungen bekommen. Möglicherweise geht der Gutachter bei seiner Bewertung von mangelnder Dokumentation aus und nicht von der tatsächlich 'fehlenden' Durchführung.

Ein anderer möglicher Grund für die häufig fehlenden Angaben vor allem im Beurteilungsfeld Diagnostik, muss an dieser Stelle ebenfalls berücksichtigt werden. In Schlaflaboratorien werden überwiegend schlafbezogene Atmungsstörungen behandelt (110.000 von 126.000 stationär behandelte Patienten 2008 (Statistisches Bundesamt 2009)). In den BUB-Richtlinien (Richtlinien über die Bewertung ärztlicher Untersuchungs- und Behandlungsmethoden) des Gemeinsamen Bundesausschusses aus dem Jahr 2004 wird für die Diagnostik der „obstruktiven Schlafapnoe“ bei Verdacht auf das Vorliegen einer schlafbezogenen Atmungsstörung eine sogenannte 4-Stufendiagnostik vorgegeben (BUB-Richtlinien 2004a). Die erste Diagnosestufe beinhaltet die Anamnese. Die zweite Stufe impliziert die 
klinische Untersuchung zum Ausschluss von endokrinologischen Stoffwechseloder Herz-Kreislauf-Erkrankungen, Ventilationsstörungen sowie neurologische und psychiatrische Erkrankungen. Erst wenn sich nach den diagnostischen Schritten der Verdacht einer schlafbezogenen Atmungsstörung erhärtet, soll zur weiteren Abklärung im Schlaflabor eine kardiorespiratorische Polygraphie (Stufe drei) bzw. kardiorespiratorische Polysomnographie (Stufe vier) eingesetzt werden (BUB-Richtlinien 2004a). Kommt der Patient mit dem Verdacht einer schlafbezogenen Atmungsstörung, sind oft die Stufen eins und zwei bereits durch den Hausarzt durchgeführt worden. Dementsprechend wiederholt das Schlaflabor diese diagnostischen Schritte üblicherweise nicht. Wenn nun der Entlassungsbericht des Schlaflabors verfasst wird, sollte für die überwiegende Mehrzahl der Patientenakten die allgemeine Anamnese, klinische Untersuchung mit Labor und eventuell die kardiorespiratorische Polygraphie schon vorhanden sein. Wird jedoch nicht im Entlassungsbericht auf die bereits erhobenen Vorbefunde verwiesen, wird der Gutachter das Item als 'nicht vorhanden' bewerten. Wurde auf die Vorbefunde jedoch im Entlassungsbericht kurz verwiesen, würde der Gutachter auf „bereits extern durchgeführt" verweisen können. Außerdem hätte der Gutachter in solch einem spezifischen Fall (vierte Stufe BUB-Richtlinien) im Beurteilungsfeld Diagnostik die Möglichkeit von der Antwortkategorie 'entfällt' Gebrauch zu machen. Abgesehen von qualitätssichernden Maßnahmen ist ein ausführlicher Entlassungsbericht in den BUB-Richtlinien vorgeschrieben. Dazu heißt es: „Anamnese, klinische Untersuchungsergebnisse, die Maßnahmen und Ergebnisse der differentialdiagnostischen Abklärung sowie die Ergebnisse der Polygraphie, Polysomnographie, CPAP-Einstellung und aller Therapiekontrollen sind ausführlich zu dokumentieren und dem weiterbehandelnden Arzt zur Verfügung zu stellen" (BUB-Richtlinien 2004b).

Allerdings kann aus der alleinigen Betrachtung der bewerteten Checkliste an dieser Stelle nicht eindeutig entschieden werden, ob sich das Fehlen der Items, die sich auf die Diagnostik beziehen, tatsächlich auf die Nichtdurchführung oder auf eine mangelnde Dokumentation im Entlassungsbericht zurückführen lassen. Möglicherweise entscheidet auch ein eher strenger Gutachter sich bei vielen fehlenden Items eher zu einer Revisitation als ein Gutachter, der vielleicht von Dokumentationsfehlern ausgeht. An dieser Stelle zeigt sich die Limitierung eines Qualitätssi- 
cherungsprogramms bezüglich seiner Aussagekraft in Hinblick auf die Beurteilung der aktuellen Qualität. Festzuhalten bleibt, dass nur das Dokumentierte im Qualitätssicherungsprogramm bewertet werden kann.

In einigen Schlaflaboratorien konnte ein deutliches Defizit in der Erkennung von „Komorbiden Störungen“ abgebildet werden. In 10,1 \% (Stichtagsverfahren) und 8,6 \% (Randomisiertes Verfahren) der Patientenakten der weiterhin akkreditierten Schlafzentren wurden offensichtliche komorbide Störungen (z. B. Depression) anhand der Anamnese und aufgrund durchgeführter Untersuchungen nicht erkannt bzw. nicht dokumentiert. Dies war in $34 \%$ der Patientenakten der nicht mehr akkreditierten Schlaflaboratorien der Fall. Die Schlafmedizin als ein interdisziplinär orientierender Fachbereich setzt voraus, dass der Schlafmediziner Schlafstörungen aufgrund von Interaktionen mit multiplen Erkrankungen aus dem z. B. neurologischen, psychiatrischen oder internistischen Fachbereich erkennen kann. In internationalen Studien konnte gezeigt werden, dass Schlafstörungen häufig mit psychiatrischer Komorbidität einhergehen (Ohayon 2003, Picchietti und Winkelman 2005). Picchietti und Winkelman (2005) zeigten beispielsweise nach einem Vergleich internationaler Studien, dass das gemeinsame Auftreten von Depression und dem Restless-Legs-Syndrom sehr häufig ist. Um so wichtiger ist die ausführliche Darstellung der Schlaf- und Atmungsparameter und vor allem der Beinbewegungen. An dieser Stelle ist in den weiterhin akkreditierten Schlaflaboratorien noch keine zufriedenstellende Qualität der untersuchten Prozessmerkmale erreicht. Dies unterstreicht auch die negative Bewertung der wichtigen zusammenfassenden Parameter. Es konnte ferner gezeigt werden, dass in 14,5 \% (SV) bzw. $11 \%$ (RV) die Beurteilung von „Beinbewegungen“ im Entlassungsbericht 'nicht angemessen' bzw. 'fehlend' dargestellt war (47\% der Patientenakten der nicht mehr akkreditierten Schlaflaboratorien).

An dieser Stelle könnte auch ein Zusammenhang zwischen der nicht erkannten Komorbidität und der 'nicht angemessenen' Beurteilung der Beinbewegungen bestehen. So können auch periodische Beinbewegungen als Komorbidität bei anderen schlafbezogenen Erkrankungen, z. B. dem obstruktiven Schlafapnoesyndrom (OSAS) vorliegen (Rühle und Nilius 2004). Es bleibt festzuhalten, dass in einigen Schlaflaboratorien sowohl pathologische Beinbewegungen als auch andere komorbide Störungen 'nicht angemessen' erkannt wurden. Es besteht dringlicher 
Verbesserungsbedarf. Hierbei geht es in erster Linie darum, ob die medizinische Behandlung des Patienten adäquat durchgeführt wurde. Werden jedoch komorbide Störungen nicht erkannt, findet eine fehlende oder falsche Behandlung des Patienten statt.

Dass die Prozessqualität in den DGSM-akkreditierten Schlaflaboratorien, trotz einiger als fehlend beurteilter Items, auf hohem Niveau zu funktionieren scheint, wurde bei der positiven Beurteilung der "Stimmigkeit des diagnostischen und therapeutischen Konzeptes" deutlich. Ihre Stimmigkeit wird im Großen und Ganzen als 'angemessen' bzw. 'teilweise angemessen' beurteilt ( $\mathrm{SV}=93,3 \%, \mathrm{RV}=95,7$ $\%)$. Unangemessene „Stimmigkeit des diagnostischen bzw. therapeutischen Procedere" bedeute laut der Erläuterungen der Checkliste, dass "die durchgeführten Maßnahmen und Empfehlungen die bisherigen vorliegenden Befunde nicht sinnvoll berücksichtigten und darauf aufbauten. In nur $4 \%$ (SV) und 3,7 \% (RV) der Patientenakten der akkreditierten Schlaflaboratorien erschien das diagnostische Vorgehen 'nicht angemessen'; das therapeutischen Vorgehen erhielt in 4,2 \% (SV) und $4 \%(R V)$ eine unangemessene Beurteilung. In den Patientenakten der Schlaflaboratorien, die den Akkreditierungsstatus verlieren, waren in $22 \%$ (,Stimmigkeit des diagnostischen Procedere“) und $15 \%$ („Stimmigkeit des therapeutischen Procedere") 'nicht angemessen'.

Die vorliegenden Ergebnisse können nicht direkt mit den bereits erfassten Ergebnissen aus dem Qualitätssicherungsprogramm der DGSM 2000 und 2002 verglichen werden, da die jetzt geprüften Prozessmerkmale sich stark zum damaligen Verfahren unterscheiden. Die durchgeführten Durchgänge 2009/10 liefern zunächst einen Status-Quo der existenten Qualität im Schlaflaboratorium.

Es wäre sinnvoll, Ergebnisse anderer Qualitätssicherungsprogramme einzubeziehen. Dies ist prinzipiell nur bedingt möglich, weil die bestehenden Qualitätssicherungsmaßnahmen anderer Fachgebiete auf die selbigen zugeschnitten sind und keine vergleichenden Items vorliegen.

An dieser Stelle kann noch nicht beantwortet werden, ob die qualitätssichernden Maßnahmen der DGSM einen Effekt auf die Verbesserung der Prozessqualität nehmen. Die nächsten Durchgänge der DGSM-Qualitätssicherungsverfahren werden Aufschluss über die Effektivität und Effizienz des Programms liefern. 
In anderen Qualitätssicherungsprogrammen, wie dem Peer-Review-Verfahren der Rentenversicherung, konnten Verbesserungen in ambulanten Einrichtungen verzeichnet und deutliche Qualitätsentwicklungen beobachtet werden (Klosterhuis et al. 2010). Nichtsdestotrotz konnten bei einigen Qualitätsmerkmalen keine positiven Wirkungen erzielt werden. Die erfolgslosen Bemühungen beziehen sich auf die Merkmale im Bereich der „Selbsteinschätzung des Patienten“, sein „Krankheitsverständnis" und die "Kommunikation zwischen Arzt und Patient" (Klosterhuis et al. 2010). Die Erfahrungen und Ergebnisse des Qualitätssicherungsprogramms der Rentenversicherung zeigen, dass zwar deutliche Verbesserungen in der Qualität der Einrichtungen erreicht werden konnten. Trotzdem werden immer noch erhebliche Einrichtungsunterschiede verzeichnet. Schätzungen zufolge weisen nach den Jahren der qualitätssichernden Maßnahmen immer noch 30 \% der Einrichtungen Verbesserungspotenzial auf (Klosterhuis et al. 2010). Positive Wirkungen konnten in Einrichtungen erzielt werden, die von vornherein großes Verbesserungspotential aufwiesen (Jamtvedt et al. 2003). In Einrichtungen, die eine gute Qualität in der Prozessstruktur bewiesen, konnten nur kleine Erfolge erzielt werden (sog. Deckeneffekt).

\subsection{Abhängigkeit der Items in Bezug auf die Gutachterempfeh- lung zur Revisitation}

Es war von Interesse, welche Items ein Gutachter bei der Beurteilung der Prozessqualität im Schlaflabor bei seiner Entscheidungsfindung berücksichtigt. Es war zu prüfen, ob sich Items bestimmen lassen, von denen die Gutachterempfehlung zur Revisitation eines Schlaflabors abhängt.

Im Ergebnis ist es zwar nicht gelungen, einzelne Items zu bestimmen, bei deren Nichterfüllung eindeutig der kausale Zusammenhang mit einer Revisitationsempfehlung mathematisch bewiesen werden konnte. Jedoch ließen sich Items bestimmen, die eine bedeutendere Rolle bei der Entscheidung zur Revisitation zu spielen scheinen als andere.

Die Entscheidung des Gutachters für eine Revisitationsempfehlung scheint zunächst von jedem Item abhängig zu sein. Hohe Signifikanzen $(p \leq 0,05)$ konnten bei den meisten Items aus dem Beurteilungsfeld Entlassungsbericht, beispielsweise bei Items wie "Stimmigkeit des diagnostischen und therapeutischen Procedere" 
ermittelt werden. Items aus dem Beurteilungsfeld Diagnosesicherung ergaben weniger signifikant hohe $p$-Werte.

Bei der Einschätzung der hier vorgestellten Resultate muss aber auch berücksichtigt werden, dass die dem Test zugrundeliegende Fallzahl (447 Patientenakten im Stichtagsverfahren und 354 Patientenakten im Randomisierten Verfahren) sehr groß war. Um die Repräsentativität zu bewahren, konnte die Fallzahl durch zufällige Datenauswahl nicht begrenzt werden.

Aufgrund der ordinal aufgebauten Kategorien der Checkliste (z. B. 'angemessen', 'teilweise angemessen', 'nicht angemessen') konnte zur weiteren Untersuchung dieser Fragestellung keine Faktorenanalyse durchgeführt werden.

Nichtsdestotrotz traten interessante Ergebnisse in Erscheinung, die uns dem Ziel die entscheidenden Items in Bezug auf die Revisitationsempfehlung zu finden, näher bringen. Zum einen konnte festgestellt werden, dass es Items gibt, die nach Meinung der Gutachter besonders häufig in den Patientenakten mit Revisitationsempfehlung für den „spezifischen Fall“ 'fehlten'. Umgekehrt ließ sich erkennen, dass bei einigen fehlenden Items, der Anteil der damit verbundenen Revisitationsempfehlung besonders hoch lag. Besonders ausgeprägt zeigte sich dies bei Items aus dem Beurteilungsfeld Entlassungsbericht, die bezüglich ihrer Angemessenheit bewertet wurden. $67 \%(\mathrm{~N}=48)$ der Patientenakten, in denen das Item "Stimmigkeit des diagnostischen Procedere" als 'nicht angemessen' beurteilt wurde, erhielten eine Revisitationsempfehlung. 60,5\% ( $\mathrm{N}=43)$ der Akten, in der die "Stimmigkeit des therapeutischen Procedere" vom Gutachter als 'fehlend' bzw. 'nicht angemessen' beurteilt wurde, erhielten ebenfalls eine Revisitationsempfehlung.

Das Item „Stimmigkeit des diagnostischen Procedere“ wurde in den Patientenakten mit Revisitationsempfehlung im Vergleich zu Akten ohne Revisitationsentscheidung 15-mal (30 \% zu $2 \%$ ) häufiger als 'nicht angemessen' vom Gutachter beurteilt. Das Item "Stimmigkeit des therapeutischen Procedere" wurde 8-mal häufiger ( 25 zu 3 \% $)$ in den Patientenakten mit Revisitationsempfehlung als 'nicht angemessen' beurteilt im Vergleich zu Patientenakten ohne Empfehlung zur Revisitation. Obwohl diese Erkenntnis auf einer niedrigen Fallzahl $(\mathrm{N}=48$ bzw. $N=43)$ der betreffenden Patientenakten basiert, 
zeigt sich mehr als eindrücklich der Zusammenhang zwischen einem als 'nicht angemessen' beurteilten Item und einer Revisitationsempfehlung.

Des Weiteren erschien die Gutachterempfehlung zur Revisitation von Items wie „Spezifische Anamnese“ und „Komorbide Störungen erkannt“ in höherem Maße abzuhängen. Patientenakten, in denen Angaben zur „Spezifischen Anamnese“ 'fehlten' und „komorbide Störungen“ 'nicht erkannt' wurden, erhielten besonders häufig eine Revisitationsempfehlung („Spezifische Anamnese Tag“ (51,5 \%), „Nacht“ (46,3\%), „Komorbide Störungen nicht erkannt“ (43 \%)).

Darüber hinaus fallen eine Vielzahl von Items auf, deren Bedeutung bei der Entscheidung zur Revisitation ungewiss erscheinen. In den Patientenakten, in denen z. B. Angaben zu „organischen, psychischen, vegetativen Beschwerden“ nach Meinung des Gutachters 'nicht vorhanden' waren, haben zwischen 68,1\% und $75,5 \%$ keine Revisitationsempfehlung erhalten. Des Weiteren zeigte sich, dass es trotz vorhandener Items Revisitationsempfehlungen gibt.

Obwohl das alleinige Vorliegen bzw. Fehlen einzelner Items keinen großen Einfluss auf die Entscheidung des Gutachters bezüglich einer Revisitationsempfehlung nimmt, scheinen Items, mit der sich Gesamtbehandlungsverläufe beurteilen lassen, wie „Stimmigkeit des diagnostischen und therapeutischen Procedere“, „Zusammenfassende Beurteilungen“ und „Komorbide Störungen erkannt“" wesentlicher für den Entscheidungsprozess der Gutachter zu sein.

Dazu kann angemerkt werden, dass diese Items mehrere Items aus den unterschiedlichen Beurteilungsfeldern Anamnese, Diagnostik und Diagnosesicherung beinhalten. So bedingt möglicherweise bereits eine 'nicht vorhandene' „Spezifische Anamnese“, oder eine 'nicht angemessene' Beurteilung der diagnostisch-erfassten Parameter das Nichterkennen „komorbider Störungen“ und damit entsprechende Folgefehler in der Diagnose und Therapieempfehlung.

Dass Gutachter nicht zwangsläufig nur auf das Vorliegen bestimmter, im Entlassungsbericht dokumentierter, Items Wert legen, wird auch gestützt durch die Gegenüberstellung des Erfüllungsgrades einzelner Items in den beiden durchgeführten Verfahren. Im Vergleich beider Verfahren zeigte sich, dass der Anteil dokumentierter Items aus dem Beurteilungsfeld Anamnese und Diagnostik im 
Stichtagsverfahren in den akkreditiert bleibenden Schlaflaboratorien zwar zum Teil deutlich niedriger als im Randomisierten Verfahren lag. Auf die Beurteilung des Items "Stimmigkeit des diagnostischen Procedere“ schien es trotzdem keinen Einfluss zu nehmen. In beiden Verfahren wurde diese in $4 \%$ der Patientenakten als 'nicht angemessen' beurteilt.

Der Gutachter bewertet also nicht nur, ob das Item im Entlassungsbericht dokumentiert ist. Er prüft vor allem, ob die Angaben in den Gesamtbehandlungsverlauf richtig eingeordnet wurden. Diese zentrale Aussage der Arbeit widerspricht dem oft geäußerten Kritikpunkt bezüglich Peer-Review-Verfahren, dass durch „inhaltlich standardisierte Floskeln“ im Entlassungsbericht manipuliert werden könnte (Klosterhuis 2008, S. 262). Wie Kutschmann (2004) bereits feststellte, lässt sich das "Schonfärben" von Akten nicht gänzlich abwenden. Dieses "Schönfärben“ scheint insgesamt wenig Einfluss auf die endgültige Entscheidung bzw. Bewertung zu nehmen. Natürlich benötigt der behandelnde Arzt/in die Angaben zur „Allgemeinen Anamnese" und "Organischen Beschwerden“. Das alleinige Wissen darüber befähigt inn allerdings nicht automatisch, diese Informationen im Sinne der Weiterbehandlung richtig einzuordnen. Den Anspruch auf qualitative Einordnung erfasst ein übergeordnetes Item wie die „Stimmigkeit des diagnostischen und therapeutischen Procedere“. Diese Voraussetzung wird in folgender Weise erfüllt: Indem der Gutachter die „Stimmigkeit“ des Procedere beurteilt, wird ein qualitative Aussage gemacht. Es wird im Gegensatz zu anderen Items, die sich ausschließlich auf das Vorhandensein beziehen, über die quantitative Ebene hinausgegangen.

Dass die Beurteilung des Erfüllungsgrades einzelner Items trotzdem einen Teil des Konzepts anderer Peer-Review-Verfahrens darstellt, wird anhand des vergleichbaren Peer-Review-Verfahrens der Deutschen Rentenversicherung deutlich. Darin findet man diesbezüglich in den Beurteilungskriterien, dass die einzelnen qualitätsrelevanten Prozessmerkmale und die zusammenfassenden Bewertungen sowohl „quantitative (Anzahl erfüllter Kriterien bzw. Merkmale) als auch qualitative Gesichtspunkte (Gewichtung einzelner Kriterien bzw. Merkmale je nach den spezifischen Gegebenheiten des jeweiligen Falls)" berücksichtigen sollen (Lindow et al. 2007, S. 11). 
Mit dieser Forderung kommt zusätzlich ein interessanter Aspekt zum Vorschein, der an dieser Stelle diskutiert werden soll: die Subjektivität des Gutachters in einem Peer-Review-Verfahren.

Ist die Erkenntnis, dass mittels statistischer Verfahren keine eindeutigen Ergebnisse bezüglich der Items bestimmt werden konnten, damit in Zusammenhang zu bringen, dass die Gutachter nur nach subjektiven Gesichtspunkten ihre Entscheidung zur Revisitationsempfehlung fällen? Kann deshalb eine statistische Auswertung keine genauen Zahlen liefern?

In der vorliegenden Arbeit wurden die 25 an beiden Verfahren teilnehmenden Gutachter im Hinblick auf die Anzahl der Revisitationsempfehlungen gegenüberstehend verglichen. Gutachter, die im Stichtagsverfahren viele Revisitationsempfehlungen aussprachen, und somit als tendenziell streng angesehen werden könnten, taten dies nicht ebenso beim Randomisierten Verfahren. Nichtsdestotrotz lässt sich ein Unterschied in der Urteilsstrenge der Gutachter damit nicht ausschließen. Wie auch Kutschmann (2004) bereits angibt, minimiert die kombinierte Beurteilung durch mehrere Gutachter die Subjektivität eines einzelnen Gutachters. Zur Gewährleistung der Übereinstimmungen von Bewertungen durch die Gutachter im Peer-Review-Verfahren sei es laut Jäckel et al. (1997) generell notwendig, für die Übereinstimmung der Beurteilungsmaßstäbe der Gutachter zu sorgen. Im DGSM-Qualitätssicherungsprogramm sollen die Beurteilungshinweise im Manual dem Gutachter bei der Bewertung helfen (Kutschmann 2004).

Nutzt man die Erkenntnisse von Kutschmann (2004) zur Untersuchung der Gutachterreliabilität im DGSM-Verfahren 2000 und 2002 mit damals zum Teil gleichen und zusätzlich mit vergleichbaren Items, so unterstreichen diese die Hypothese, dass die Entscheidung zur Revisitation u. a. stark von der Subjektivität der Gutachter abzuhängen scheint trotz der gegebenen Beurteilungshinweise. Für das DGSM-Peer-Review-Verfahren 2000 und 2002 bewertet Kutschmann in seiner Untersuchung der Interrater-Reliabilität das Beurteilungsfeld Anamnese mit „weniger zufriedenstellend“, Diagnostik als, „zufriedenstellend“ und Therapie ebenfalls als „zufriedenstellend“ (Kutschmann 2004, S. 124). Dies zeigt zwar eine befriedigende Leistung des Programms, kann jedoch auch kritisch hinterfragt werden, zumal die Intrarater-Reliabilität für das Beurteilungsfeld Anamnese als „zufriedenstellend“, für Diagnostik „gut“ und Therapie „zufriedenstellend“ von Kutschmann 
eingestuft wurde (Kutschmann 2004, S. 124). Daher sei die Subjektivität laut Kutschmann (2004) nicht nur jedes einzelnen Gutachters variabel, sondern jeder Gutachter selbst bewertet nicht immer gleich. Als Beobachtung formulierte er, dass die Gutachterreliabilität oft dann eher gering sei, wenn in den Beurteilungshinweisen im Manual Formulierungen wie „nach spezifischen Gegebenheiten des jeweiligen Falls“ oder „nach der Lage des Falls“ verwendet wurden. Damit würden eindeutige Anhaltspunkte fehlen, an welcher Stelle einzelne Prozessabläufe als 'vollständig', 'teilweise' oder 'nicht angemessen' zu bewerten seien (Kutschmann 2004, S. 128). Diese Formulierungen finden sich im aktuellen Verfahren in allen Beurteilungsfeldern, außer dem Beurteilungsfeld Anamnese. Im Beurteilungsfeld Entlassungsbericht findet sich der Hinweis, der Begriff 'angemessen' solle ,je nach spezifischem Fall durch die jeweiligen Gutachter spezifiziert“ werden. Das Item „Stimmigkeit des diagnostischen Procedere“, welches besonderes Gewicht bei der Beurteilung besitzt, soll danach beurteilt werden, „ob durchgeführte diagnostische Maßnahmen [...] die bisherigen bekannten Befunde sinnvoll berücksichtigen und darauf aufbauen“. Dieses Beispiel zeigt eindrucksvoll, wie die Beurteilung einiger Items fast vollständig auf der Meinung des Gutachters basieren können. Wie Kutschmann (2004) bereits feststellte, lässt sich die hier erörterte Subjektivität der Gutachter zukünftig nur sehr schwer vermeiden. Allerdings ist die Berücksichtigung des „spezifischen Falls“ bei der Bewertung insbesondere interdisziplinärer Fächer wie der Schlafmedizin notwendig. Jeder Gutachter wird vermutlich aufgrund seiner Erfahrungen und Überzeugung diese Erfordernisse anders bewerten. Wie Kutschmann (2004) ebenfalls konstatierte, komme noch hinzu, dass der Gutachter den jeweiligen Patienten nicht selbst behandelt hat und den Fall nur anhand der Aktenlage beurteilen kann. Somit müsse er die Bewertung 'angemessen' in eigenem Ermessen treffen.

Ein anderer Aspekt kommt in der Subjektivitätsbeurteilung erschwerend hinzu. Die Gutachter können anhand des Briefkopfes der Entlassungsberichte diese den einzelnen Kliniken bzw. Praxen zuordnen. Dadurch lässt sich keine vollständige Objektivität erzielen, jedoch ist hier auf die Professionalität der Gutachter zu vertrauen.

Um eine möglichst objektive Begutachtung zu gewährleisten, werden in anderen Peer-Review-Verfahren, wie z. B. im Verfahren der Rentenversicherung, Schulun- 
gen der Gutachter durchgeführt (Klosterhuis 2010). Im DGSM-Verfahren wurde auf die Schulung der Gutachter verzichtet, da man - wie bereits im Qualitätssicherungsverfahren 2000 und 2002 - auf die Berufserfahrung der Gutachter vertraute (Kutschmann 2004). Jedoch lässt sich nicht leugnen, dass die Subjektivität in der Beurteilung der Patientenakten eine größere Rolle spielt als angenommen wird. Es muss geprüft werden, ob Schulungen der Gutachter die Objektivität fördern können.

Ein anderer Weg, die Subjektivität in der Entscheidungsfindung des Gutachters im Peer-Review-Verfahren zu minimieren, könnten Handlungsempfehlungen in Form von Leitlinien darstellen. Die DGSM hat im Jahr 2001 die S2-Leitlinie zum „Nicht erholsamen Schlaf“ publiziert (Fischer et al. 2001). 2009 folgte die S3-Leitlinie zum selbigen Thema (Mayer et al. 2009). Jeder Gutachter und jedes Labor hat hierzu Zugang. An dieser Stelle ist erneut auf die Interdisziplinarität der Schlafmedizin einzugehen. Zwar wird in den herausgegebenen Leitlinien das diagnostische und therapeutische Vorgehen für jede schlafmedizinische Erkrankung empfohlen. In der Schlafmedizin als interdisziplinärem Fachgebiet müssen im Schlaflabor Erkrankungen aus dem Gebiet der Neurologie, Psychiatrie oder auch Innere Medizin erst als solche erkannt werden, um eine adäquate Behandlung zu erfahren.

Zusammenfassend lässt sich konstatieren, dass die Gutachterempfehlung zur Revisitation nicht von einzelnen Prozessmerkmalen abhängt. Weder führten positive Bewertungen einzelner Items zwangsläufig zur Nichtrevisitation, noch konnte eine negative Bewertung zwangsläufig mit einer Revisitation in Verbindung gebracht werden. Der Schluss liegt nahe, dass erst ein Zusammenspiel mehrerer negativ bewerteter Items einen Gutachter zur Revisitationsentscheidung bewegt. Diese Tatsache wird gestützt durch die Beobachtung, dass Items, die Gesamtverläufe beurteilen lassen (z. B. „Stimmigkeit des diagnostischen und therapeutischen Procedere“ oder „Komorbide Störungen erkannt“), einen rechnerisch größeren Einfluss auf die Gutachterentscheidung zu nehmen scheinen. Diese Feststellung führt aber nicht automatisch dazu, dass auf die quantitative Bestimmung einzelner Items ('vorhanden'/'nicht vorhanden') verzichtet werden kann. Das Ziel der Qualitätssicherung ist im ersten Schritt die Erfassung, langfristig aber die Verbesserung der Qualität. Dazu ist es wichtig, die Prozesse zu erkennen, in denen Handlungs- 
bedarf besteht. Dies kann nur erreicht werden, wenn einzelne Schritte im Prozess bewertet werden.

Die Frage, welche Items sich als Qualitätsindikatoren von Prozessmerkmalen in interdisziplinären Fachgebieten eignen, kann nach den bisher erworbenen Erkenntnissen folgendermaßen beantwortet werden: in einem interdisziplinären Fachgebiet wie der Schlafmedizin sind Qualitätsindikatoren notwendig, die eine umfassende Beurteilung verschiedener Erkrankungen zulassen. Schlafstörungen können aufgrund von Interaktionen mit multiplen Erkrankungen aus dem neurologischen, psychiatrischen, internistischen, HNO und zahnärztlichen Fachbereichen assoziiert sein. Die Items müssen all diese Bereiche abdecken bzw. flexibel einsetzbar sein. Dafür scheinen übergeordnete Items wie z. B. „Stimmigkeit des diagnostischen Procedere“ und "Stimmigkeit des therapeutischen Procedere“ oder „Komorbide Störungen erkannt“ eine Möglichkeit zu sein. Die Untersuchungen in der vorliegenden Arbeit haben gezeigt, dass die Gutachterentscheidung von solchen Items in hohem Maße abhängt. Auf der anderen Seite sind solche Items aber sehr variabel und anfällig für die Subjektivität der Gutachter, der sehr komplexe Sachverhalte in die Beurteilung solcher Items mit einbeziehen muss.

\subsection{Methodenkritik}

Wie bei jeder wissenschaftlichen Untersuchung sind auch bei den Erhebungen und Auswertungen im DGSM-Qualitätssicherungsverfahren bestimmte Einschränkungen zu berücksichtigen, die die Aussagekraft der Ergebnisse begrenzen.

Bezogen auf die Durchführung des Qualitätssicherungsprogramms fallen folgende Einschränkungen auf:

- die Auswahl der Items für die Checkliste im DGSM-Qualitätssicherungsverfahren basiert auf Expertenmeinungen. Dies stellt eine niedrige Evidenzstufe dar und kann damit als ein limitierender Faktor angesehen werden.

- die Bewertung der Checkliste basiert in den Beurteilungsfeldern Anamnese, Diagnostik, Therapie lediglich auf dem Erfüllungsgrad der einzelnen Items. Die Checkliste ist darauf ausgelegt nur das Vorhandensein der jeweiligen Items zu erfassen. Es wird nicht in Gänze erfasst, wie die Qualität der durchgeführten Items tatsächlich ist. 
Vergleicht man diesbezüglich das DGSM-Qualitätssicherungsverfahren mit anderen Peer-Review-Verfahren, so fällt ein entscheidender Unterschied im Aufbau der Bewertungskategorien auf. Im Reha-Qualitätssicherungsprogramm der Deutschen Rentenversicherung z. B. wird nicht nur das Vorhandensein der Items gemessen, sondern auch ob diese Items Mängel aufweisen oder nicht. Die Bewertungskategorien beinhalten die Abstufung von „keine Qualitätsmängel, leichte und gravierende Qualitätsmängel“" (Lindow et al. 2007, S. 11).

Ein anderer limitierender Faktor in der methodischen Durchführung ist die alleinige Bewertung der Patientenakte anhand eines Entlassungsberichtes des Patienten. Dem Gutachter stehen weder Vorbefunde noch Therapiepläne zur Verfügung. Eine umfassende Beurteilung der durchgeführten Behandlung des Patienten ist damit nur begrenzt möglich. Laut Kutschmann stellen vollständige Patientenakten inklusive der Behandlungs- und Therapiepläne die optimale Informationsquelle zur Beurteilung der Prozessqualität dar (Kutschmann 2004, S. 107). Dennoch hat sich die Bewertung anhand des Entlassungsberichtes in den letzten Jahren in vielen Qualitätssicherungsprogrammen durchgesetzt.

Es stellt sich die Frage, ob bei drei Patientenakten pro Schlaflabor die Repräsentativität der Stichproben insgesamt gegeben ist. Eine größere Stichprobe würde mit einem erhöhten Aufwand einhergehen, das wiederum würde zur zeitlichen Verzögerung und damit zu einer sehr späten Rückmeldung an die Schlaflaboratorien führen. Insgesamt dauerte die Erfassung und Auswertung der Patientenakten im Randomisierten Verfahren schon so lange, dass eine zeitnahe Rückmeldung an die Schlafzentren fast nicht vorgenommen werden konnte. Der Aspekt einer zügigen Rückmeldung erscheint an dieser Stelle eine wichtige Rolle zu spielen, sodass die Stichprobe auf drei Patientenakten einzugrenzen bleibt.

Des Weiteren ergeben sich einschränkende Faktoren in der Auswertung der erfassten Daten.

Die Untersuchung der einzelnen Fragestellung in der vorliegenden Arbeit konnte nur anhand der von den Gutachtern beurteilten Checkliste vorgenommen werden. Daraus war weder die Diagnose des Patienten, noch die Besonderheiten im Stand des diagnostischen Geschehens (BUB-Richtlinien) ersichtlich. Dementsprechend war die Beurteilung einiger als 'nicht vorhanden' erfassten Items nicht genau nachvollziehbar. Es konnte nicht beurteilt werden, aus welchem Grund das Item 
als 'nicht vorhanden' eingestuft wurde. Fehlte die Dokumentation, weil es tatsächlich nicht durchgeführt wurde oder fehlte es, weil eine wiederholte Durchführung entfiel (z. B. in Stufe 4 nach den BUB-Richtlinien)? Möglicherweise fehlte das Item zwar oft im Entlassungsbericht, obwohl es in der Vorbehandlung schon durchgeführt wurde. Dadurch konnte nur indirekt auf die Qualität der Schlaflaboratorien geschlossen werden.

Bei der statistischen Auswertung (Chi ${ }^{2}$-Test) zur Beantwortung der Frage, ob sich einzelne Items bestimmen lassen können, von denen die Gutachterempfehlung zur Revisitationsempfehlung abhängen, muss erwähnt werden, dass die Fallzahl (Patientenakten) sehr hoch war. Je größer die Fallzahl ist, um so mehr ist eine Signifikanz zu erwarten. Dennoch wurde der $\mathrm{Chi}^{2}$ - Test benutzt um der Fragestellung nachzugehen. Da kein metrischer Datentyp vorliegt, konnte keine Faktorenanalyse zur Untersuchung dieser Fragestellung durchgeführt werden. Diese wäre zur Beantwortung der Aufgabenstellung wünschenswert gewesen.

\subsection{Ausblick}

Nach den vorliegenden Ergebnissen ist für die nächsten Durchgänge des Qualitätssicherungsverfahrens der DGSM das Stichtagsverfahren zur Erhebung der Patientenakten zu empfehlen.

Es konnte die aktuelle Prozessqualität der DGSM-akkreditierten Schlaflaboratorien erfasst werden. Ob und in welchem Ausmaß sich Verbesserungen in der Prozessqualität der Schlaflaboratorien ergeben, sollte in den nächsten Jahren weiter untersucht werden.

Bestehende qualitätsrelevante Prozessmerkmale (Items) der Checkliste im DGSM-Qualitätssicherungsverfahren sollten immer wieder auf die Eignung für die Abbildung der Prozessqualität in Schlaflaboratorien überprüft werden. Vielleicht konnte die vorliegende Arbeit einen Beitrag zur Weiterentwicklung von neuen Items für interdisziplinäre Fachgebiete am Beispiel des interdisziplinären Fachbereichs der Schlafmedizin leisten.

Weitere Untersuchungen zur Eignung der Qualitätsindikatoren von Prozessmerkmalen in interdisziplinären Fachgebieten wie der Schlafmedizin wären wünschenswert. Beispielsweise könnte erfasst werden, ob die Gutachterempfehlung zur Revisitation von einer gewissen Anzahl als „fehlend“ erfasster Items oder einer 
bestimmten Kombinationen negativ beurteilter Items abhängen. Mittels Befragung der Gutachter könnte man dazu Erkenntnisse gewinnen oder mittels statistischer Untersuchungen Kombinationen einzelner Items analysieren.

Erstrebenswert wäre es, die Objektivität der Gutachter zu erhöhen. Denkbar ist an dieser Stelle die Einführung eines Punktescores für jedes einzelne Item. Den Items, die für eine gute Prozessqualität notwendig sind, wird der höchste Wert zugeteilt. Weniger entscheidende Prozessmerkmale erhalten einen niedrigen Wert. Ein Ergebnisbereich wird definiert, der zu einer Revisitation führt oder nicht. Die Anwendbarkeit solcher Punkteverfahren in interdisziplinären Fachbereichen müsste zunächst näher überprüft werden. Es wäre aber möglicherweise ein Mittel, um die Subjektivität des Gutachters zu minimieren. Des Weiteren könnte über Schulungen der Gutachter nachgedacht werden. 


\section{Zusammenfassung}

Das Bemühen um eine optimale medizinische Versorgungsqualität, der Wunsch nach Transparenz in der Medizin und knappe finanzielle Ressourcen rücken das Interesse an qualitätssichernden Maßnahmen in den Mittelpunkt. Zwar schreibt die Gesundheitspolitik einerseits Qualitätssicherung auf verschiedenen Ebenen vor, andererseits fehlen entsprechende Organisationsstrukturen und vor allem inhaltliche Vorgaben müssen noch erarbeitet werden.

Die Deutsche Gesellschaft für Schlafforschung und Schlafmedizin (DGSM) führte im Jahr 2009 und 2010 ein Qualitätssicherungsprogramm zur Überprüfung von Prozessqualität in den akkreditierten Schlaflaboratorien als verpflichtenden Bestandteil der Weiterakkreditierung der schlafmedizinischen Zentren durch. In einem Peer-Review-Verfahren bewerteten drei Gutachter anhand jeweils einer Patientenakte, bestehend aus dem Entlassungsbericht und dem Nachtprotokoll, mit Hilfe einer sogenannten "Checkliste qualitätsrelevanter Prozessmerkmale" die Prozessqualität in den DGSM-akkreditierten Schlaflaboratorien. Zur Auswahl der Patientenakten wurden jeweils zwei unterschiedliche Verfahren angewendet: das sogenannte Stichtagsverfahren und das Randomisierte Verfahren.

Im Stichtagsverfahren wurde dem Schlaflaboratorium ein Stichtag angegeben. Die drei Patientenakten, die nach diesem Stichtag erstellt wurden, nahmen am Qualitätssicherungsverfahren teil. Im Randomisierten Verfahren hatten die Schlaflaboratorien für einen definierten Zeitraum die behandelten Patienten auf einer Patientenliste aufzuführen. Zufallsmäßig wurde daraus durch die Verantwortlichen für das qualitssichernde Verfahren drei Patientenakten ausgewählt. Der Akkreditierungsstatus des Schlaflaboratoriums wurde aufgehoben, wenn mindestens zwei Gutachter unabhängig voneinander aufgrund minderer Prozessqualität eine Revisitation empfohlen haben.

Die vorliegende Arbeit sollte auf Grundlage der beiden abgeschlossenen Durchgänge folgende Fragen beantworten:

1. Zu welchen Ergebnissen kommen die angewandten methodischen Verfahren hinsichtlich des Organisationsablaufs und welche Vor- und Nachteile haben sie im Vergleich? Einschließlich der Prüfung, ob das methodische Verfahren einen Ein- 
fluss auf den Ausgang des Ergebnisses bezüglich der Akkreditierung bzw. auf das Verhalten des Gutachters bezüglich Revisitationsempfehlung hat?

2. Wie ist die Qualität der durch die DGSM akkreditierten teilnehmenden Schlafzentren, welche Mängel in den Beurteilungsfeldern sind offensichtlich und wie ist die Auswirkung auf die Weiterakkreditierung?

3. Lassen sich Items bestimmen, von denen die Gutachterempfehlungen zur Revisitation in hohem Maße abhängen, d. h. welche Items eignen sich als Qualitätsindikatoren von Prozessmerkmalen in interdisziplinären Fachgebieten?

Es konnte gezeigt werden, dass das Stichtagsverfahren eher den Ansprüchen genügt, ein unkompliziertes und mit weniger zusätzlichem Aufwand verbundenes Qualitätssicherungsverfahren zu sein. Ein Zusammenhang zwischen dem Ergebnis der Weiterakkreditierung der Schlaflaboratorien und dem methodischen Vorgehen konnte nicht festgestellt werden.

$91 \%$ der teilnehmenden Schlaflaboratorien im Stichtagsverfahren und $92 \%$ der teilnehmenden Schlafzentren konnten weiter akkreditiert bleiben. Insgesamt kann von einer guten Prozessqualität in den DGSM-akkreditierten Schlaflaboratorien ausgegangen werden; das diagnostische und therapeutische Vorgehen wurde nur in weniger als $5 \%$ der Patientenakten als 'nicht angemessen' beurteilt. Verbesserungsbedarf besteht bei Prozessmerkmalen, die sich am Patientenbefinden orientieren (z. B. ein hoher als 'nicht vorhanden' erfasster Anteil von „Organischen, Psychischen, Vegetativen Beschwerden“).

Es konnten keine einzelnen Items bestimmt werden, von denen die Gutachterempfehlung zur Revisitation in hohem Maße abhängen. Positive Bewertungen einzelner Items führten nicht zwangsläufig zur Nichtrevisitation. Jedoch konnte gezeigt werden, dass in Patientenakten, in denen die Items „Stimmigkeit des diagnostischen und therapeutischen Procedere" oder "Komorbide Störungen erkannt" eine negative Beurteilung erfahren haben, besonders oft mit einer Revisitationsempfehlung einhergingen. Diese Feststellung lässt die These zu, dass diese Prozessmerkmale für die Gutachterentscheidung von großer Bedeutung sind.

Diese übergeordneten, verschieden definierbaren Prozessmerkmale sind es auch, die in interdisziplinären Fachgebieten wie der Schlafmedizin als Qualitätsindikatoren eingesetzt werden sollten. 


\section{$7 \quad$ Literaturverzeichnis}

AASM (American Academy of Sleep Medicine): International classification of sleep disorders, 2nd ed: Diagnostic and Coding Manual. American Academy of Sleep Medicine, Westchester/lllinois 2005

AWMF (Arbeitsgemeinschaft der Wissenschaftlichen Medizinischen Fachgesellschaften): Leitlinien-Glossar: Begrifflichkeiten und Kommentare zum Programm für Nationale Versorgungsleitlinien. (Schriftenreihe, Band 30), hrsg. v. Arbeitsgemeinschaft der Wissenschaftlichen Medizinischen Fachgesellschaften/Ärztliches Zentrum für Qualität in der Medizin, Berlin 2007 verfügbar: http://www.awmf.org/fileadmin/user_upload/Leitlinien/Werkzeuge/llglossar.pdf (abgerufen: 12.12.2011)

Blumenstock G (2011): Zur Qualität von Qualitätsindikatoren. Bundesgesundheitsblatt $\underline{54}$, 154-159

Bollschweiler E: Nationale und klinikinterne Leitlinien: Definition und Problemlage; in: Gesundheitsökonomie, Qualitätsmanagement und Evidence-based Medicine, hrsg. v. Lauterbach KW, Schrappe M u. a., Schattauer GmbH, Stuttgart 2001, 457-463

BQS-Institut für Qualität und Patientensicherheit: BQS Qualitätsreport 2008: KnieTotalendoprothese, BQS Qualitätsindikatoren; BQS, o. O. 2008 verfügbar: http://www.bqs-qualitaetsreport.de/2008/ergebnisse/leistungsbereiche/ knietotalerst/uebersicht (abgerufen: 13.03.2012)

BUB-Richtlinien (2004a) = BUB-Richtlinien-Text der Bekanntmachung im Bundesanzeiger Nr. 213 (S. 22 699) vom 10. November 2004, § 3 Stufendiagnostik

BUB-Richtlinien (2004b) = BUB-Richtlinien-Text der Bekanntmachung im Bundesanzeiger Nr. 213 (S. 22 699) vom 10. November 2004, § 6 Dokumentation

Congress of the European Sleep Resarch Society, 14.-18. Sept 2010, Lissabon (Poster)

DGSM (2007) = Die Deutsche Gesellschaft für Schlafforschung und Schlafmedizin verfügbar: http://www.charite.de/dgsm/dgsm/dgsm.php?language=german (abgerufen 14.01.2011)

$\operatorname{DGSM}(2009 a)=$ Qualitätssicherung und Akkreditierung. Qualifikationsnachweis Somnologie. Qualitätsmanagement in der Schlafmedizin. QN für Ärzte: Qualifikationsnachweis (QN) „Somnologie“ für Ärzte (letzte Aktualisierung: 22.09.2009). verfügbar: http://www.charite.de/dgsm/dgsm/akkreditierung_somnologie_qnaerzte.php (abgerufen: 12.03.2010) 
$\operatorname{DGSM}(2009 b)=$ Qualitätssicherung und Akkreditierung. Akkreditierung von Schlaflaboren. download: Fragebogen zur Begutachtung des Schlaflabors (letzte Aktualisierung: 27.02.2009) verfügbar: http://www.charite.de/dgsm/dgsm/akkreditierung_akkreditierung.php? language=german (abgerufen: 16.02.2011)

DGSM (2009c) = Qualitätssicherung und Akkreditierung. Qualitätssicherung Ergebnisqualität: Projekt zur Sicherung der Ergebnisqualität im akkreditierten Schlaflabor (letzte Aktualisierung 26.02.2009) verfügbar: http://www.charite.de/dgsm/dgsm/akkreditierung_qualitaetssicherung_ ergebnisqualitaet.php?language=german (abgerufen: 16.02.2011)

DGSM (2011) = Qualitätssicherung und Akkreditierung. Qualitätssicherung in der Schlafmedizin. Prozessqualität: Qualitätssicherung in DGSM- akkreditierten Schlaflabor: Peer-Review-Verfahren zur Sicherung der Prozessqualität- Aktuelles Qualitätssicherungsverfahren (Durchführung seit 2009) (letzte Aktualisierung 1.04.2011) verfügbar: http://www.charite.de/dgsm/dgsm/akkreditierung_qualitaetssicherung_ prozessqualitaet.php?language=german (abgerufen: 17.07.2011)

DIN EN ISO 8402 (Deutsches Institut für Normung): Qualitätsmanagement-Begriffe. Beuth Verlag, Berlin 1995

DIN EN ISO 9000 (Deutsches Institut für Normung): QualitätsmanagementsystemeGrundlagen und Begriffe. Beuth Verlag, Berlin 2000

Dt. Rentenversicherung-Sozialmedizin und Forschung (2007): Reha-Qualitätssicherung. Peer-Review. download: Kurzbeschreibung Peer-Review-Verfahren (letzte Aktualisierung 24.11.10) verfügbar: http://www.deutsche-rentenversicherung.de/cae/servlet/contentblob/ 36036/publicationFile/2080/kurzinfo_peer_review.pdf (abgerufen: 17.11.2011)

Farin E, Jäckel WH (2011): Qualitätssicherung und Qualitätsmanagement in der medizinischen Rehabilitation. Bundesgesundheitsblatt $\underline{54}, 176-184$

Fischer J, Raschke F, Kutschmann M (1999): Die Checkliste qualitätsrelevanter Prozessmerkmale für das Peer-Review-Verfahren der Deutschen Gesellschaft für Schlafforschung und Schlafmedizin (DGSM) zur Sicherung der Prozessqualität im akkreditierten Schlaflabor. Somnologie $\underline{3}, 335-346$

Fischer J, Mayer G, Peter HJ, Riemann D, Sitter H (2001): Leitlinie „S2“ der Deutschen Gesellschaft für Schlafforschung und Schlafmedizin (DGSM): Nicht-erholsamer Schlaf. Somnologie $\underline{5(3)}, 1-258$ 
Fischer J, Köhler D, Magnussen H (2006): Sicherung der Prozessqualität in der pneumologischen Praxis mittel Peer-Review-Verfahren - ein Pilotprojekt. Pneumologie $\underline{60}, 485-492$

Geraedts M, Selbmann HK, Ollenschlaeger (2003): Critical appraisal of clinical performance meausures in Germany. Int J Qual Health Care 15(1), 79-85

Glattacker M, Jäckel WH (2007): Evaluation der Qualitätssicherung-aktuelle Datenlage und Konsequenzen für die Forschung. Das Gesundheitswesen $\underline{69}$ (5), 277-283

Grimm J, Grimm W: Deutsches Wörterbuch von Jacob und Wilhelm Grimm, Bd. 13. Deutscher Taschenbuch Verlag, München 1984

HistWbPhilos (1989) = Blasche S: Qualität; in: Historisches Wörterbuch der Philosophie. Bd 7, Schwabe \& Co. AG, Basel 1989 Sp. 1748-1752.

Imai M: Kaizen: der Schlüssel zum Erfolg der Japaner im Wettbewerb, 10. Auflage; Wirtschaftsverlag Langen Müller/Herbig, München 1993

Jäckel WH: Definitionen und Typen von Qualitätsindikatoren. in: Programm für Nationale VersorgungsLeitlinien von BÄK, KBV und AWMF: Qualitätsindikatoren - Manual für Autoren-, (Schriftenreihe Band 36); hrsg. v. Zentralstelle der Deutschen Ärzteschaft zur Qualitätssicherung in der Medizin, , Berlin 2009 verfügbar: http://www.aezq.de/mdb/edocs/pdf/schriftenreihe/schriftenreihe36.pdf (abgerufen: 09.02.2012)

Jamtvedt G, Young JM, Kristoffersen DT, Thomson O'Brian MA, Oxman AD (2003): Audit and feedback: effects on professional practice and health care outcomes. The Cochrane Database Syst Rev.2003; 3:CD000259

Jaster HJ: Qualitätssicherung im Gesundheitswesen. Georg Thieme Verlag, Stuttgart 1997

Klosterhuis H (2008): Aktuelle Strategien der Deutschen Rentenversicherung in der Qualitätssicherung der medizinischen Rehabilitation. Prävention und Rehabilitation 20(4), 184-192

Klosterhuis H (2010): Reha-Qualitätssicherung der Rentenversicherung-eine kritische Bestandsaufnahme. RVaktuell, o. O. 260-268

Klosterhuis H, Baumgarten E, Beckmann U, Erbstößer S., Lindow B, Naumann B, Widera T, Zander J (2010): Ein aktueller Überblick zur Reha-Qualitätssicherung der Rentenversicherung. Rehabilitation $\underline{49}$, 356-367 
Köck CM: Qualitätsmanagement: Definition und Abgrenzung; in: Gesundheitsökonomie, Qualitätsmanagement und Evidence-based Medicine; hrsg. v. Lauterbach KW, Schrappe M u. a., Schattauer GmbH, Stuttgart 2001, 282-290

Köhler D, Goeckenjan G, Rünz J (1998): Evolutionäre Qualitätssicherung-Ein neues Konzept zur Verbesserung der Prozess- und Ergebnisqualität. Med Klin 93(3), 191-196

Kopp IB, Selbmann HK, Koller M (2007): Konsensusfindung in evidenzbasierten Leitlinienvom Mythos zur rationalen Strategie. Zeitschrift für ärztliche Fortbildung und Qualität im Gesundheitswesen (ZaeFQ) 101, 89-95Z

Kutschmann M: Entwicklung und Evaluierung eines Verfahrens zur Überprüfung der Prozessqualität in der Schlafmedizin. Med. Diss. Bonn 2004

Kutschmann M, Raschke F, Fischer J (2002): Verbesserung der Prozessqualität in der Schlafmedizin durch ein Peer-Review-Verfahren. Somnologie $\underline{6}$, 85-92

Lindow B, Baumgarten E, Dietrich F (2007): Peer-Review. hrsg.v. Dt. Rentenversicherung Bund GB Sozialmedizin und Rehabilitation. 2007

verfügbar: http://www.suchthilfe.de/basis/checkliste_pr_psy_sucht_5_2007.pdf (abgerufen: 08.10.2011)

Mainz J (2003): Defining and classifying clinical indicators for quality improvement. Int J Qual Health Care 15(6), 523-530

Mayer et al. (2009) = Mayer G, Fietze I, Fischer J, Penzel T, Riemann D, Rodenbeck A, Sitter H, Teschler H (2009): S3-Leitlinie „Nicht erholsamer Schlaf/Schlafstörungen“. Somnologie 13(1), 1-160

McClellan WM, Hodgin E, Pastan S, McAdams L, Soucie M (2004): A randomized evaluation of two health care quality improvement program (HCQIP) interventions to improve the adequacy of hemodialysis care of ESDR patients: feedback alone versus intensive intervention. J Am Soc Nephrol 15, 754-760

Nüllen H, Noppeney T: Lehrbuch Qualitätsmanagement in der Arztpraxis, 3. Auflage, Deutscher Ärzte-Verlag GmbH, Köln 2007

Ohayon MM (2003): The effects of breathing-related sleep disorders on mood disturbances in de general population. J Clin Psychiatry 64(10), 1195- 2000

Penzel T, Hein H, Rasche K, Weeß HG, Fischer J, Hajak G, Mayer G, Wiater A, Zuley J (2000): Leitfaden für die Akkreditierung von schlafmedizinischen Zentren der Deutschen Gesellschaft für Schlafforschung und Schlafmedizin (DGSM). Somnologie $\underline{4}$, 181-187 
Picchietti D, Winkelman JW (2005): Restless Legs Syndrome, Periodic Limb Movements in Sleep, and Depression. Sleep 28(7), 891-898

Pinter E, Vitt KD: Einführung-Umfassendes Qualitätsmanagement als künftiges Thema der Krankenhäuser. in: Umfassendes Qualitätsmanagement für das Krankenhaus: Perspektiven und Beispiele: hrsg. v. Pinter E, Vitt KD. Pmi-Verlag-Gruppe, Frankfurt am Main 1996, 2-20

Robert Koch-Institut (2005) $=$ Robert Koch-Institut (2005): Schlafstörungen (Reihe Gesundheitsberichterstattung des Bundes, Heft 27), Robert Koch-Institut, Berlin 2005

verfügbar:

http://www.rki.de/cln_116/nn_199850/DE/Content/GBE/Gesundheitsberichterstattun g/GBEDownloads $T /$ schlafstoerung,templateld=raw, property=publicationFile.pdf/schl afstoerung.pdf (abgerufen: 05.03.2012)

Rühle KH, Nilius G (2004): Periodische Beinbewegung (PLMS) und Obstruktion der oberen Atemwege im Schlaf. Differenzialdiagnose und Therapie. Eine Falldarstellung. Somnologie $\underline{8(4)}, 149-150$

Schäfer RD: Ärztliches Qualitätsmanagement als berufsspezifische Aufgabe; in: Gesundheitsökonomie, Qualitätsmanagement und Evidence-based Medicine, hrsg. v. Lauterbach KW, Schrappe M u.a., Schattauer GmbH, Stuttgart 2001, 304-309

Schrappe M: Qualität in der Gesundheitsversorgung; in: Gesundheitsökonomie, Qualitätsmanagement und Evidence-based Medicine, hrsg. v. Lauterbach KW, Schrappe M u. a., Schattauer GmbH, Stuttgart 2001, 263-272

Schubert HJ, Ebner H: Evaluierung/Zertifizierung/Akkreditierung von Qualitätsmanagement-Aktivitäten in Gesundheitseinrichtungen; in: Gesundheitsökonomie, Qualitätsmanagement und Evidence-based Medicine, hrsg. v. Lauterbach KW, Schrappe M u.a., Schattauer $\mathrm{GmbH}$, Stuttgart 2001, 419-425

Selbmann HK: Qualitätssicherung-Definition der Qualitätssicherung und ihre Systematik; in: Gesundheitsökonomie, Qualitätsmanagement und Evidence-based Medicine, hrsg. v. Lauterbach KW, Schrappe M u. a.; Schattauer GmbH, Stuttgart 2001, 273281

Sens B, Fischer B, Bastek A, Eckardt J, Kaczmarek D, Paschen U, Pietsch B, Rath S, Ruprecht T, Thomeczek (2007): Begriffe und Konzepte des Qualitätsmanagements, 3. Aufl. GMS Med Inform Biom Epidemiol 3(1): Doc 5 
SGB V (2011a) = SGB V (Sozialgesetzbuch V), Neunter Abschnitt: Sicherung der Qualität der Leistungserbringung, § 135a Abs. 2 SGB V Verpflichtung zur Qualitätssicherung verfügbar: http://www.sozialgesetzbuch-sgb.de/sgbv/135a.html (abgerufen: 12.02.2011)

SGB V (2011b) = SGB V (Sozialgesetzbuch V), Neunter Abschnitt: Sicherung der Qualität der Leistungserbringung, § 137 SGB V Richtlinien und Beschlüsse zur Qualitätssicherung verfügbar: http://www.sozialgesetzbuch-sgb.de/sgbv/137.html (abgerufen: 12.02.2011)

SGB V (2011c) = SGB V (Sozialgesetzbuch V), Neunter Abschnitt: Sicherung der Qualität der Leistungserbringung, § 137a Umsetzung der Qualitätssicherung und Darstellung der Qualität verfügbar: http://www.sozialgesetzbuch-sgb.de/sgbv/137a.html (abgerufen: 12.02.2011)

Statistisches Bundesamt: Gesundheit. Tiefgegliederte Diagnosedaten der Krankenhauspatientinnen und -patienten. 2008. Erschienen am 23.12.2009. Artikelnummer: 5231301087015. Statistisches Bundesamt, Wiesbaden 2009

Stuck BA, Maurer JT, Schredl M, Weeß HG: Praxis der Schlafmedizin-Schlafstörungen bei Erwachsenen und Kindern (Diagnostik Differentialdiagnostik und Therapie). Springer Medizin Verlag, Heidelberg 2009

Szecsenyi J: Was will Qualitätssicherung?; in: Ärztliche Qualitätszirkel-Leitfaden für den niedergelassenen Arzt, hrsg. v. Bahrs O, Gerlach J, Szecsenyi J; 3. Auflage, Deutscher Ärzte-Verlag, Köln 1996, 43-56

Viethen G: Qualität im Krankenhaus-Grundbegriffe und Modelle des Qualitätsmanagements. Schattauer Verlag, Stuttgart 1995 


\title{
8 Anhang
}

\subsection{Checkliste inklusive Beurteilungsmanual zur Qualitätssiche- rung in Schlaflaboratorien der DGSM 2009/2010}

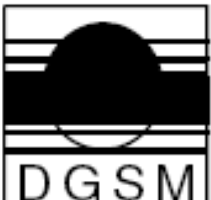

\author{
Deutsche Gesellschaft für \\ Schlafforschung und Schlafmedizin \\ German Sleep Society
}

\section{Checkliste zur Qualitätssicherung der DGSM}

\section{Vorwort}

Die Basis für die Beurteilung stellen die jeweils vorliegenden nationalen und internationalen Empfehlungen und Leitlinien (u. a. der DGSM, AASM, ERS, EFNS) der wissenschaftlichen Fachgesellschaften dar, soweit diese noch aktuell sind. Die Beurteilung richtet sich auch nach den gesundheitspolitischen Bedingungen (z. B. BUB-Richtlinie, DRG Standards, German Appropriatness Evaluation Process GAEP).

\begin{abstract}
Allgemeine Hinweise:
Bitte lesen die Checkliste bzw. die Erläuterungen zu den Einzelpunkten der Checkliste aufmerksam durch. Die Beurteilung erfolgt ab sofort in diesem sowie in den weiteren nun regelmäßig folgenden Durchgängen allein anhand des pseudonymosierten Entlassungsberichts und der mitzuschickenden Nachtprotokolle (Protokoll-Muster siehe Anlage). Vollständige Akten werden nicht mehr begutachtet.

Wir bitten daher alle teilnehmenden Labore, die geforderten Parameter der Checkliste in die Entlassungsberichte zu übernehmen. Lediglich die Polysomnographie-Parameter können als Anlage dem Entlassungsbericht beigefũgt werden, sofern dies dem Standard des Labors entspricht. Diese Parameter müssen aber im Bericht bewertet werden. Für Patienten, die bereits die Stufen 1-3 und ggf. auch Stufe 4 (Diagnostik) der BUB-Richtlinie durchlaufen haben, muss im Entlassungsbericht eine kurze diesbzgl. Stellungsnahme erfolgen.

Bitte haben Sie Verständnis, dass wir nur auf die Angaben im Bericht zurūckgreifen kōnnen und entsprechend keine andere Mōglichkeit haben zu prüfen, ob bestimmte Untersuchungen vor oder während des Klinikaufenthalts durchgefũhrt worden sind bzw. - je nach Fall empfohlen wurden.
\end{abstract}




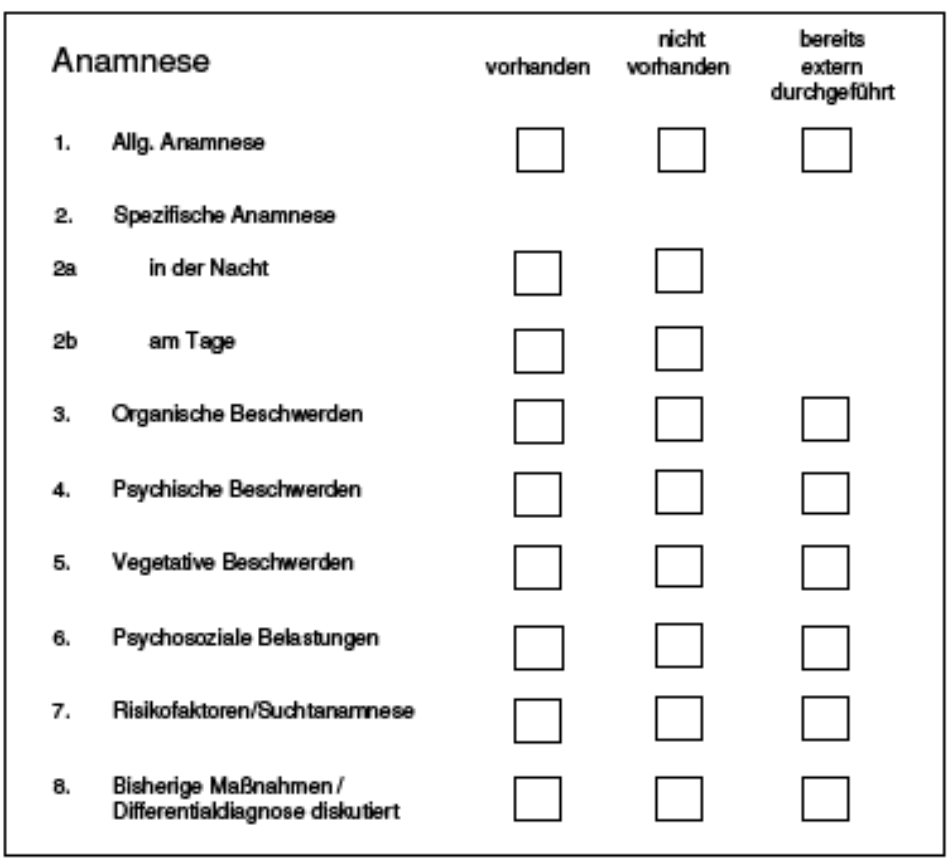

Allgemeine Hinweise zur Beurteilung der im Entlassungsbrief dargestellten Anamnese:

Als svorhanden" wird ein Kriterium dann codiert, wenn hierzu für den jeweiligen Fall ausreichende Angaben im Entlassungsbericht existent sind. Ist der Patient bereits ambulant vordiagnostiziert und wird auf diese Befunde verwiesen, so wird das Kriterium als .extern durchgeführt" codiert. Wird weder auf Vorbefunde verwiesen und finden sich keine eigenen Angaben, so muss „nicht vorhanden" codiert werden. Der Verweis auf Vorbefunde ist auch obligat für Fälle, die nach BUB-Richtlinie bereits die Stufen 1-3 durchlaufen haben, dabei reicht aber ein kurzer entsprechender Satz.

1. Allgemeine

Anamnese: Hier werden Angaben zu Vorerkrankungen und zum Familien-, Sozial-/Berufsstatus erwartet.

2. Spezielle Anamnese / Schlafanamnese, Schlafverhalten: Beschwerden / Störungsverlauf: Hier wird eine chronologische Darstellung des Krankheitsverlaufs der zur Eirweisung führenden Erkrankung oder sonstiger Begleit- oder Folgeerkrankungen der im Vordergrund stehenden Problematik sowie - in Abhängigkeit von spezifischen Fall - eine Aussage zu möglichen auslōsenden Faktoren erwartet (z.B. Tagesmüdigkeit seit $x$ Jahren, etc., siehe auch 9.). Die spezielle Anamnese sollte stets im Entlassungsbericht Erwähnung finden, unabhängig davon, ob bereits ein Vorbefund vorliegt oder nicht; des Weiteren sollte zwischen Müdigkeit und Schläfrigkeit unterschieden werden.

2a. Aktuelle Beschwerden: in der Nacht: Hier sollte die nächtliche Problematik Erwähnung finden (z.B. Atemnot, Rhythmusstörungen, Zähneknirschen, Grübeln, etc.). 2b. Aktuelle Beschwerden: am Tage: Hier sollten die am Tage auftretenden Probleme Erwähnung finden (z.B. Tagesmüdigkeit, Einschlafneigung, etc.). 
3. Organische Beschwerden: Hier wird die Darstellung von Beschwerdesymptomatik, z.B. im Bereich der Herz-Kreislauf-Organe, Atmungsorgane, Schmerzen, etc., erwartet.

4. Psychische Beschwerden: Hier wird die Darstellung von psychischen Beschwerden, z.B. depressive Verstimmung, Wahrnehmungsstōrungen, Verwirrtheit, etc., erwartet.

5. Vegetative Beschwerden: Hier wird die Schilderung von Beschwerden erwartet, die am ehesten als vegetative Beschwerden aufzufassen sind, wie z.B. nächtliches Schwitzen, Himweise auf Hyperventilation, Enuresis, etc.

6. Psychosoziale Belastungen: Hier werden Angaben erwartet über psychische Belastungen (z.B. kritische Lebensereignisse, chronische Konflikte) in Familie und Beruf sowie soziale Belastungen in Familie und/oder Freizeit und/oder mangelnder sozialer Rückhalt.

7. Risikofaktoren/Suchtanamnese: Hier werden Angaben zu Risikofaktoren für die zur Abklärung stehenden Erkrankungen und/oder Folge und Begleiterkrankungen erwartet, wie Adipositas, Fett- und Zuckerstoffwechselstörungen, Nikotin-, Alkoholund Medikamentenabusus etc.

8. Bisherige Maßnahmen/Differentialdiagnose diskutiert: Hier werden Angaben erwartet über bisher im Zusammenhang mit der zur Untersuchung anstehenden Erkrankung oder Grunderkrankung oder hiermit im Zusammenhang stehende Begleitund Folgeerkrankungen, bereits erfolgte diagnostische Maßnahmen, wie z.B. ambulantes nächtliches Monitoring oder sonstige bisher durchgeführte Untersuchungen hinsichtlich der Atmung, des Herz-Kreislauf-Systems, Hals-NasenOhren-ärztliche Untersuchung, neurologische oder psychiatrische Untersuchungen sowie deren Ergebnis. Ebenso werden hier Angaben über bisher erfolgte medikamentöse (Dosierung, evtl. Nebenwirkungen, Compliance) oder nicht medikamentöse (z.B. allgemeine, operative, apparative Maßnahmen) Behandlungen mit Wirkung, Nebenwirkung und Compliance erwartet. 


Diagnostik
9. Subjektive Befindlichkeit
10. Allg. körperlicher, neurologischer,
psychiatrischer Status
11. Größe, Gewicht, BMI
13. Klinisches Labor
14. Kardioresp. Polysomnographie/
15. Kolygraphie oder Monitoring
16. Nomorbide Störung erkannt/
diagnostiziert

Allgemeine Hinweise zur Beurteilung der im Entlassungsbrief dargestellten Diagnostik:

Als „Vorhanden“ wird ein Kriterium dann codiert, wenn hierzu für den jeweiligen Fall ausreichende Angaben im Entlassungsbericht existent sind, bei dem Nichtzutreffen eines Kriteriums für den spezifisch zu beurteilenden Fall wird "entfällt" codiert. Ist der Patient bereits ambulant vordiagnostiziert und wird auf diese Befunde (je nach Art des Befundes in der Regel nicht älter 6 Monate, Laborbefunde in der Regel nicht älter als 4 Wochen, HLATypisierung ohne Zeitbegrenzung) verwiesen, so wird das Kriterium als „extern durchgeführt“ codiert. Wird weder auf Vorbefunde verwiesen und lassen sich keine eigenen Angaben finden, so muss "nicht vorhanden" codiert werden. Der Verweis im Bericht auf Vorbefunde ist auch obligat für Fälle, die nach BUB-Richtlinie bereits die Stufen 1-3 durchlaufen haben, dabei reicht aber ein kurzer entsprechender Satz.

Wird eine weiterführende Diagnostik empfohlen, ggf. auch erst nach Abschluss aller anderen Untersuchungen einschl. einer Polysomnographie im der Epikrise/Zusammenfassung des Entlassungsberichts, so ist „Empfehlung ist erfolgt“ zu codieren. Das Item „Empfehlung fehlt“ ist spezifischen diagnostischen Prozessen vorbehalten, bei denen nach Abschluss aller anderen Untersuchungen ein entsprechende weiterführende/weiter ausschließende Diagnostik zu erwarten gewesen wäre. Nur wenn sich eine schlafbezogenen Atmungsstörung erst während der Polysomnographie herausstellt (d. h. ohne wegweisende anamnestischen Hinweise oder bei negativem Polygraphie Befund), kann eine entsprechende Empfehlung ausgesprochen werden. In allen anderen Fällen sollten zumindest Lungen- und Nasenfunktion im Vorfeld erfolgt sein oder während des Aufenthalts erfolgen. Entsprechendes gilt für das klinische Labor bei RLS/PLMD-Fällen bei denen sich eine entsprechende Pathologie erst nach der Polysomnographie herausstellt sowie analog für andere Erkrankungen.

9. Subjektive Befindlichkeit: Hier wird der Einsatz standardisierter Fragebögen, Schlaftagebüchern etc zum Schlaf-Wach-Verhalten sowie zur Schläfrigkeit mit entsprechender Beurteilung erwartet. Mögliche Fragebögen wären z.B. Pittsburgher Schlafqualitäts-Index (PSQI), Allgemeiner Schlaffragebogen (ASF), SymptomBewertungsbogen (SBB), Stanford Sleepiness Scale (SSS), Epworth Sleepiness Scale (ESS), RLS-Severity-Scale, Schlafverhaltensfragebogen nach Siegrist, etc. 
10. Allgemeine klinische Untersuchung: Bei diesem Merkmal werden Angaben erwartet über die medizinisch übliche Allgemeinuntersuchung (Ganzkörperstatus) einschl. eines neurologischen und psychiatrischen Status.

11. Größe, Gewicht, BMI: Hier sollten die entsprechenden Parameter aufgeführt sein

12. Klinisches Labor: Hier wird eine Aussage der üblichen internistischen RoutineLaborparameter unter besonderer Berücksichtigung des spezifischen Falls (z. B. kardiovaskuläre Risikofaktoren bei OSAS, Ferritin, Kreatinin bei RLS/PLMD) erwartet. Bei SBAS kann das Labor entfallen, sofern es in den Voruntersuchungen ohne wesentliche weiterführende Auffälligkeiten war.

13. Leistungs- und Vigilanzuntersuchung: Hier werden Aussagen zu z.B. polygraphischen Vigilanzuntersuchungen (MSLT/MWTs) und/oder psychologische Testverfahren in Abhängigkeit vom Fall erwartet (z.B. Quatember Maly, d2-Test, Fahrsimulator, Pupillometrie, VIGIMAR, PVT etc.).

14. kardiorespiratorische Polysomnographie / Polygraphie oder Monitoring: Hierunter soll angegeben werden, ob bereits entsprechende Untersuchungen im Vorfeld durchgeführt worden sind.

15. komorbide Störungen erkannt/diagnostiziert: Hier soll bewertet werden, ob komorbide Störungen anhand der Anamnese und ggf. aufgrund im Labor durchgeführter Untersuchungen erkannt worden sind (z. B. Dysgnathie, verminderte Nasendurchgängigkeit, Depression, etc.). Ist eine komorbide Störung erkannt/diagnostiziert worden, so wird dies als „vorhanden“ kodiert, ist sie aus den aufgeführten Befunden ersichtlich, wird aber weder in den Diagnosen noch in der Epikrise aufgeführt, so wird „nicht vorhanden“ codiert. Entsprechendes gilt für ausgesprochene/nicht ausgesprochene Empfehlungen zur weiteren Diagnostik.

16. notwendige Zusatzuntersuchungen: Hier wird bewertet, ob zusätzliche Untersuchungen durchgeführt worden sind (,vorhanden“), oder erwartet und nicht durchgeführt wurden („nicht vorhanden“). Gleiches gilt für die entsprechenden Empfehlungen. 


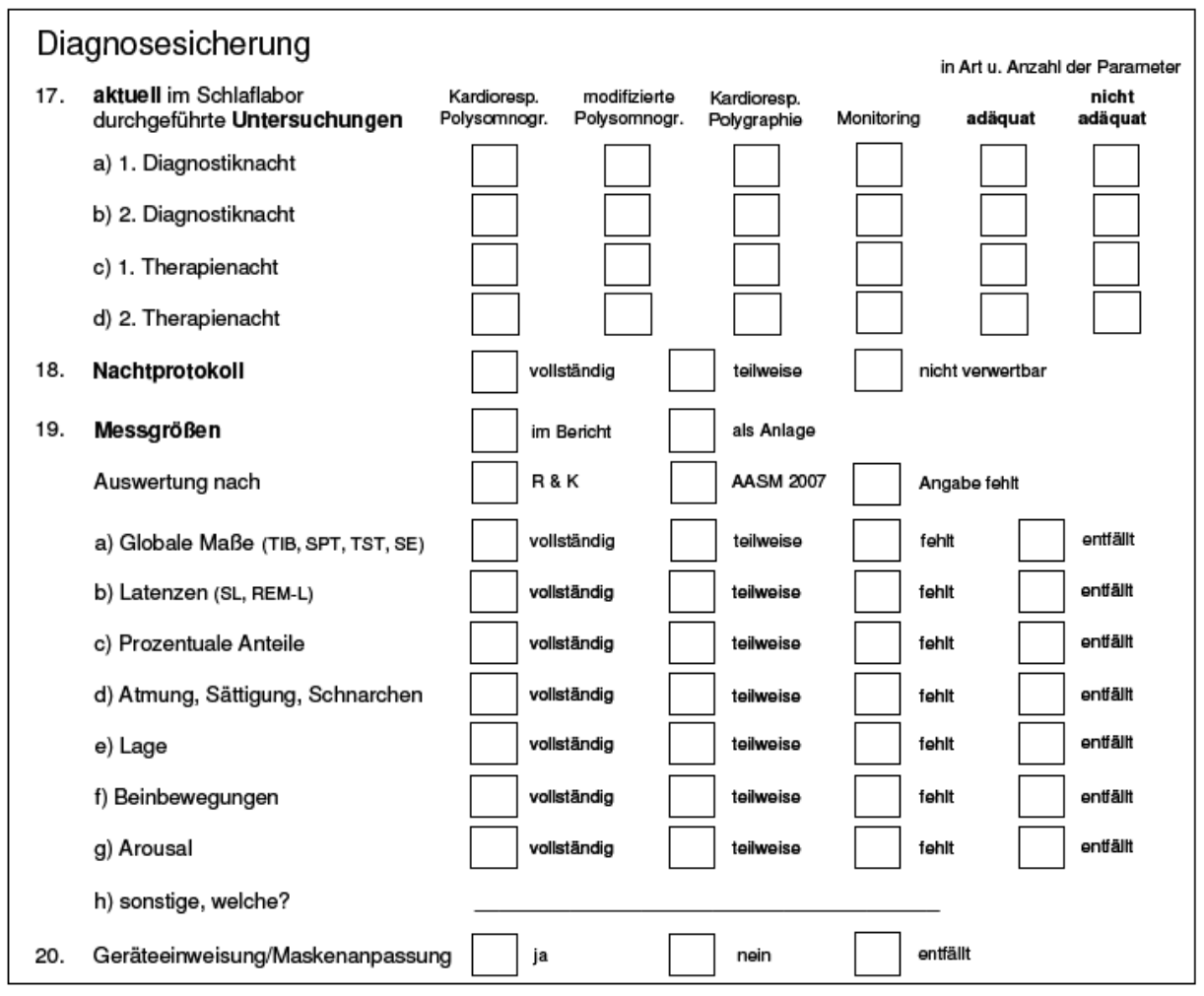

Allgemeine Hinweise zur Beurteilung der Diagnosesicherung:

Als „Vollständig“ wird eine Messgröße dann codiert, wenn hierzu für den jeweiligen Fall die Angaben im Entlassungsbericht (ggf. als Anlage) als Wert vorhanden sind, bei dem Nichtzutreffen eines Kriteriums für den spezifisch zu beurteilenden Fall wird „entfällt“ codiert. Liegt nur ein Teil der Werte der entsprechenden Gruppe vor, so ist „teilweise" zu werten. Gleiches gilt analog für das Nachtprotokoll (siehe 21), dass ebenso wie die GanzNacht-Übersicht (Schlafprofil samt weiterer erfasster Parameter) und im Falle einer kardiorespiratorischen Polysomnographie auch ein Bespielausdruck dem Entlassungsbericht beiliegen muss. Es muss erkennbar sein, dass eine manuelle Korrektur/Durchsicht sowohl der Schlafstadienanalyse als auch der weiteren Parameter erfolgt ist. Sofern dies nicht automatisch vom Gerät erfolgt, muss dies z. B. per Unterschrift des Auswerters erfolgen.

17. Aktuell durchgeführte Untersuchungsnächte: Hier wird codiert welche Untersuchung in den Diagnostik- und ggf. Therapienächten durchgeführt wurde. Zusätzlich wird beurteilt, ob die gewählte Untersuchung in spezifischen Fall nach Art und Anzahl der abgeleiteten Parameter adäquat war.

- Kardiorespiratorische Polysomnographie: Schlafableitung inkl. respiratorischer Messgrößen, EKG und peripherem EMG der Tibialis ggf. plus weiteren Parametern je nach Fall

- modifizierte Polysomnographie: Schlafableitung und EKG sowie mit - je nach spezifischem Fall - Aufzeichnung weiterer Parameter wie Atmungsparameter oder 
peripheres EMGs der Tibialis, der Masseter etc., weiterer EEGs etc., jedoch keine vollständige kardiorespiratorische Polysomnographie

- Kardiorespiratorische Polygraphie: Untersuchung respiratorischer Messgrößen, EKG u.a., $\quad$ mindestens 6 Kanäle nach BUB-Richtlinie - Nächtliches Monitoring: Registrierung von 4 Kanälen oder weniger in der Untersuchung.

HINWEIS: eine kardiorespiratorische Polysomnographie kann nur dann als solche gewertet werden, wenn mindestens ein Beispielausdruck beiliegt, aus dem hervorgeht, welche Kanäle erfasst worden sind.

18. Protokoll der Nacht: Standardisiertes Protokoll über Patientenverhalten und/oder vorgenommene Zeitpunkte der Therapieeinstellung (Druckwerte) einschl. protokollierter Biosignal-Eichung, Impedanzmessung, Licht an/aus-Zeiten usw. Ebenso muss die Anwesenheit und die Qualifikation der Nachtwache und des ärztlichen Hintergrunds dokumentiert sein. Sind alle Angaben komplett wird „vollständig“, beim Fehlen einiger Parameter „teilweise“ codiert. Fehlen dem Protokoll alle o. g. wichtigen Angaben so ist ein "nicht verwertbar" zu vergeben. Ausnahme: bei Ableitungen bei Kindern entfällt die Biosignal-Eichung.

19. Messgrößen: Diese Frage bezieht sich vor allem darauf, ob die Ergebnisse der Untersuchungen zahlenmäßig im Entlassungsbericht (bzw. als Anlage zum Bericht) erscheinen.

- Auswertung nach: Hier muss ab dem 01.01.2009 It. Beschluss der DGSMMitgliederversammlung im Oktober 2008 in Kassel im Entlassungsbericht angegeben werden, ob die Auswertung nach den Kriterien von R \& K (1968, 2 EEGs zentral) oder nach den Regeln der AASM (2007, 3 EEGs: okzipital, zentral und frontal) erfolgt.

a) globale Schlafmaße (time in bed (TIB), sleep period time (SPT), total sleep time (TST), Schlafeffizienz (SE))

b) Schlaflatenzen (Einschlaflatenz bis zum Stadium 2 bzw. N2 (SL), REM-Latenz)

c) prozentuale Schlafstadienanteile für alle Schlafstadien

d) Atmung (Apnoe-Index, Hypopnoe-Index, Apnoe-/Hypopnoe-Index, Verteilung nach Stadien und /oder Lage etc.),

Sauerstoffsättigung (SaO2) (Anzahl, Häufigkeit, Ausmaß von Entsättigungen), Schnarchen (Häufigkeit, Schnarchindex, Intensität etc.)

e) Körperposition (Länge und Dauer in der jeweiligen Position, bei Patienten mit schlafbezogenen Atmungsstörungen mindestens aber eine Aussage, ob die entsprechenden Ereignisse Lagebezogen sind)

f) Beinbewegungen (Index periodischer Beinbewegungen insgesamt, Index periodischer Beinbewegungen mit Arousal, Verteilung im Nachtverlauf). Bei periodischen Beinbewegungen, die nicht im Index erfasst werden (z. B. in der Einschlafphase oder am Ende eine Apnoe/Hypopnoe) wird zumindest eine entsprechende Aussage im Bericht erwartet. Analog gilt dieses Item auch für abnorme Verhaltensweisen in der Nacht ( $z$. B. Parasomnien)

g) Arousals (zahlenmäßige Angaben über Summe oder Index von spezifizierten Arousals, mindestens aber eine Aussage darüber, ob bei Patienten mit a) periodischen Beinbewegungen oder schlafbezogenen Atmungsstörungen diese Ereignisse zu Arousals führen, b) Insomnie ohne organisches Korrelat reine EEG-Arousal vorhanden sind)

h) Sonstige (z.B. Oesophagusdruck, Blutdruck, $\mathrm{CO} 2$, etc.)

20. Geräteeinweisung/Maskenanpassung: Erfolgte eine dokumentierte Geräteeinweisung und sorgfältige Maskenanpassung, so gilt sie als durchgeführt. 


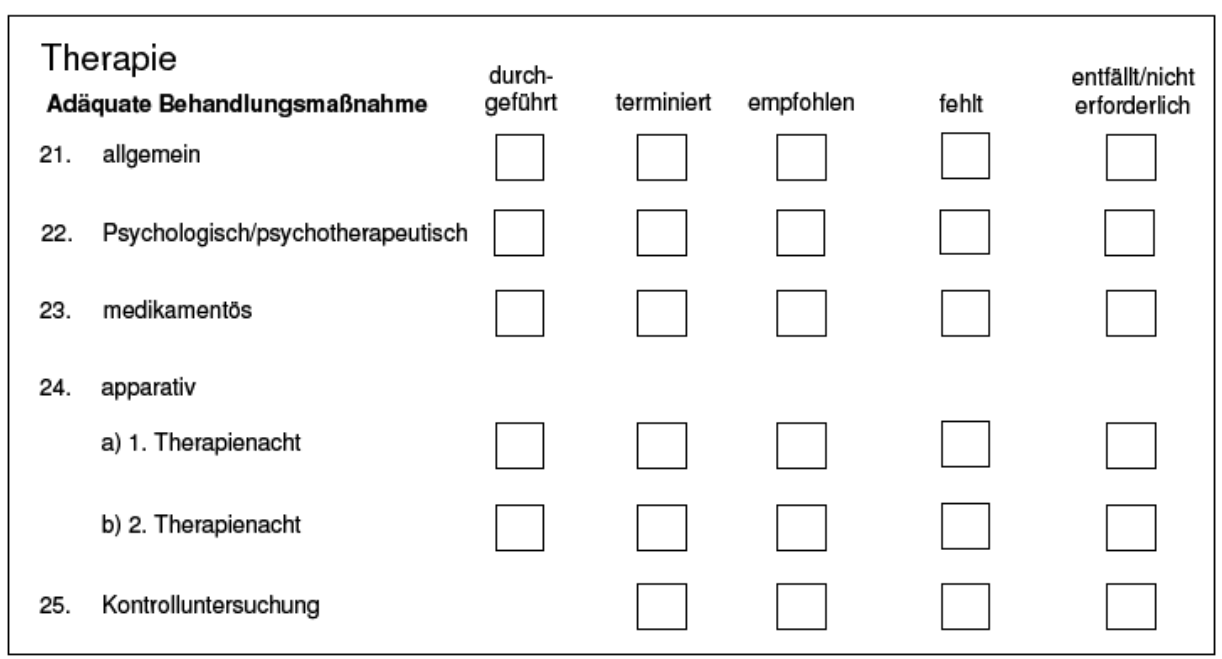

Allgemeine Hinweise zur Beurteilung der Therapie:

Als ,durchgeführt" wird ein Item dann codiert, wenn dies bereits während des Schlaflaboraufenthalts erfolgt ist, als "terminiert" wenn hierfür ein fester Termin (ggf. auch Wartelistenplatz) festgelegt wurde. „Empfohlen“ wird verwendet sofern ein entsprechender Vermerk im Entlassungsbericht vorhanden ist. Das Kriterium "Fehlt" beinhaltet dagegen, dass eine entsprechende Maßnahme bzw. die Empfehlung hierzu nach Lage des spezifischen Falls therapeutisch sinnvoll gewesen wäre, aber nicht vorhanden ist.

21. Allgemeine Behandlungsmaßnahme: Hier wird erwartet, dass bezogen auf das schlafmedizinische Krankheitsproblem allgemeine Behandlungsmaßnahmen als Therapie bereits während des Aufenthaltes eingeleitet (Codierung als durchgeführt) oder empfohlen wurden, wie z.B. Gewichtsreduktion, Schlafhygiene, Vermeidung Rückenlage etc.

22. Psychologische/Psychotherapeutische Behandlungsmaßnahmen: Hier wird sie als durchgeführt codiert, wenn sie direkt in der Klinik begonnen wird, z. B. Stimulus-KontrollTherapie, Entspannungsverfahren, Schlafrestriktion, kognitive Umstrukturierung, Psychotherapie, Verhaltenstherapie, Ernährungsberatung

23. Medikamentöse Behandlungsmaßnahmen: Hier wird erwartet, dass die eingeleitete medikamentöse Therapie bereits in ihrer Auswirkung auf das schlafmedizinische Problem validiert wurde (Codierung als durchgeführt). Wurde sie nur verordnet, so ist dies mit empfohlen zu codieren.

24. Apparative Behandlungsmaßnahmen: Hier wird erwartet, dass die erforderlichen Therapienächte nach Diagnosestellung bzw. differentialdiagnostischer Abklärung eingeleitet, terminiert oder empfohlen wurden. Die 2.Therapienacht soll direkt nach der 1. Therapienacht erfolgen (Codierung als durchgeführt). Es muss vermerkt in Entlassungsbericht oder im Nachtprotokoll vermerkt sein, mit welchem Beatmungsmodus, Gerät, Druck und Zusatz (WLB) der Patient versorgt wurde und wer der Ansprechpartner bei Problemen ist.

25. Kontrolluntersuchung: Hier wird eine Angabe erwartet, ob eine polygraphische oder polysomnographische Kontrolluntersuchung terminiert oder empfohlen wurde. 


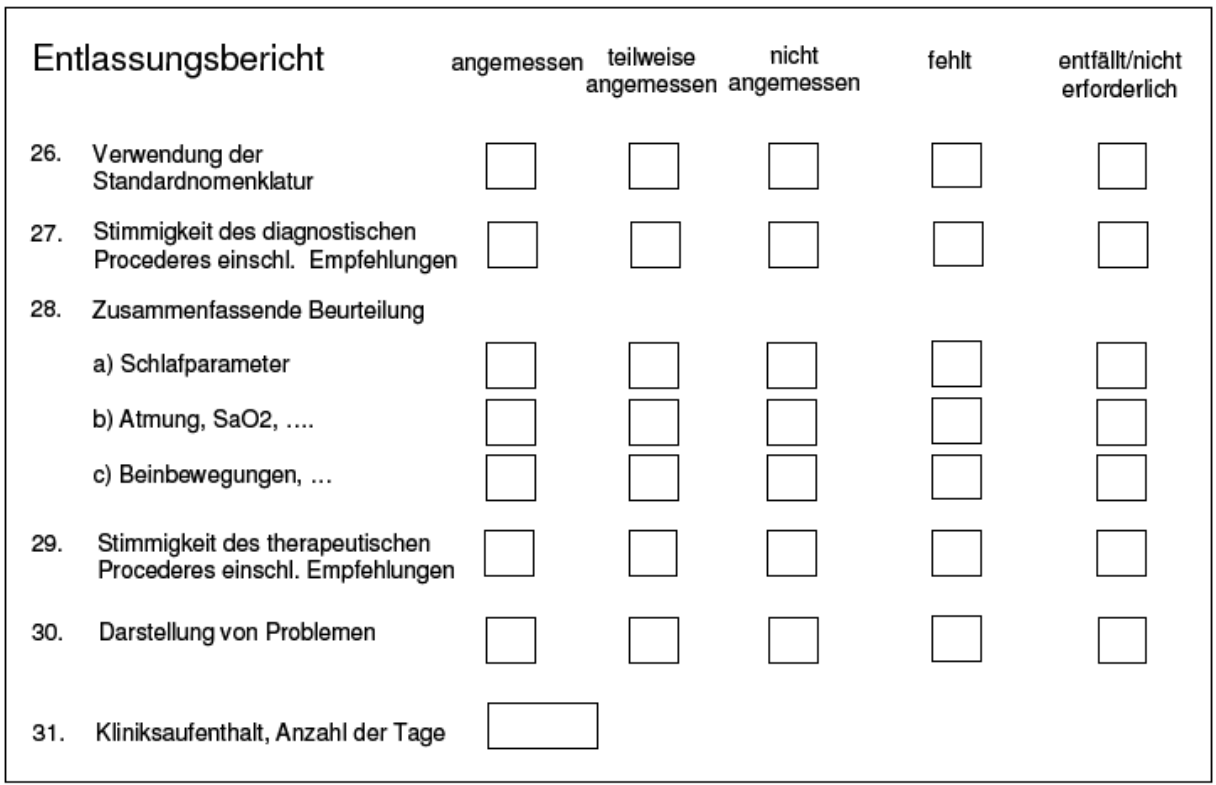

Allgemeine Hinweise zur Beurteilung des Entlassungsberichts:

Der Begriff „angemessen“ wird hier je nach spezifischem Fall durch die jeweiligen Gutachter spezifiziert. Das Kriterium „fehlt“ beinhaltet, dass eine Beurteilung der erfassten Parameter im Entlassungsbericht fehlt.

26. Verwendung der Standardnomenklatur: Hier soll beurteilt werden, ob die Standardnomenklatur bzgl. Diagnosen (ICSD-2 und ICD-10) und Polysomnographie/ PolygraphieParametern eingehalten wurde.

27. Stimmigkeit des diagnostischen Procederes einschl. ggf. erfolgter Empfehlungen: Die durchgeführten diagnostischen Maßnahmen und Empfehlungen müssen die bisherigen bekannten/vorliegenden Befunde sinnvoll berücksichtigen und darauf aufbauen. (Welche weiteren diagnostischen Maßnahmen im Einzelnen durchgeführt/empfohlen wurden bzw. deren Empfehlung erwartet worden wäre, wird unter der Rubrik Diagnostik codiert.). Ebenso ist hierbei zu berücksichtigen, ob die Diagnose mit den erhobenen dargestellten Befunden übereinstimmt und eine ggf. erfolgte Empfehlung zur weiteren differentialdiagnostischen Abklärung mit den erhobenen Befunden begründet wird.

28. Zusammenfassende Beurteilung: Hier soll codiert werden, ob eine angemessene Beurteilung von Schlaf-, Atmungsparametern und Beinbewegungen usw. im Entlassungsbericht gegeben wurde.

29. Stimmigkeit des therapeutischen Procederes einschl. Empfehlungen: Hier wird codiert, ob die erfolgte Therapie bzw. die Empfehlung hierzu sich schlüssig und sinnvoll aus den erhobenen Befunden ergibt und eine Therapiebegründung im Entlassungsbrief begründet wurde.

30. Problemdarlegung: z.B. Komplikationen, First Night Effekt, Compliance, technischer Art, Maskenanpassung, Nebenwirkungen etc.

31. Kliniksaufenthalt: Hier soll die Anzahl der Tage (Aufnahme- und Entlassungstag gelten als ein Tag) des Gesamtaufenthaltes in der Klinik angegeben werden. 


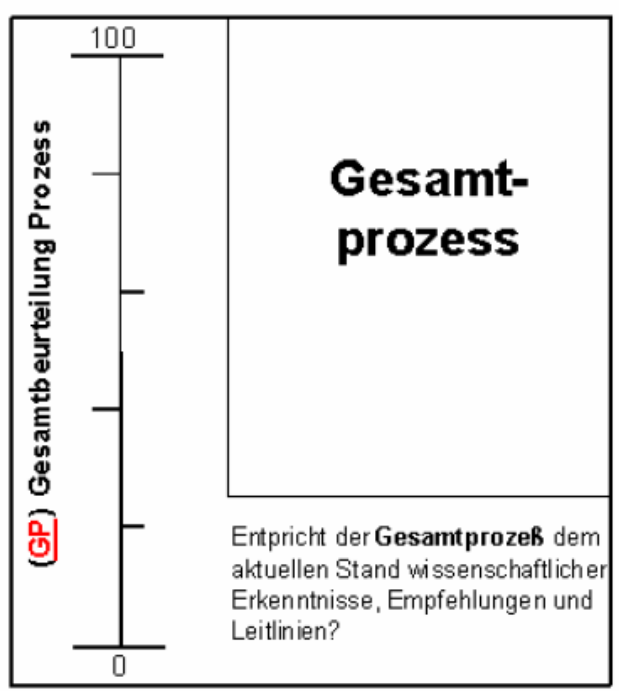

Bitte beurteilen Sie mittels Analogskala, ob der Gesamtprozess dem aktuellen Stand wissenschaftlicher Erkenntnisse, Empfehlungen und Leitlinien entspricht: " 0 " bedeutet "überhaupt nicht", "100" bedeutet "vollständig".

\section{Revisitation des Labors}

empfohlen

nicht erforderlich

Anmerkungen (werden anonym an das beurteilte Labor weitergegeben): 


\subsection{Deckblatt - Allgemeine Angaben zum Schlaflabor}

\section{Qualitätssicherung Prozessqualität der DGSM}

Deckblatt - Allgemeine Angaben zum Schlaflabor -

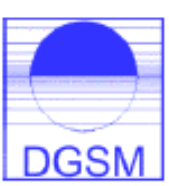

1a) Adresse des Labors: Name:

zur Verwendung

Abtl:

auf der Homepage

Klinik:

Str.:

PLZ, Ort:

Telefon:

Fax:

Email:

Homepage:

1b) Verantwortlichkeiten :

Leiter/in der Klinik/Abtl. :

Leiter/in des Schlaflabors :

Medizinisch Verantwortliche/er:

1c) veränderte Räumlichkeiten seit letzter Reevaluation:

ja $\square \quad$ nein $\square$

wenn ja, bitte hier genaue Angabe (Umzug, Bettenerweiterung, ...), ggf. Raumplan beifügen, sowie Ausstattung der Räume (Ableittechnik, Verdunklung, Audio-Video-Überwachung etc.)

1d) Anzahl der Ableitplätze

Polysomnographiebetten Polygraphiebetten Monitoringbetten

1e) Ambulanz $\quad$ ja $\square \quad$ nein $\square$ 


\section{2) Geräte}

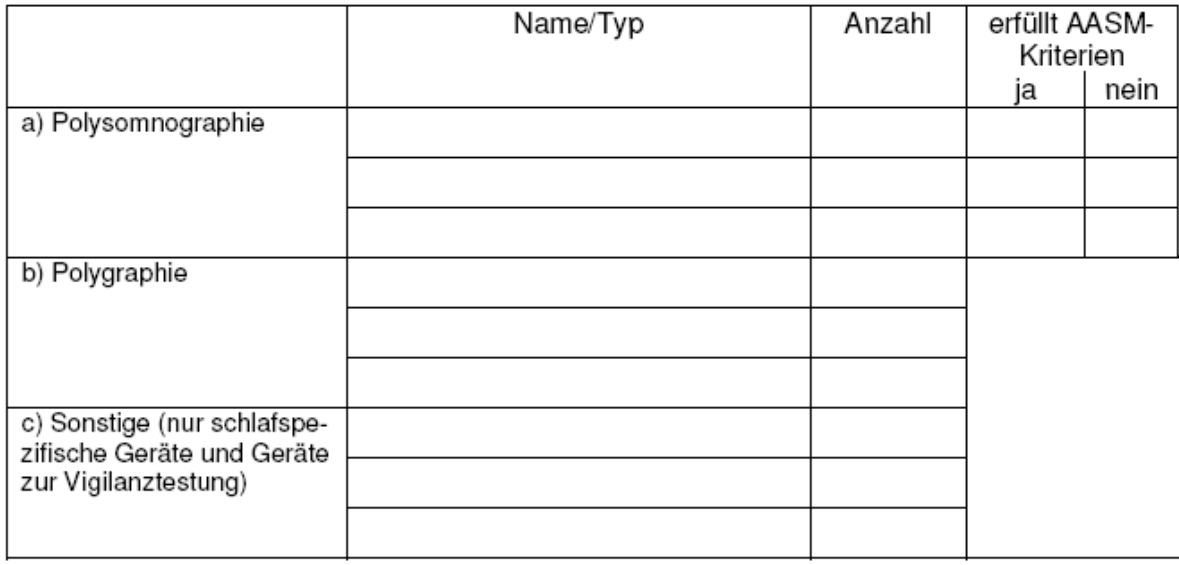

3) Angaben zu durchgeführten Messungen im letzten Kalenderjahr, bitte Auszug des aktuellsten Qualitätsberichts der Abtl./Klinik mit Anzahl schlafrelevanter Diagnosen beilegen

\begin{tabular}{|l|l|l|l|}
\hline & Polysomnographien & Polygraphien & MSLTs / MWTs \\
\hline Anzahl & & & \\
\hline
\end{tabular}

4) Mitarbeiter und Qualifikation (bitte die Mitarbeiter des letzten Kalenderjahres namentlich auffären)

\begin{tabular}{|c|c|c|c|c|c|c|c|c|c|c|c|c|}
\hline \multirow{2}{*}{$\begin{array}{l}\text { Funktion u. Qualifikation } \\
\text { (z. B. Arzt, FA for..., } \\
\text { Psychologe, Krankenpfleger, } \\
\text { MTA, MDA, sitzwache, } \\
\text { Schreibkraft, Techniker, ...) }\end{array}$} & \multirow[t]{2}{*}{ Name } & \multirow{2}{*}{$\begin{array}{l}\text { Std. pro } \\
\text { Woche im } \\
\text { Schlaflabor, } \\
\text {-ambulanz } \\
\text { tätig }\end{array}$} & \multicolumn{2}{|c|}{$\begin{array}{l}\text { DGSM- } \\
\text { Mitglied }\end{array}$} & \multicolumn{2}{|c|}{$\begin{array}{l}\text { Qualifikations- } \\
\text { nachweis } \\
\text { Somnologie }\end{array}$} & \multicolumn{2}{|c|}{$\begin{array}{c}\text { Zusatz- } \\
\text { bezeichnung } \\
\text { Schlafmedizin }\end{array}$} & \multicolumn{2}{|c|}{$\begin{array}{l}\text { KV-Zulassung, } \\
\text { Polikininik, etc nur for } \\
\text { Schlatumedizinische } \\
\text { Belange }\end{array}$} & \multicolumn{2}{|c|}{$\begin{array}{l}\text { Weiterbildungs- } \\
\text { ermächtigung } \\
\text { Schlafmedizin }\end{array}$} \\
\hline & & & ja & nein & ja & nein & $\mathrm{ja}^{*}$ & nein & ja & nein & $\begin{array}{c}\mathrm{Ja} \\
\text { (Monate) }\end{array}$ & nein \\
\hline \multicolumn{13}{|l|}{ Klinik-/ Abtl.-Leiter/in ${ }^{(1)}$} \\
\hline \multicolumn{13}{|l|}{ Laborleiter/in $^{(1)}$} \\
\hline \multicolumn{13}{|l|}{ Medizinische/r Leiter/in } \\
\hline & & & & & & & & & & & & \\
\hline & & & & & & & & & & & & \\
\hline & & & & & & & & & & & & \\
\hline & & & & & & & & & & & & \\
\hline & & & & & & & & & & & & \\
\hline & & & & & & & & & & & & \\
\hline & & & & & & & & & & & & \\
\hline & & & & & & & & & & & & \\
\hline & & & & & & & & & & & & \\
\hline & & & & & & & & & & & & \\
\hline & & & & & & & & & & & & \\
\hline & & & & & & & & & & & & \\
\hline & & & & & & & & & & & & \\
\hline & & & & & & & & & & & & \\
\hline & & & & & & & & & & & & \\
\hline & & & & & & & & & & & & \\
\hline
\end{tabular}

(1) Leiter/in der Klinik/Abtl. und/oder Laborleiter/in muss Mitglied der DGSM sein und zusătzlich über den Qualifikationsnachweis Somnologie (DGSM) und/oder die Zusatzbezeichnung Schlafmedizin der Ärztekammer verfügen.

* Bitte Kopie der Urkunde beifágen - sofern noch nicht mit der Anmeldung abermittelt. 


\title{
8.3 Merkblatt zum Qualitätssicherungsverfahren der DGSM
}

\author{
Merkblatt \\ Qualitätssicherung der DGSM
}

Für jeden ausgewählten Patienten müssen folgende Unterlagen eingereicht werden:

- Protokolle aller zum aktuellen Aufenthalt gehörigen Nächte

- Entlassungsbericht ggf. mit gesondertem Auswertungsbericht

- Alle Unterlagen müssen

- pseudonymisiert sein. Das heißt Namen und Vornamen des Patienten schwärzen und mit Namenskürzel (je 1. Buchstabe Vornamen, Nachnamen) versehen, das Geburtsjahr sollte bleiben (um ganz sicher zu gehen, dass der Name nicht lesbar ist erst kopieren dann Kopie schwärzen und dann davon eine Kopie versenden)

- mit Namen und Ort der Klinik/des Schlaflabors/der Praxis versehen sein.

Die Protokolle sollen neben Namenskürzel mindestens folgende Angaben enthalten

- Name und Unterschrift der Sitzwache

- Name und Unterschrift des überwachenden Arztes

- Uhrzeiten (oder Epochenangabe) der Kalibrierung, der Impedanzmessung, der BiosignalEichung (Biosignal-Eichung enttällt bei Kindern)

- Uhrzeiten von Licht aus und endgültigem Licht an

- Uhrzeit des Beginns der Aufzeichnung

- Mögliche Probleme

- Auflistung von Druckeinstellung, -veränderung, Medikamenten, Toilettengänge, zwischenzeitliches Licht an etc (mit Uhrzeit oder Epochen-Nr.)

- Auflistung technischer Veränderungen (neue Verkabelung, Nachkleben, Veränderung der Empfindlichkeiten etc) mit Uhrzeit oder Epochen-Nr.

- Auffallende Ereignisse wie Parasomnien, auffälliges RLS, lautes Schnarchen etc. mit Uhrzeit oder Epochenangabe

Aus dem Entlassungsbericht soll hervorgehen:

- die Anamnese einschl. möglicherweise bereits vorhandener Vorbefunde samt deren Bewertung

- die im Vorfeld oder aktuell durchgeführte Diagnostik samt Bewertung der Befunde

- die Medikation

- alle schlafmedizinisch relevanten Diagnosen

- die Anzahl der aktuellen Nächte samt deren Bewertung hinsichtlich der gemessenen Parameter

- die Therapie bzw. Therapieempfehlung einschl. deren schlüssigen Ableitung aus den erhobenen/bekannten Befunden

- ggf. eine weiterführende Diagnostik mit schlüssiger Ableitung aus den Befunden und einer kurzen Diskussion möglicher Differentialdiagnosen

Entweder im Protokoll oder im Auswertungs-Bericht (sofern er mitgeschickt wird) oder im Entlassungsbericht müssen folgende Angaben ersichtlich sein:

- Welche Ableitung mit welchen Parametern wurde gemacht (zum Nachweis einer kardioresp. Polysomnographie mit mind. R\&K-Parametern, EKG, Atmung, PLMs bitte mindestens einen Ausdruck einer Epoche mitschicken, aus dem dies eindeutig hervorgeht)

- Wie wurde ausgewertet (R\&K oder AASM)

- Wurde manuell korrigiert (zum Nachweis bitte Hypnogramm, Trend o.Ä. mit senden, bei dem sowohl die automatische als auch die manuelle Analyse dargestellt ist. Sofern die Auswertung nicht typischerweise als Anlage dem Entlassungsbericht beigefügt wird, muss dieser Ausdruck Name und Unterschrift des Auswerters und des Befunderstellers)

- Die in der Checkliste geforderten Angaben bzgl. Schlaf, Atmung, usw. müssen entweder im Entlassungsbericht ausgeführt werden oder als Anlage des Berichts beigefügt sein.

- Es muss ersichtlich sein, ob es sich um eine Diagnostik, Neueinstellung, Kontrolle etc. handelt.

- Sofern zutreffend muss die Angabe auf welches Gerät (Typ und Hersteller) und Druck der Pat. eingestellt wurde, vorhanden sein 


\subsection{Muster Polysomnographie - Protokoll}

\section{Polysomnographie-Protokoll}

1. Patient (Initialen):

Besonderheiten (einschl. Medikation):

\section{Vor der Aufzeichnung}

Datum der Untersuchung:

Name Ableiter:

Name verantw. Arzt:

Ableitprogramm (Konfiguration): Ausdruck der Konfiguration oder Beispielseite beilegen

Zusatzkanäle:

Typ der Untersuchung: $\square$ Diagnostik $\square$ Neueinstellung $\square$ Kontrolle $\quad \square$ Umstellung

Bei nächtl. Überdrucktherapie: Gerät (Typ/Hersteller):

Modus: $\square$ CPAP $\square$ Bi-Level $\square$ APAP $\square$ anderer:

Anfangsdruck: bzw. Druckgrenzen:

Weitere Parameter (Anfangseinstellungen):

Warmluft-Befeuchter

Maske: (Art, Typ, Größe):

\section{Durchführung}

Beginn der Aufzeichnung: Uhr

Technische Kalibrierung (Eichzacken) durchgeführt

Impedanzmessung: Alle Impedanzen unter

Biosignal-Eichung: Alle Kanäle artefaktfrei?

Nicht korrigierbare Artefakte / Abweichungen:

Licht aus: Uhr

Beobachtungen während der Nacht (s. Notizenliste):

Licht an: Uhr

Letzte Geräte-Einstellung (End-Druck, weitere Parameter):

Besonderheiten der Ableitung, Bemerkungen:

\section{Auswertung}

Name Auswerter:

Auswertung Schlafstadien: nach $\square$ R\&K $\square$ nach AASM $\square$ keine visuelle Auswertung

Auswertung kardiorespiratorische Parameter:

- manuelle Auswertung / Korrektur der Atemereignisse: $\square$ ja $\square$ nein

- manuelle Auswertung/ Korrektur der Beinbewegungen: $\square$ ja $\square$ nein 


\section{Ergebnis der Auswertung}

Globale Schlafmaße: TIB

SPT

TST

SE (\% von TIB).

Latenzen: Schlaflatenz (ab Licht aus):

REM-Latenz (ab Schlafbeginn):

Prozentuale Anteile:

\begin{tabular}{|l|l|l|l|l|l|l|l|}
\hline Bezug & Wach & S1/N1 & S2 / N2 & S3 / N3 & S4 / -- & REM / R & MT / -- \\
\hline & & & & & & & \\
\hline
\end{tabular}

Arousals:

Anzahl Gesamt:

Respirator.:

PLM-Arousals:

Arousal-Index:

Respiratorische Parameter

Apnoen

obstruktiv: Anzahl:

max. Dauer:

zentral: Anzahl: max. Dauer:

gemischt: Anzahl: max. Dauer:

Hypopnoen (Definition:

Anzahl: max Dauer:

Apnoe-Hypopnoe-Index (/Std. Schlaf):

Sauerstoffsättigung: basale:

Anzahl Entsättigungen: mittlere: tiefste:

\section{Beinbewegungen}

PLM-Index: PLM-Arousal-Index:

\section{Bemerkungen:}

\section{Unterschrift Auswerter}

\section{Befundung}

Befundung durch:

Befundbericht anhand der Originaldaten erstellt $\quad \square$ ja $\quad \square$ nein

Befund und Beurteilung:

Unterschrift des Befunderstellers

(Arzt / Psychologe) 


\subsection{Erläuterungen zum Polysomnographie - Protokoll}

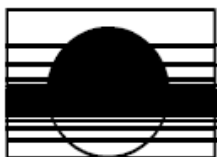

\section{Deutsche Gesellschaft für Schlafforschung und Schlafmedizin \\ German Sleep Society}

D GSM

\section{Erläuterungen zum Polysomnographie-Protokoll}

Das Polysomnographie-Protokoll stellt keine Vorlage für die Verwendung in einem Schlaflabor dar.

Die genannten Angaben sind für die Beurteilung im Rahmen der Qualitätssicherung erforderlich. Um die Begutachtung zu vereinfachen, sollen die entsprechenden Daten in diesem Protokoll eingetragen werden, wenn sie nicht ohne weiteres aus dem Arztbrief oder anderen beigefügten Anlagen ersichtlich sind.

Für jede Untersuchungsnacht im Schlaflabor ist ein eigenes Polysomnographie-Protokoll beizufügen.

\section{Erläuterungen zu einzelnen Punkten:}

1. Patient (Initialen): [Wie in der pseudonymisierten Version des Arztbriefes]

Besonderheiten: [z. B. Medikation, körperl. Behinderung, psych. Erkrankung]

\section{Vor der Aufzeichnung}

Name Ableiter: [Name und Qualifikation (z. B. Krankenschwester) der Person, die die Ableitung durchgeführt hat und während der ganzen Nacht anwesend war]

Name verantwortlicher Arzt:. [Name des Arztes, der in der Anfangsphase der Einstellung (bei Überdrucktherapie) im Schlaflabor anwesend war und während der restlichen Nacht jederzeit rufbereit war]

Ableitprogramm (Konfiguration): [Ausdruck der Konfiguration (Kanäle, Filter, Verstärkung usw.) oder Beispielseite mit Beschriftung der Kanäle beilegen]

Zusatzkanäle: [z. B. Ösophagusdruck, Kapnographie, soweit nicht aus Ableitprogramm ersichtlich]

Modus: $\square$ CPAP $\square$ Bi-Level $\square$ APAP $\square$ anderer [z. B. Bi-level mit Zeittrigger (BIPAP-ST), ASV]

Weitere Parameter (Anfangseinstellungen) [z. B. Hintergrundfrequenz; Änderungen in der Notizenliste protokollieren!]

Maske: (Art, Typ, Größe): [Art z. B. Full Face, Nasenoliven, Individualmaske, Typ z. B. UltraMirage] 


\section{Durchführung}

Impedanzmessung: Alle Impedanzen unter KOhm? [im Schlaflabor verwendeten

Grenzwert einfügen]

Nicht korrigierbare Artefakte / Abweichungen: [z. B. „Schrittmacherartefakt"]

Beobachtungen während der Nacht (s. Notizenliste):

[Eine Liste der Beobachtungen und Interventionen während der Nacht ist beizufügen, etwa nach folgendem Schema:

\begin{tabular}{|l|l|}
\hline $\begin{array}{l}\text { Uhrzeit/ } \\
\text { Epoche }\end{array}$ & Beobachtung, Intervention \\
\hline & \\
\hline & \\
\hline
\end{tabular}

Einzutragen sind Änderungen der Therapieparameter mit Begründung, Medikamentengabe, auffälliges Verhalten oder Bewegungen des Patienten, Störungen, Besonderheiten]

\section{Auswertung}

Name Auswerter: [Name und Qualifikation (z. B. MTA) der Person, die die Ableitung visuell ausgewertet hat (Schlafstadienklassifikation, respiratorische Ereignisse usw.)

\section{Ergebnis der Auswertung}

[Ergebnisausdruck beifügen, wenn vorhanden. Aus diesem müssen die aufgeführten Werte hervorgehen. Weitere Eintragungen in diesem Abschnitt sind dann nicht erforderlich

\section{Latenzen:}

Schlaflatenz (ab Licht aus):

[Zeit bis zur ersten Epoche eines Schlafstadiums (nicht

Wach) ab Licht aus, falls eine andere Definition verwendet wird, bitte vermerken]

REM-Latenz (ab Schlafbeginn): (nicht Wach)]

[ab Schlafbeginn = erste Epoche eines Schlafstadiums

Prozentuale Anteile:

\begin{tabular}{|l|l|l|l|l|l|l|l|}
\hline Bezug & Wach & S1 / N1 & S2 / N2 & S3 / N3 & S4/-- & REM / R & MT / -- \\
\hline${ }^{*}$ & & & & & & \\
\hline
\end{tabular}

Hypopnoen [verwendete Definition angeben, z. B. AASM Standarddefinition, AASM optionale Definition]:

Bemerkungen: [z. B. zur Signalqualität, Ausfall von Kanälen, Besonderheiten]

\section{Befundung}

Befundung durch: [Name und Qualifikation (z. B. Arzt für Pneumologie, Somnologe) der Person, die den klinischen Befund für diese Ableitung erstellt hat.].

Befundbericht anhand der Originaldaten erstellt $\quad \square$ ja $\square$ nein

Befund: [Befundbericht beilegen, wenn der Befund nicht vollständig im Arztbrief oder einer Anlage dazu wiedergegeben ist. Weitere Eintragungen in diesem Abschnitt sind dann nicht erforderlich] 


\subsection{Ethikvotum}

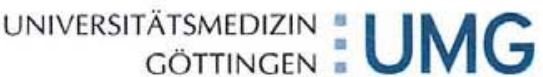

Ethikkommission der Med, Fakultat, Robert-Kocth-Stralbe 40, 37075 Göttingen

Frau

Ludmila Utte

Abt. Psychiatrie

- im Hause -
Medizinische Fakultät

Ethikkommission

Vorsitzender: Prof. Dr. Jürgen Brockmöller

Referentin

Regierungsrätin Doris Wettschereck

0551 / 39-8644 Telefon

37099 Göttingen Briefpost Robert-Koch-Straße 40, 37075 Göttingen

Adresse

0551 / 39-6629 Telefon 0551/39-9536 Fax

ethik@med.uni-goettingen.de E-Mail www.ethikkommission.med.uni-goettingen.de

vorab per E-Mail: ludmila-utte@gmx.net

25.07.2011 sc - gö Datum

Nachrichtlich an: Prof. Dr. rer. nat. Andrea Rodenbeck, E-Mail: arodenb@gwda.de

Antragsnummer: DOK_2_2011 (bitte stets angeben)

Studientitel: Qualitätssicherung in interdisziplinären Arbeitseinheiten am Beispiel Schlafmedizin

Antragsteller: Doktorandin: Ludmila Utte

Betreuer: Prof. Dr. rer. nat. Andrea Rodenbeck

Sehr geehrte Frau Utte, sehr geehrte Damen und Herren

wir bestätigen den Eingang Ihres Schreibens am 19.7.2011.

Die Ethik-Kommission stellt fest, dass es sich bei dem vorliegenden Antrag um kein durch eine medizinische Ethik-Kommission beratungspflichtiges medizinisches Forschungsvorhaben am Menschen handelt. Insofern besteht für dieses Projekt auch keine Verpflichtung der Beratung durch eine Ethik-Kommission. In meiner Funktion als Vorsitzender der Ethik-Kommission bestätige ich, dass aus meiner Sicht gegen das Projekt keine grundsätzlichen ethischen oder rechtlichen Bedenken bestehen.

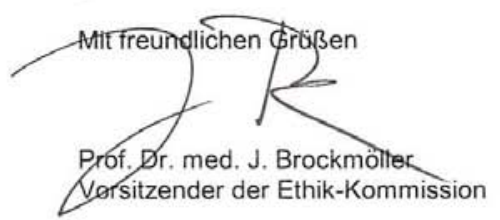

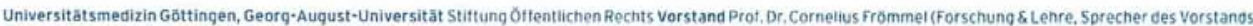
Prot. Dr. Wolltqanq Brück (komm. Krankenversorgung) Dipl. Kftr. (FH) Barbara Schulte (Wirtschaftsführung $\delta$ Administration) Sparkasse Gattingen (260500011) Kto:448 


\subsection{Erfassung der bewerteten Checkliste für die Schlaflabora- torien mit Weiterakkreditierung (verfahrensbezogen)}

Auswertung der bewerteten Checklisten. Aufgeführt sind die durch die Gutachter erfassten Items im Stichtagsverfahren (SV) und Randomisierten Verfahren (RV). Im SV wurden $(n=405)$ Patientenakten und im RV $(n=327)$ analysiert.

\begin{tabular}{|c|c|c|c|c|c|}
\hline Anamnese & Verfahren & vorhanden \% & nicht vorhanden (\%) & bereits extern durchgeführt (\%) & ohne Angabe (\%) \\
\hline \multirow[t]{2}{*}{ Allgemeine Anamnese } & SV & 77,3 & 13,3 & 8,6 & 0,8 \\
\hline & RV & 87,8 & 7,6 & 4 & 0,6 \\
\hline \multirow[t]{2}{*}{ Spez. Anamnese Nacht } & SV & 88,9 & 9,4 & 0 & 1,7 \\
\hline & RV & 93,3 & 5,5 & 0 & 1,2 \\
\hline \multirow[t]{2}{*}{ Spez. Anamnese Tag } & SV & 90,4 & 8,4 & 0 & 1,2 \\
\hline & RV & 95,1 & 3,7 & 0 & 1,2 \\
\hline \multirow[t]{2}{*}{ Organische Beschwerden } & SV & 82 & 14,1 & 1,7 & 2,2 \\
\hline & RV & 83,8 & 13,5 & 1,2 & 1,5 \\
\hline \multirow[t]{2}{*}{ Psychische Beschwerden } & SV & 62 & 32,1 & 1,5 & 4,4 \\
\hline & RV & 69,1 & 26 & 1,2 & 3,7 \\
\hline \multirow[t]{2}{*}{ Vegetative Beschwerden } & SV & 70,1 & 26,2 & 0,7 & 3 \\
\hline & RV & 70 & 25,7 & 1,5 & 2,8 \\
\hline \multirow[t]{2}{*}{ Psychosoziale Belastungen } & SV & 58,8 & 36 & 1,5 & 3,7 \\
\hline & RV & 61,2 & 31,5 & 2,8 & 4,5 \\
\hline \multirow[t]{2}{*}{$\begin{array}{l}\text { Risikofaktoren/ } \\
\text { Suchtanamnese }\end{array}$} & SV & 67,2 & 27,9 & 1,2 & 3,7 \\
\hline & RV & 70,6 & 22 & 1,9 & 5,5 \\
\hline \multirow[t]{2}{*}{$\begin{array}{c}\text { Bisherige Maßnahmen/DD } \\
\text { diskutiert }\end{array}$} & SV & 76,5 & 16,5 & 2,6 & 4,4 \\
\hline & RV & 72,5 & 19 & 3,9 & 4,6 \\
\hline
\end{tabular}


Anhang

\begin{tabular}{|c|c|c|c|c|c|c|c|}
\hline Diagnostik & Verfahren & $\begin{array}{c}\text { vorhanden } \\
\text { (\%) }\end{array}$ & $\begin{array}{c}\text { nicht vorhanden } \\
\text { (\%) }\end{array}$ & $\begin{array}{c}\text { bereits extern } \\
\text { durchgeführt (\%) }\end{array}$ & $\begin{array}{l}\text { Empfehlung } \\
\text { erfolgt (\%) }\end{array}$ & $\begin{array}{l}\text { Empfehlung } \\
\text { fehlt (\%) }\end{array}$ & $\begin{array}{l}\text { entfällt/ohne } \\
\text { Angabe (\%) }\end{array}$ \\
\hline \multirow[t]{2}{*}{$\begin{array}{c}\text { Subjektive } \\
\text { Befindlichkeit }\end{array}$} & sv & 79,9 & 14,3 & 1 & 0,2 & 0,2 & 4,4 \\
\hline & RV & 86,5 & 8 & 1,2 & 0,3 & 0 & 4 \\
\hline \multirow[t]{2}{*}{$\begin{array}{l}\text { Allg. körperlicher, } \\
\text { neurologischer, } \\
\text { psychiatrischer } \\
\text { Status }\end{array}$} & sV & 82,5 & 15,1 & 1,5 & 0,5 & 0,2 & 0,2 \\
\hline & $\mathrm{RV}$ & 84,4 & 12,2 & 1,5 & 0,9 & 0 & 1 \\
\hline \multirow[t]{2}{*}{ Größe, Gewicht, BMI } & sv & 85,2 & 11,4 & 1 & 0 & 0,7 & 1,7 \\
\hline & RV & 87,8 & 11,3 & 0 & 0 & 0 & 0,9 \\
\hline \multirow[t]{2}{*}{ Klinisches Labor } & sv & 50,9 & 27,7 & 8,9 & 0,7 & 2,2 & 9,6 \\
\hline & $\mathrm{RV}$ & 48,9 & 26 & 8,6 & 2,1 & 0 & 14,4 \\
\hline \multirow[t]{2}{*}{$\begin{array}{c}\text { Leistungs-, } \\
\text { Vigilanzuntersuchung }\end{array}$} & sv & 36 & 37,8 & 0,5 & 0,2 & 0,5 & 25 \\
\hline & $\mathrm{RV}$ & 32,1 & 40,1 & 0,3 & 0 & 0 & 27,5 \\
\hline \multirow{2}{*}{$\begin{array}{c}\text { Kardioresp. } \\
\text { Polysomnographie/Po } \\
\text { lygraphie/Monitoring }\end{array}$} & sv & 71,9 & 9,9 & 11,9 & 0,2 & 0,2 & 5,9 \\
\hline & $\mathrm{RV}$ & 73,1 & 7,6 & 16,8 & 0 & 0 & 2,5 \\
\hline \multirow[t]{2}{*}{$\begin{array}{l}\text { Komorbide Störungen } \\
\text { erkannt/diagnostiziert }\end{array}$} & sv & 65,2 & 10,1 & 8,9 & 2 & 1,2 & 12,6 \\
\hline & $\mathrm{RV}$ & 72,2 & 8,6 & 6,7 & 1,2 & 1,5 & 9,8 \\
\hline \multirow[t]{2}{*}{$\begin{array}{c}\text { Notwendige } \\
\text { Zusatzuntersuchung }\end{array}$} & sV & 38,5 & 14,1 & 5,7 & 7,4 & 2,5 & 31,8 \\
\hline & RV & 40,1 & 11,9 & 5,5 & 7,6 & 5,5 & 29,4 \\
\hline
\end{tabular}

\begin{tabular}{|c|c|c|c|c|c|}
\hline Messgrößen & Verfahren & vollständig (\%) & teilweise (\%) & fehlt (\%) & entfällt/ ohne Angabe (\%) \\
\hline \multirow[t]{2}{*}{ Globale Maße } & SV & 83 & 13,6 & 1,7 & 1,7 \\
\hline & RV & 85,3 & 10,1 & 3,1 & 1,5 \\
\hline \multirow[t]{2}{*}{ Latenzen } & SV & 84,4 & 7,4 & 5,7 & 2,5 \\
\hline & $\mathrm{RV}$ & 81,3 & 10,4 & 6,1 & 2,2 \\
\hline \multirow[t]{2}{*}{ Prozentuale Anteile } & SV & 88,6 & 5,7 & 4,7 & 1 \\
\hline & RV & 89,6 & 5,5 & 3,1 & 1,8 \\
\hline \multirow{2}{*}{$\begin{array}{l}\text { Atmung, Sättigung, } \\
\text { Schnarchen }\end{array}$} & SV & 88,1 & 10,9 & 0 & 1 \\
\hline & RV & 86,9 & 10,7 & 0,9 & 1,5 \\
\hline \multirow[t]{2}{*}{ Lage } & SV & 68,1 & 8,6 & 19 & \\
\hline & RV & 73,1 & 8,3 & 15,6 & 3 \\
\hline \multirow[t]{2}{*}{ Beinbewegung } & SV & 78,3 & 11,6 & 6,4 & 3,7 \\
\hline & RV & 82,3 & 8,3 & 5,5 & 3,9 \\
\hline \multirow[t]{2}{*}{ Arousal } & SV & 76,5 & 13,3 & 7,2 & 3 \\
\hline & RV & 82 & 10,7 & 4,9 & 2,4 \\
\hline
\end{tabular}


Anhang

\begin{tabular}{|c|c|c|c|c|c|c|}
\hline Therapie & Verfahren & $\begin{array}{c}\text { durchgeführt } \\
(\%)\end{array}$ & $\begin{array}{c}\text { terminiert } \\
(\%)\end{array}$ & $\begin{array}{c}\text { empfohlen } \\
(\%)\end{array}$ & $\begin{array}{c}\text { fehlt } \\
(\%)\end{array}$ & $\begin{array}{c}\text { entfällt/ohne } \\
\text { Angabe (\%) }\end{array}$ \\
\hline $\begin{array}{c}\text { Allgemeine } \\
\text { Behandlungsmaßnahmen }\end{array}$ & SV & 62 & 2 & 18 & 10,4 & 7,6 \\
\hline & RV & 60,9 & 0,6 & 20,5 & 8,3 & 9,7 \\
\hline $\begin{array}{c}\text { Psychologisch/ } \\
\text { Psychotherapeutisch }\end{array}$ & SV & 14,6 & 1,5 & 11,4 & 12,8 & 59,7 \\
\hline & RV & 7,6 & 0,6 & 11,7 & 11,3 & 68.8 \\
\hline $\begin{array}{c}\text { Medikamentöse } \\
\text { Behandlungsmaßnahmen }\end{array}$ & SV & 17 & 1 & 14,1 & 6,7 & 61,2 \\
\hline & RV & 10,7 & 0 & 8,9 & 7 & 73,4 \\
\hline Kontrolluntersuchung & SV & & 23,2 & 47,9 & 6,2 & 22,7 \\
\hline & RV & & 21,1 & 42,8 & 8,9 & 27,2 \\
\hline
\end{tabular}

\begin{tabular}{|c|c|c|c|c|c|c|}
\hline Entlassungsbericht & Verfahren & $\begin{array}{c}\text { angemessen } \\
(\%)\end{array}$ & $\begin{array}{c}\text { teilweise } \\
\text { angemessen } \\
(\%)\end{array}$ & $\begin{array}{c}\text { nicht } \\
\text { angemessen } \\
(\%) \\
\end{array}$ & fehlt (\%) & $\begin{array}{l}\text { entfällt/ohne } \\
\text { Angabe (\%) }\end{array}$ \\
\hline \multirow{2}{*}{$\begin{array}{c}\text { Verwendung der } \\
\text { Standardnomenklatur }\end{array}$} & SV & 77,8 & 18 & 2 & 1,7 & 0,5 \\
\hline & RV & 78,6 & 17,7 & 1,2 & 1,2 & 1,3 \\
\hline \multirow[t]{2}{*}{$\begin{array}{c}\text { Darstellung von } \\
\text { Problemen } \\
\end{array}$} & SV & 63 & 15,8 & 3,2 & 4,9 & 13,1 \\
\hline & RV & 63,3 & 18,3 & 4,3 & 5,5 & 8,6 \\
\hline \multirow[t]{2}{*}{$\begin{array}{c}\text { Stimmigkeit des } \\
\text { diagnostischen } \\
\text { Procedere } \\
\end{array}$} & SV & 73,1 & 20,2 & 4,2 & 1 & 1,5 \\
\hline & RV & 74,6 & 21,1 & 3,7 & 0 & 0,6 \\
\hline \multirow[t]{2}{*}{$\begin{array}{c}\text { Stimmigkeit des } \\
\text { therapeutischen } \\
\text { Procedere }\end{array}$} & SV & 69,4 & 21,2 & 4,2 & 1 & 4,2 \\
\hline & $\mathrm{RV}$ & 67,9 & 27,2 & 4 & 0 & 0,9 \\
\hline \multicolumn{7}{|l|}{$\begin{array}{c}\text { Zusammenfassende } \\
\text { Beurteilung }\end{array}$} \\
\hline \multirow[t]{2}{*}{ a)Schlafparameter } & SV & 77,5 & 13,1 & 4 & 2 & 3,4 \\
\hline & $\mathrm{RV}$ & 75,2 & 14,4 & 5,2 & 3,4 & 1,8 \\
\hline \multirow[t]{2}{*}{ b)Atmung } & SV & 85,4 & 10,6 & 1,5 & 1,5 & 1 \\
\hline & RV & 84,7 & 13,5 & 0,3 & 0,3 & 1,2 \\
\hline \multirow[t]{2}{*}{ c)Beinbewegung } & SV & 66,4 & 10,4 & 5,4 & 9,1 & 8,7 \\
\hline & RV & 68,8 & 10,1 & 5,2 & 5,8 & 10,1 \\
\hline
\end{tabular}




\subsection{Erfassung der bewerteten Checkliste für die Schlaflabora- torien ohne Weiterakkreditierung (nicht verfahrensbezogen)}

\begin{tabular}{|c|c|c|c|}
\hline Anamnese & vorhanden (\%) & nicht vorhanden (\%) & $\begin{array}{c}\text { bereits extern } \\
\text { durchgeführt (\%) }\end{array}$ \\
\hline Allgemeine Anamnese & 47,8 & 39,1 & 13,1 \\
\hline Spez. Anamnese Nacht & 61,8 & 38,2 & \\
\hline Spez. Anamnese Tag & 71 & 29 & 1,5 \\
\hline Organische Beschwerden & 39,7 & 58,8 & 1,5 \\
\hline Psychische Beschwerden & 23,9 & 74,6 & \\
\hline Vegetative Beschwerden & 30,9 & 69,1 & \\
\hline Psychosoziale Belastungen & 18,5 & 81,5 & 3 \\
\hline Risikofaktoren/Suchtanamnese & 32,3 & 67,7 & \\
\hline Bisherige Maßnahmen/DD diskutiert & 41,8 & 55,2 & \\
\hline
\end{tabular}

\begin{tabular}{|c|c|c|c|c|c|c|}
\hline Diagnostik & $\begin{array}{c}\text { vorhanden } \\
(\%)\end{array}$ & $\begin{array}{c}\text { nicht } \\
\text { vorhanden (\%) }\end{array}$ & $\begin{array}{c}\text { bereits extern } \\
\text { durchgeführt (\%) }\end{array}$ & $\begin{array}{c}\text { Empfehlung } \\
\text { ist erfolgt (\%) }\end{array}$ & $\begin{array}{c}\text { Empfehlung } \\
\text { fehlt (\%) }\end{array}$ & entfällt (\%) \\
\hline Subjektive Befindlichkeit & 53,7 & 41,8 & & & 4,5 \\
\hline $\begin{array}{c}\text { Allg. körperlicher, } \\
\text { neurologischer, } \\
\text { psychiatrischer Status }\end{array}$ & 49,3 & 49,3 & 1,4 & & & \\
\hline $\begin{array}{c}\text { Größe, Gewicht, BMI } \\
\text { Klinisches Labor }\end{array}$ & 62,3 & 36,2 & 1,5 & & 1,5 & 10,1 \\
\hline $\begin{array}{c}\text { Leistungs-, } \\
\text { Vigilanzuntersuchung }\end{array}$ & 17,4 & 56,5 & 7,2 & & 2,5 & 21,7 \\
\hline $\begin{array}{c}\text { Kardioresp. } \\
\text { Polysomnographie/Polygra } \\
\text { phie oder Monitoring }\end{array}$ & 59,4 & 23,2 & 14,5 & & & 2,9 \\
\hline $\begin{array}{c}\text { Komorbide Störungen } \\
\text { erkannt/diagnostiziert }\end{array}$ & 42,6 & 33,8 & 4,4 & & 3,4 & 16,2 \\
\hline $\begin{array}{c}\text { Notwendige } \\
\text { Zusatzuntersuchung }\end{array}$ & 13,2 & 44,1 & 4,4 & 5,9 & 7,4 & 25 \\
\hline
\end{tabular}

\begin{tabular}{|c|c|c|c|c|}
\hline Messgrößen & vollständig (\%) & teilweise (\%) & fehlt (\%) & entfällt (\%) \\
\hline Globale Maße & 69,7 & 19,7 & 10,6 & \\
\hline Latenzen & 67,7 & 12,3 & 20 & \\
\hline Prozentuale Anteile & 72,7 & 15,2 & 12,1 & \\
\hline $\begin{array}{c}\text { Atmung, Sättigung, } \\
\text { Schnarchen }\end{array}$ & 74,2 & 22,7 & 3,1 & \\
\hline Lage & 53 & 16,7 & 30,3 & 1,6 \\
\hline Beinbewegungen & 54,5 & 19,7 & 24,2 & \\
\hline Arousal & 59,1 & 18,2 & 22,7 & \\
\hline
\end{tabular}


Anhang

\begin{tabular}{|c|c|c|c|c|c|}
\hline Therapie & durchgeführt (\%) & terminiert (\%) & empfohlen (\%) & fehlt (\%) & entfällt (\%) \\
\hline $\begin{array}{c}\text { Allgemeine } \\
\text { Behandlungsmaßnahmen }\end{array}$ & 38,5 & 1,5 & 13,9 & 36,9 & 9,2 \\
\hline $\begin{array}{c}\text { Psychologisch/ } \\
\text { Psychotherapeutisch }\end{array}$ & 1,5 & & 6,2 & 24,6 & 67,7 \\
\hline $\begin{array}{c}\text { Medikamentöse } \\
\text { Behandlungsmaßnahmen }\end{array}$ & 10,6 & 9,1 & 12,1 & 68,2 \\
\hline Kontrolluntersuchung & & 10,5 & 59,1 & 15,2 & 15,2 \\
\hline
\end{tabular}

\begin{tabular}{|c|c|c|c|c|c|}
\hline Entlassungsbericht & $\begin{array}{c}\text { angemessen } \\
(\%)\end{array}$ & $\begin{array}{c}\text { teilweise } \\
\text { angemessen (\%) }\end{array}$ & $\begin{array}{c}\text { nicht angemessen } \\
(\%)\end{array}$ & fehlt (\%) & entfällt (\%) \\
\hline $\begin{array}{c}\text { Verwendung der } \\
\text { Standardnomenklatur }\end{array}$ & 61,8 & 25 & 8,8 & 4,4 & 3 \\
\hline $\begin{array}{c}\text { Stimmigkeit des } \\
\text { diagnostischen Procedere }\end{array}$ & 31,3 & 46,3 & 19,4 & & \\
\hline $\begin{array}{c}\text { Zusammenfassende } \\
\text { Beurteilung }\end{array}$ & 29,4 & 27,9 & 25 & 16,2 & 1,5 \\
\hline a)Schlafparameter & 41,2 & 35,3 & 14,7 & 7,3 & 1,5 \\
\hline b)Atmung & 27,9 & 22,1 & 23,5 & 23,5 & 3 \\
\hline c)Beinbewegung & 37,9 & 47 & 13,6 & 1,5 & \\
\hline $\begin{array}{c}\text { Stimmigkeit des } \\
\text { therapeutischen Procedere }\end{array}$ & 27,7 & 27,7 & 16,9 & 21,5 & 6,2 \\
\hline Darstellung von Problemen & & & & & 3 \\
\hline
\end{tabular}


